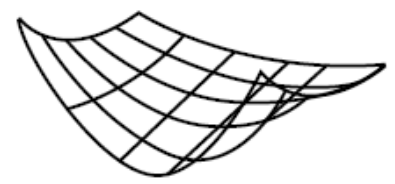

QUANTITATIVE FINANCE

RESEARCH CENTRE

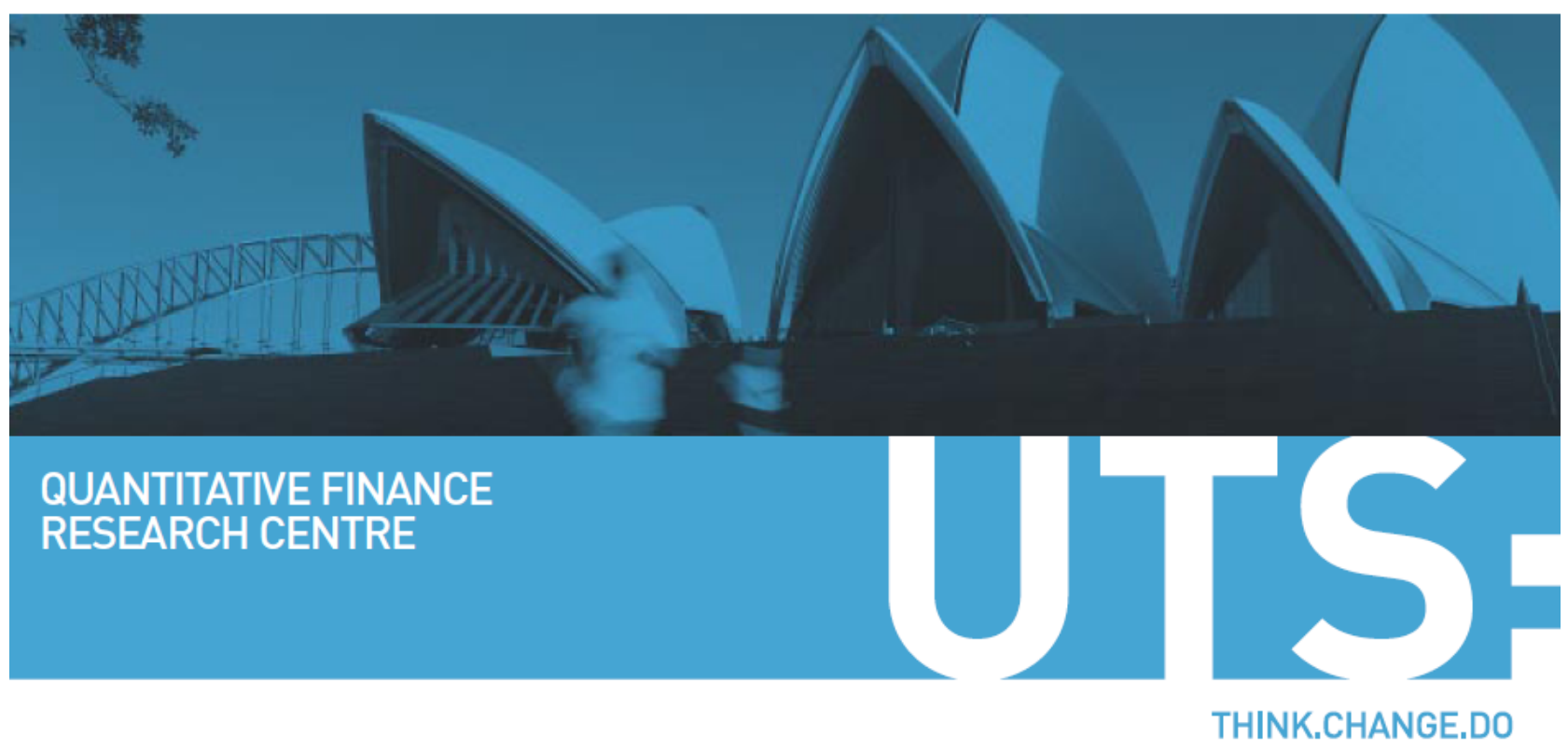

QuANTITATIVE FINANCE RESEARCH CENTRE

\title{
Two Stochastic Volatility Processes - American Option Pricing
}

Carl Chiarella and Jonathan Ziveyi 


\title{
Two Stochastic Volatility Processes - American Option Pricing
}

\author{
Carl Chiarella* and Jonathan Ziveyi ${ }^{\dagger}$
}

June 16, 2011

\begin{abstract}
In this paper we consider the pricing of an American call option whose underlying asset dynamics evolve under the influence of two independent stochastic volatility processes of the Heston (1993) type. We derive the associated partial differential equation (PDE) of the option price using hedging arguments and Ito's lemma. An integral expression for the general solution of the PDE is presented by using Duhamel's principle and this is expressed in terms of the joint transition density function for the driving stochastic processes. We solve the Kolmogorov PDE for the joint transition density function by first transforming it to a corresponding system of characteristic PDEs using a combination of Fourier and Laplace transforms. The characteristic PDE system is solved by using the method of characteristics. With the full price representation in place, numerical results are presented by first approximating the early exercise surface with a bivariate log linear function. We perform numerical comparisons with results generated by the method of lines algorithm and note that our approach is very competitive in terms of accuracy.
\end{abstract}

Keyword: American Options, Fourier Transform, Laplace Transform, Method of Characteristics.

JEL Classification: C61, D11

${ }^{*}$ Carl.Chiarella@uts.edu.au; School of Finance and Economics, University of Technology, Sydney, P.O. Box 123, Broadway, NSW 2007, Australia.

${ }^{\dagger}$ j.ziveyi@unsw.edu.au; Actuarial Studies, Australian School of Business, The University of New South Wales, Sydney, NSW 2052, Australia. 


\section{Introduction}

The standard option pricing framework, Black and Scholes (1973) has been premised on a number of restrictive assumptions, one of which is constant volatility of asset returns. The constant volatility assumption is based on the early perception that asset returns are characterized by the normal distribution.

Whilst the normality assumption of returns is a reasonable approach at long horizons, it is less satisfactory at horizons relevant to option pricing. Certainly empirical findings at shorter horizons reveal that asset returns are not normally distributed. Mandelbrot (1963), Officer (1972), Clark (1973), Blattberg and Gonedes (1974), Platen and Rendek (2008) among others postulate that the empirical distributions of asset returns are usually too peaked to be viewed as samples from Gaussian populations and suggests different types of distributions as possible candidates to model such changes. Many empirical studies also demonstrate that volatility of asset returns is not constant with Rosenberg (1972), Latané and Rendleman (1976) among others coming to the same conclusion through studies of implied volatility. Much work has followed with Scott (1987) also providing empirical evidence showing that volatility changes with time and that the changes are unpredictable. Scott notes that volatility has a tendency to revert to a long-run average. The mean-reverting feature has given birth to a range of research on European option pricing where the underlying asset is driven by stochastic mean reverting volatility processes. Scott (1987), Wiggins (1987), Hull and White (1987), Stein and Stein (1991), and Heston (1993), all consider European option pricing under stochastic volatility driven by various types of mean reverting processes.

Whilst most of the initial work has focused on European style options, not much has been done on pricing American option under stochastic volatility. Amongst the few papers on American option pricing, Touzi (1999) generalises the Black and Scholes (1973) model by allowing volatility to vary stochastically using optimal stopping theory of Karatzas (1988). Touzi describes the dependence of the early exercise boundary of the American put option on the volatility parameter and proves that such a boundary is a decreasing function of volatility implying that for a fixed underlying asset price, as the volatility increases, the early exercise boundary decreases. Clarke and Parrott (1999) develop an implicit finite-difference scheme for pricing American options written on underlying assets whose dynamics evolve under the influence of stochastic volatility. A multigrid algorithm is described for the fast iterative solution of the resulting discrete linear complementarity problems. Computational efficiency is also enhanced by a strike price related analytical transformation of the asset price and adaptive time-stepping.

Detemple and Tian (2002) provide analytical integral formulas for the early exercise boundary and the option price when the asset price follows a Constant Elasticity of Variance (CEV) process. The characteristic functions of the formulas are expressed in terms of $\mathcal{X}^{2}$ distribution functions. Tzavalis and Wang (2003) derive the integral representation of an American call option price when the volatility process evolves according to the square-root process proposed by Heston (1993). They derive the integral expressions again using optimal stopping 
theory along the lines of Karatzas (1988). By appealing to the empirical findings by Broadie, Detemple, Ghysels and Torres (2000) who show that the early exercise boundary when variance evolves stochastically is a log-linear function of both time and instantaneous variance, a Taylor series expansion is applied to the resulting early exercise surface around the long-run variance. The unknown functions resulting from the Taylor series expansion are then approximated by fitting Chebyshev polynomials. Ikonen and Toivanen (2004) formulate and solve the linear complementarity problem of the American call option under stochastic volatility using componentwise splitting methods. The resulting subproblems from componentwise splitting are solved by using standard partial differential equation methods.

Adolfsson, Chiarella, Ziogas and Ziveyi (2009) also derive the integral representation of the American call option under stochastic volatility by formulating the pricing PDE as an inhomogeneous problem and then using Duhamel's principle to represent the corresponding solution in terms of the joint transition density function. The joint density function solves the associated backward Kolmogorov PDE and a systematic approach for solving such a PDE is developed. A combination of Fourier and Laplace transforms is used to transform the homogeneous PDE for the density function to a characteristic PDE. The resulting system is then solved using ideas first presented by Feller (1951). The early exercise boundary is approximated by a log-linear function as proposed in Tzavalis and Wang (2003). Instead of using approximating polynomials as in Tzavalis and Wang (2003), Adolfsson et al. (2009) derive an explicit characteristic function for the early exercise premium component and then use numerical root finding techniques to find the unknown functions from the log-linear approximation.

There have also been attempts to generalise the Heston (1993) model to a multifactor specification for the volatility process in a single asset framework with da Fonseca, Grasselli and Tebaldi (2008) considering the pricing of European type options written on a single underlying asset whose dynamics evolve under the influence of the matrix Wishart volatility process. As demonstrated in da Fonseca et al. (2008) the main advantages of a multiple volatility system is that it calibrates short-term and long-term volatility levels better than a single process.

Motivated by the multifactor volatility feature, we seek to extend the American option pricing model of Adolfsson et al. (2009) to the multifactor stochastic volatility case. As a starting point we will assume that the underlying asset is driven by two stochastic variance processes of the Heston (1993) type. Whilst da Fonseca, Grasselli and Tebaldi (2005) and (2008) treat the two stochastic variance processes to be effective during different periods of the maturity domain, in this work we model the variance processes as independent risk factors influencing the dynamics of the underlying asset.

By first applying the Girsanov theorem for Wiener processes to the driving stochastic processes, we derive the corresponding pricing PDE using Ito's Lemma and some hedging arguments. The PDE is solved subject to initial and boundary conditions that specify the type of option under consideration. As is well known, the underlying asset of the American call option is bounded above by the early exercise boundary and below by zero. We convert 
the upper bound of the underlying asset to an unbounded domain by using the approach of Jamshidian (1992). The three stochastic processes; one for the underlying asset and the two variance processes can also be used to derive the corresponding PDE for their joint transition probability density function which satisfies a backward Kolmogorov PDE. Coupled with this and the unbounded PDE for the option price, we derive the general solution for the American option price by using Duhamel's principle. The only unknown term in the general solution is the transition density function which is the solution of the backward Kolmogorov PDE for the three driving processes.

In solving the Kolmogorov PDE, we first reduce it to a characteristic PDE by using a combination of Fourier and Laplace transforms. The resulting equation is then solved by the method of characteristics. Once the solution is found, we revert back to the original variables by applying the Fourier and Laplace inversion theorems. With the transition density in place, we can readily obtain the full integral representation of the American option price. As implied by Duhamel's principle, the American option price is the sum of two components namely the European and early exercise premium components. The European option component can be readily reduced to the Heston (1993) form by using similar techniques to those in Adolfsson et al. (2009). In dealing with the early exercise premium component, we extend the idea of Tzavalis and Wang (2003) and approximate the early exercise boundary as a bivariate loglinear function. This approximation allows us to reduce the integral dimensions of the early exercise premium by simplifying the integrals with respect to the two variance processes. The reduction of the dimensionality has the net effect of enhancing computational efficiency by reducing the computational time of the early exercise premium component.

This paper is organized as follows, we present the problem statement and the corresponding general solution of the American call option price in Section 2. We introduce key definitions of Fourier and Laplace transforms in Section 4. A Fourier transform is applied to the underlying asset variable in the PDE for the density function in Section 5 followed by application of a bivariate Laplace transform to the variance variables in Section 6. Application of the Laplace transform yields the PDE which we solve by the method of characteristics, details of which are given in Section 7. Once this PDE is solved the next step involves reverting back to the original underlying asset and variance variables. This is accomplished by applying Laplace and Fourier inversion theorems as detailed in Sections 8 and 9 respectively. The resulting function is the explicit representation of the transition density function. Section 10 nicely represents the integral form of the American call option price. An approximation of the early exercise boundary is presented in Section 11. Having found a representation of the American option price together with the early exercise boundary approximation, we then present details of how to implement the pricing relationship in Section 12. Numerical results are then presented in Section 13 followed by concluding remarks in Section 14. Lengthy derivations have been relegated to appendices. 


\section{Problem Statement}

In this paper we consider the evaluation of the American call option written on an underlying asset whose dynamics evolve under the influence of two stochastic variance processes of the Heston (1993) type. We represent the value of this option at the current time, $t$ as $V\left(t, S, v_{1}, v_{2}\right)$ where $S$ is the price of the underlying asset paying a continuously compounded dividend yield at a rate $q$ in a market offering a risk-free rate of interest denoted here as $r$, and $v_{1}$ and $v_{2}$ are the two variance processes driving $S$. Under the real world probability measure, $\mathbb{P}$, the underlying asset dynamics are governed by the stochastic differential equation (SDE) system

$$
\begin{aligned}
& d S=\mu S d t+\sqrt{v_{1}} S d Z_{1}+\sqrt{v_{2}} S d Z_{2}, \\
& d v_{1}=\kappa_{1}\left(\theta_{1}-v_{1}\right) d t+\sigma_{1} \sqrt{v_{1}} d Z_{3}, \\
& d v_{2}=\kappa_{2}\left(\theta_{2}-v_{2}\right) d t+\sigma_{2} \sqrt{v_{2}} d Z_{4}
\end{aligned}
$$

where $\mu$ is the instantaneous return per unit time of the underlying asset, $\theta_{1}$ and $\theta_{2}$ are the long-run means of $v_{1}$ and $v_{2}$ respectively, $\kappa_{1}$ and $\kappa_{2}$ are the speeds of mean-reversion, while $\sigma_{1}$ and $\sigma_{2}$ are the instantaneous volatilities of $v_{1}$ and $v_{2}$ per unit time respectively. The processes, $Z_{1}, Z_{2}, Z_{3}$ and $Z_{4}$ are correlated Wiener processes with a special correlation structure such that $\mathbb{E}^{\mathbb{P}}\left(d Z_{1} d Z_{3}\right)=\rho_{13} d t, \mathbb{E}^{\mathbb{P}}\left(d Z_{2} d Z_{4}\right)=\rho_{24} d t$ and all other correlations are zero.

We will need to apply Girsanov's Theorem for multiple Wiener processes. As this theorem is usually stated in terms of independent Wiener processes, it is convenient to transform the Wiener processes in the SDE system (2.1)-(2.3) to a corresponding system which is expressed in terms of independent Wiener processes whose increments we denote as $d W_{j}$ for $j=1, \cdots, 4$. This transformation is accomplished by performing the Cholesky decomposition such that

$$
\left[\begin{array}{l}
d Z_{1} \\
d Z_{2} \\
d Z_{3} \\
d Z_{4}
\end{array}\right]=\left[\begin{array}{cccc}
1 & 0 & 0 & 0 \\
0 & 1 & 0 & 0 \\
\rho_{13} & 0 & \sqrt{1-\rho_{13}^{2}} & 0 \\
0 & \rho_{24} & 0 & \sqrt{1-\rho_{24}^{2}}
\end{array}\right]\left[\begin{array}{c}
d W_{1} \\
d W_{2} \\
d W_{3} \\
d W_{4}
\end{array}\right]
$$

As highlighted in the correlation matrix above, we assume that correlation exists between the pairs, $\left(Z_{1}, Z_{3}\right)$ and $\left(Z_{2}, Z_{4}\right)$ such that all other correlation terms except $\rho_{13}$ and $\rho_{24}$ are zero. These assumptions about the correlation structure allow us to apply transform methods as we avoid the product term $\sqrt{v_{1}} \sqrt{v_{2}}$ which makes it impossible to apply the transform based methods that we propose. By incorporating the transformation (2.4) into equations (2.1)(2.3) we obtain the system of SDEs

$$
\begin{aligned}
& d S=\mu S d t+\sqrt{v_{1}} S d W_{1}+\sqrt{v_{2}} S d W_{2}, \\
& d v_{1}=\kappa_{1}\left(\theta_{1}-v_{1}\right) d t+\rho_{13} \sigma_{1} \sqrt{v_{1}} d W_{1}+\sqrt{1-\rho_{13}^{2}} \sigma_{1} \sqrt{v_{1}} d W_{3}, \\
& d v_{2}=\kappa_{2}\left(\theta_{2}-v_{2}\right) d t+\rho_{24} \sigma_{2} \sqrt{v_{2}} d W_{2}+\sqrt{1-\rho_{24}^{2}} \sigma_{2} \sqrt{v_{2}} d W_{4} .
\end{aligned}
$$


Using the approach of Feller (1951), for equations like (2.6) and (2.7) to be positive processes, the following conditions need to be satisfied:

$$
2 \kappa_{1} \theta_{1} \geq \sigma_{1}^{2} \quad \text { and } \quad 2 \kappa_{2} \theta_{2} \geq \sigma_{2}^{2}
$$

Cheang, Chiarella and Ziogas (2009) also show that in addition to the two conditions in (2.8) the following conditions:

$$
-1<\rho_{13}<\min \left(\frac{\kappa_{1}}{\sigma_{1}}, 1\right) \text { and }-1<\rho_{24}<\min \left(\frac{\kappa_{2}}{\sigma_{2}}, 1\right),
$$

need to be satisfied for the two variances to be finite. By following similar arguments to those in Cheang et al. (2009), it can be shown that the two conditions in equation (2.9) together with (2.8) also ensure that the solution of the underlying asset pricing process takes the form

$$
S_{t}=S_{0} \exp \left\{\mu t-\frac{1}{2} \int_{0}^{t} v_{1} d u-\frac{1}{2} \int_{0}^{t} v_{2} d u+\int_{0}^{t} \sqrt{v_{1}} d W_{1}+\int_{0}^{t} \sqrt{v_{2}} d W_{2}\right\}
$$

where

$$
\exp \left\{-\frac{1}{2} \int_{0}^{t} v_{1} d u-\frac{1}{2} \int_{0}^{t} v_{2} d u+\int_{0}^{t} \sqrt{v_{1}} d W_{1}+\int_{0}^{t} \sqrt{v_{2}} d W_{2}\right\}
$$

is a martingale under the real world probability measure, $\mathbb{P}$.

The system (2.5)-(2.7) contains four Wiener processes but only one traded asset $S$ as the two variance processes are non-tradable. This single asset is insufficient to hedge away these four risk factors when combined in a portfolio with an option dependent on the underlying asset, $S$. This situation leads to market incompleteness. In order to hedge away these risk sources, the market needs to be completed in some way. The process of completing the market is usually done by placing a sufficient number of options of different maturities in the hedging portfolio ${ }^{1}$.

The hedging technique usually results in the triplet of underlying processes , $\left(S, v_{1}, v_{2}\right)$ having different drift coefficients from those specified in the system, (2.5)-(2.7) thus resulting in different processes. We would however prefer to keep the original underlying asset price dynamics, a process achieved by switching from the real world probability measure, $\mathbb{P}$ to the risk-neutral probability measure, $\mathbb{Q}$. The change of measure is accomplished by making use of the Girsanov's Theorem for Wiener processes. Girsanov's Theorem ${ }^{2}$ uses the so-called Radon-Nikodym derivative, $\left(R_{N}\right)$ which takes the form (see for instance Cheang et al. (2009))

$$
R_{N}=\frac{d \mathbb{Q}}{d \mathbb{P}}=\exp \left\{-\frac{1}{2} \int_{0}^{t} \boldsymbol{\Lambda}_{u}^{T} \boldsymbol{\Sigma}^{-1} \boldsymbol{\Lambda}_{u} d u-\int_{0}^{t}\left(\boldsymbol{\Sigma}^{-1} \boldsymbol{\Lambda}_{u}\right)^{T} d \mathbf{W}\right\}
$$

where $\boldsymbol{\Sigma}$ is the correlation matrix in (2.4) and $\boldsymbol{\Lambda}_{t}$ is the vector of market prices of risk associated with the vector of Wiener processes, W. Market prices of risk associated with

\footnotetext{
${ }^{1}$ After applying these hedging arguments, it turns out that the resulting option pricing PDE is a function of two market prices of risk corresponding to the number of non-traded factors under consideration.

${ }^{2}$ For a detailed discussion see Harrison (1990).
} 
shocks on traded assets can be diversified away, however, for non-traded assets investors will always require a positive risk premium to compensate them for bearing such risk. Once the market prices of risk vector is specified, then by Girsanov's Theorem for Wiener processes there exist

$$
d \tilde{W}_{j}=\lambda_{j}(t) d t+d W_{j}
$$

where $\tilde{W}_{j}$, for $j=1, \cdots, 4$ are Wiener processes under the risk neutral measure $\mathbb{Q}$. From the vector, $\boldsymbol{\Lambda}_{t}$, we denote the constituent parameters as $\lambda_{1}(t)$ and $\lambda_{2}(t)$ to represent the market prices of risk associated with the Wiener instantaneous shocks, $d W_{1}$ and $d W_{2}$, on the underlying asset price dynamics, and $\lambda_{3}(t)$ and $\lambda_{4}(t)$ to be the market prices of risk associated with bearing the $d W_{3}$ and $d W_{4}$ risks on the non-traded variance factors, $v_{1}$ and $v_{2}$ respectively. As highlighted above, $\lambda_{3}(t)$ and $\lambda_{4}(t)$ cannot be diversified away as variance cannot be traded. Application of Girsanov's Theorem to the system (2.5)-(2.7) yields

$$
\begin{aligned}
& d S=(r-q) S d t+\sqrt{v_{1}} S d \tilde{W}_{1}+\sqrt{v_{2}} S d \tilde{W}_{2}, \\
& d v_{1}=\kappa_{1}\left(\theta_{1}-v_{1}\right) d t-\lambda_{3}(t) \sqrt{1-\rho_{13}^{2}} \sigma_{1} \sqrt{v_{1}} d t+\rho_{13} \sigma_{1} \sqrt{v_{1}} d \tilde{W}_{1}+\sqrt{1-\rho_{13}^{2}} \sigma_{1} \sqrt{v_{1}} d \tilde{W}_{3}, \\
& d v_{2}=\kappa_{2}\left(\theta_{2}-v_{2}\right) d t-\lambda_{4}(t) \sqrt{1-\rho_{24}^{2}} \sigma_{2} \sqrt{v_{2}} d t+\rho_{24} \sigma_{2} \sqrt{v_{2}} d \tilde{W}_{2}+\sqrt{1-\rho_{24}^{2}} \sigma_{2} \sqrt{v_{2}} d \tilde{W}_{4},
\end{aligned}
$$

where $r$ is the risk-free interest rate and $q$ is the continuously compounded dividend yield on the underlying asset, $S$. The key assumption we make on $\lambda_{3}(t)$ and $\lambda_{4}(t)$ is that both quantities are strictly positive to guarantee an investor a positive risk premium for holding the non-traded variance factors. In determining the market prices of the two variance risks, we use the same reasoning as in Heston (1993) with a slight modification such that

$$
\lambda_{3}(t)=\frac{\lambda_{1} \sqrt{v_{1}}}{\sigma_{1} \sqrt{1-\rho_{13}^{2}}}, \quad \text { and } \quad \lambda_{4}(t)=\frac{\lambda_{2} \sqrt{v_{2}}}{\sigma_{2} \sqrt{1-\rho_{24}^{2}}},
$$

where $\lambda_{1}$ and $\lambda_{2}$ are constants. This choice of market prices of risk

By substituting these into the system (2.14) we obtain

$$
\begin{aligned}
& d S=(r-q) S d t+\sqrt{v_{1}} S d \tilde{W}_{1}+\sqrt{v_{2}} S d \tilde{W}_{2}, \\
& d v_{1}=\left[\kappa_{1} \theta_{1}-\left(\kappa_{1}+\lambda_{1}\right) v_{1}\right] d t+\rho_{13} \sigma_{1} \sqrt{v_{1}} d \tilde{W}_{1}+\sqrt{1-\rho_{13}^{2}} \sigma_{1} \sqrt{v_{1}} d \tilde{W}_{3}, \\
& d v_{2}=\left[\kappa_{2} \theta_{2}-\left(\kappa_{2}+\lambda_{2}\right) v_{2}\right] d t+\rho_{24} \sigma_{2} \sqrt{v_{2}} d \tilde{W}_{2}+\sqrt{1-\rho_{24}^{2}} \sigma_{2} \sqrt{v_{2}} d \tilde{W}_{4} .
\end{aligned}
$$

The conditions in equations (2.8) and (2.9) also ensure that the explicit solution of the asset price process, (2.16) can be represented as

$$
S_{t}=S_{0} \exp \left\{(r-q) t-\frac{1}{2} \int_{0}^{t} v_{1} d u-\frac{1}{2} \int_{0}^{t} v_{2} d u+\int_{0}^{t} \sqrt{v_{1}} d \tilde{W}_{1}+\int_{0}^{t} \sqrt{v_{2}} d \tilde{W}_{2}\right\},
$$


where

$$
\exp \left\{-\frac{1}{2} \int_{0}^{t} v_{1} d u-\frac{1}{2} \int_{0}^{t} v_{2} d u+\int_{0}^{t} \sqrt{v_{1}} d \tilde{W}_{1}+\int_{0}^{t} \sqrt{v_{2}} d \tilde{W}_{2}\right\}
$$

is a positive martingale under the risk-neutral probability measure, $\mathbb{Q}$. Now with the system of equations (2.16)-(2.18), the next step involves the derivation of the corresponding American call option pricing PDE for the option written on the underlying asset, $S$. The pricing PDE can be shown to be ${ }^{3}$

$$
\frac{\partial V}{\partial \tau}\left(\tau, S, v_{1}, v_{2}\right)=\mathcal{L} V\left(\tau, S, v_{1}, v_{2}\right)-r V
$$

where

$$
\begin{aligned}
\mathcal{L}=( & r-q) S \frac{\partial}{\partial S}+\left[\kappa_{1}\left(\theta_{1}-v_{1}\right)-\lambda_{1} v_{1}\right] \frac{\partial}{\partial v_{1}}+\left[\kappa_{2}\left(\theta_{2}-v_{2}\right)-\lambda_{2} v_{2}\right] \frac{\partial}{\partial v_{2}} \\
+ & \frac{1}{2} v_{1} S^{2} \frac{\partial^{2}}{\partial S^{2}}+\frac{1}{2} \sigma_{1}^{2} v_{1} \frac{\partial^{2}}{\partial v_{1}^{2}}+\frac{1}{2} v_{2} S^{2} \frac{\partial^{2}}{\partial S^{2}}+\frac{1}{2} \sigma_{2}^{2} v_{2} \frac{\partial^{2}}{\partial v_{2}^{2}} \\
& +\rho_{13} \sigma_{1} v_{1} S \frac{\partial^{2}}{\partial S \partial v_{1}}+\rho_{14} \sigma_{2} v_{2} S \frac{\partial^{2}}{\partial S \partial v_{2}}
\end{aligned}
$$

Here, $\mathcal{L}$ is the Dynkin operator associated with the SDE system (2.16)-(2.18). The state variables are defined in the domains $0<v_{1}, v_{2}<\infty$ and $0 \leq S<b\left(\tau, v_{1}, v_{2}\right)$ where $S=$ $b\left(\tau, v_{1}, v_{2}\right)$, is the early exercise boundary of the American call option at time-to-maturity, $\tau$ when the instantaneous variances are $v_{1}$ and $v_{2}$ respectively. The PDE (2.21) is to be solved subject to the initial and boundary conditions

$$
\begin{aligned}
& V\left(0, S, v_{1}, v_{2}\right)=(S-K)^{+}, \quad 0<S<\infty, \\
& V\left(\tau, 0, v_{1}, v_{2}\right)=0, \quad \tau \geq 0, \\
& V\left(\tau, b\left(\tau, v_{1}, v_{2}\right), v_{1}, v_{2}\right)=b\left(\tau, v_{1}, v_{2}\right)-K, \quad \tau \geq 0, \\
& \quad \lim _{S \rightarrow b\left(\tau, v_{1}, v_{2}\right)} \frac{\partial V}{\partial S}\left(\tau, S, v_{1}, v_{2}\right)=1 \quad \tau \geq 0 .
\end{aligned}
$$

Condition (2.23) is the payoff of the option contract if it is held to maturity, while equation (2.24) is the absorbing state condition which ensures that the option ceases to exist once the underlying asset price hits zero. Equation (2.25) is the value matching condition which guarantees continuity of the option value function at the early exercise boundary, $b\left(\tau, v_{1}, v_{2}\right)$. Equation (2.26) is the smooth pasting condition which together with the value matching condition are imposed to eliminate arbitrage opportunities. Boundary conditions at $v_{1}=$ 0 and $v_{2}=0$ are found by extrapolation techniques when numerically implementing the resulting American call option pricing equation.

Also associated with the system of stochastic differential equations in (2.16)-(2.18) is the transition density function which we denote here as $G\left(\tau, S, v_{1}, v_{2} ; S_{0}, v_{1,0}, v_{2,0}\right)$. The transition

\footnotetext{
${ }^{3}$ Here $\tau=T-t$ is the time to maturity. Strictly speaking we should use different symbols to denote $V\left(T-\tau, S, v_{1}, v_{2}\right)$ and $V\left(\tau, S, v_{1}, v_{2}\right)$, but for convenience we use the same symbol.
} 
density function represents the transition probability of passage from $S, v_{1}, v_{2}$ at time-tomaturity $\tau$ to $S_{0}, v_{1,0}, v_{2,0}$ at maturity. It is well known that the transition density function satisfies the backward Kolmogorov PDE associated with the stochastic differential equations in the system (2.16)-(2.18) (see for example Chiarella (2010)). The Kolmogorov equation in the current situation can be shown to be of the form

$$
\frac{\partial G}{\partial \tau}=\mathcal{L} G
$$

where $0 \leq S<\infty$ and $0 \leq v_{1}, v_{2}<\infty$. Equation (2.27) is solved subject to the initial condition

$$
G\left(0, S, v_{1}, v_{2} ; S_{0}, v_{1,0}, v_{2,0}\right)=\delta\left(S-S_{0}\right) \delta\left(v_{1}-v_{1,0}\right) \delta\left(v_{2}-v_{2,0}\right),
$$

where $\delta(\cdot)$ is the Dirac delta function.

\section{Deriving the General Solution of the Pricing PDE}

As noted in the PDE (2.21), the underlying asset domain is bounded above by the early exercise boundary, $b\left(\tau, v_{1}, v_{2}\right)$. Jamshidian (1992) shows that one can consider an unbounded domain for the underlying asset by introducing an indicator function and transforming the PDE to

$$
\frac{\partial V}{\partial \tau}\left(\tau, S, v_{1}, v_{2}\right)=\mathcal{L} V\left(\tau, S, v_{1}, v_{2}\right)-r V+\mathbb{1}_{S \geq b\left(\tau, v_{1}, v_{2}\right)}(q S-r K)
$$

Here, $0 \leq S<\infty, 0<v_{1}, v_{2}<\infty$ and $\mathbb{1}_{S \geq b\left(\tau, v_{1}, v_{2}\right)}$ is an indicator function which is equal to one if $S \geq b\left(\tau, v_{1}, v_{2}\right)$ and zero otherwise. Now the PDE (3.1) is defined on an unbounded domain for the underlying asset.

As an initial step to solving the PDE (3.1), we switch to log asset variables by letting $S=e^{x}$ and setting

$$
\begin{aligned}
C\left(\tau, x, v_{1}, v_{2}\right) & \equiv V\left(\tau, e^{x}, v_{1}, v_{2}\right) \\
U\left(\tau, x, v_{1}, v_{2} ; x_{0}, v_{1,0}, v_{2,0}\right) & \equiv G\left(\tau, e^{x}, v_{1}, v_{2} ; e^{x_{0}}, v_{1,0}, v_{2,0}\right),
\end{aligned}
$$

to obtain

$$
\frac{\partial C}{\partial \tau}=\mathcal{M} C-r C+\mathbb{1}_{x \geq \ln b\left(\tau, v_{1}, v_{2}\right)}\left(q e^{x}-r K\right)
$$

where

$$
\begin{aligned}
\mathcal{M} & =\left(r-q-\frac{1}{2} v_{1}-\frac{1}{2} v_{2}\right) \frac{\partial}{\partial x}+\Phi_{1} \frac{\partial}{\partial v_{1}}-\beta_{1} v_{1} \frac{\partial}{\partial v_{1}}+\Phi_{2} \frac{\partial}{\partial v_{2}}-\beta_{2} v_{2} \frac{\partial}{\partial v_{2}}+\frac{1}{2} v_{1} \frac{\partial^{2}}{\partial x^{2}} \\
& +\frac{1}{2} v_{2} \frac{\partial^{2}}{\partial x^{2}}+\rho_{13} \sigma_{1} v_{1} \frac{\partial^{2}}{\partial x \partial v_{1}}+\rho_{14} \sigma_{2} v_{2} \frac{\partial^{2}}{\partial x \partial v_{2}}+\frac{1}{2} \sigma_{1}^{2} v_{1} \frac{\partial^{2}}{\partial v_{1}^{2}}+\frac{1}{2} \sigma_{2}^{2} v_{2} \frac{\partial^{2}}{\partial v_{2}^{2}}
\end{aligned}
$$




$$
\Phi_{1}=\kappa_{1} \theta_{1}, \quad \Phi_{2}=\kappa_{2} \theta_{2}, \quad \beta_{1}=\kappa_{1}+\lambda_{1} \quad \text { and } \quad \beta_{2}=\kappa_{2}+\lambda_{2}
$$

Equation (3.4) is solved subject to the initial condition

$$
C\left(0, x, v_{1}, v_{2}\right)=\left(e^{x}-K\right)^{+}, \quad-\infty<x<\infty .
$$

Likewise, the transition density PDE (2.27) is transformed to

$$
\frac{\partial U}{\partial \tau}=\mathcal{M} U
$$

Equation (3.8) is to be solved subject to the initial condition

$$
U\left(0, x, v_{1}, v_{2} ; x_{0}, v_{1,0}, v_{2,0}\right)=\delta\left(x-x_{0}\right) \delta\left(v_{1}-v_{1,0}\right) \delta\left(v_{2}-v_{2,0}\right)
$$

The inhomogeneous PDE (3.4) is in a form whose general solution can be represented by use of Duhamel's principle. ${ }^{4}$ We present the general solution of (3.4) in the proposition below.

Proposition 3.1 The solution of the American call option pricing PDE (3.4) can be represented as

$$
C\left(\tau, x, v_{1}, v_{2}\right)=C_{E}\left(\tau, x, v_{1}, v_{2}\right)+C_{P}\left(\tau, x, v_{1}, v_{2}\right)
$$

where

$$
C_{E}\left(\tau, x, v_{1}, v_{2}\right)=e^{-r \tau} \int_{0}^{\infty} \int_{0}^{\infty} \int_{-\infty}^{\infty}\left(e^{u}-K\right)^{+} U\left(\tau, x, v_{1}, v_{2} ; u, w_{1}, w_{2}\right) d u d w_{1} d w_{2}
$$

${ }^{4}$ Consider the one dimensional inhomogeneous parabolic PDE of the form

$$
\frac{\partial C}{\partial \tau}=\mathcal{L} C+f(\tau, x)
$$

where $\mathcal{L}$ is a parabolic partial differential operator and solved subject to the initial condition

$$
C(0, x)=\phi(x) .
$$

Let $U(\tau, x)$ be the transition probability density function which is the solution to

$$
\frac{\partial U}{\partial \tau}=\mathcal{L} U
$$

subject to the initial condition

$$
U(0, x)=\delta\left(x-x_{0}\right)
$$

then the solution of equation (3.10) can be represented as

$$
C(\tau, x)=\int_{-\infty}^{\infty} \phi(y) U(\tau, x-y) d y+\int_{0}^{\tau} \int_{-\infty}^{\infty} f(\xi, y) U(\tau-\xi, x-y) d y d \xi .
$$

More details about Duhamel's principle can be found in Logan (2004). 
and

$$
\begin{aligned}
C_{P}\left(\tau, x, v_{1}, v_{2}\right)= & \int_{0}^{\tau} e^{-r(\tau-\xi)} \int_{0}^{\infty} \int_{0}^{\infty} \int_{\ln b\left(\xi, w_{1}, w_{2}\right)}^{\infty}\left(q e^{u}-r K\right) \\
& \times U\left(\tau-\xi, x, v_{1}, v_{2} ; u, w_{1}, w_{2}\right) d u d w_{1} d w_{2} d \xi
\end{aligned}
$$

Proof: Refer to Appendix 1.

The first component of equation (3.14) is the European option component whilst the second component is the early exercise premium. For us to operationalise the representation of equation (3.14), we need an explicit form of the density function, $U\left(\tau, x, v_{1}, v_{2}\right)$ which is the solution of the PDE in equation (3.8). In order to solve equation (3.8), we first reduce it to a corresponding system of characteristic PDEs which can then be readily solved using a vast array of methods for tackling such problems. In this paper, we will apply a combination of Fourier and Laplace transforms to this PDE resulting in a system of characteristic PDEs. We will apply Fourier transforms to the underlying log asset variable as its domain matches that of the transform. A bivariate Laplace transform will then be applied to the stochastic variance variables. We start by giving a brief review of the transform methods before applying them to the PDE (3.8).

\section{Fourier and Laplace Transforms}

Definition 4.1 The Fourier transform of the function $U\left(\tau, x, v_{1}, v_{2}\right)$ with respect to $x$ is defined as,

$$
\mathcal{F}\left\{U\left(\tau, x, v_{1}, v_{2}\right)\right\}=\int_{-\infty}^{\infty} e^{i \eta x} U\left(\tau, x, v_{1}, v_{2}\right) d x:=\hat{U}\left(\tau, \eta, v_{1}, v_{2}\right)
$$

where $i$ is the complex number.

Definition 4.2 The inverse of the Fourier transform of $\hat{U}\left(\tau, \eta, v_{1}, v_{2}\right)$ is represented as,

$$
\mathcal{F}^{-1}\left\{\hat{U}\left(\tau, \eta, v_{1}, v_{2}\right)\right\}=\frac{1}{2 \pi} \int_{-\infty}^{\infty} e^{-i \eta x} \hat{U}\left(\tau, \eta, v_{1}, v_{2}\right) d \eta:=U\left(\tau, x, v_{1}, v_{2}\right)
$$

Definition 4.3 The bivariate Laplace transform of the function $\hat{U}\left(\tau, \eta, v_{1}, v_{2}\right)$ with respect to $v_{1}$ and $v_{2}$ is defined as,

$$
\mathcal{L}\left\{\hat{U}\left(\tau, \eta, v_{1}, v_{2}\right)\right\}=\int_{0}^{\infty} \int_{0}^{\infty} e^{-s_{1} v_{1}-s_{2} v_{2}} \hat{U}\left(\tau, \eta, v_{1}, v_{2}\right) d v_{1} d v_{2}:=\tilde{U}\left(\tau, \eta, s_{1}, s_{2}\right),
$$

where $s_{1}$ and $s_{2}$ are complex variables whenever the improper integral exists.

Definition 4.4 The bivariate inverse Laplace transform ${ }^{5}$ of the function $\tilde{U}\left(\tau, \eta, s_{1}, s_{2}\right)$ with

\footnotetext{
${ }^{5}$ In this paper we will not directly use this inverse Laplace transform definition as we will make use of those tabulated in Abramowitz and Stegun (1964).
} 
respect to $s_{1}$ and $s_{2}$ is defined as,

$\mathcal{L}^{-1}\left\{\tilde{U}\left(\tau, \eta, s_{1}, s_{2}\right)\right\}=\frac{1}{(2 \pi i)^{2}} \int_{\gamma_{2}-i \infty}^{\gamma_{2}+i \infty} \int_{\gamma_{1}-i \infty}^{\gamma_{1}+i \infty} e^{s_{1} v_{1}+s_{2} v_{2}} \tilde{U}\left(\tau, \eta, s_{1}, s_{2}\right) d s_{1} d s_{2}:=\hat{U}\left(\tau, \eta, v_{1}, v_{2}\right)$

where the integration is done along the lines, $\operatorname{Re}\left(s_{1}\right)=\gamma_{1}$ and $\operatorname{Re}\left(s_{2}\right)=\gamma_{2}$ in the complex hyperplane such that $\gamma_{1}$ and $\gamma_{2}$ are greater than the real part of all singularities of $\tilde{U}\left(\tau, \eta, s_{1}, s_{2}\right)$.

We will make the following assumptions about this density function:

$$
\begin{aligned}
& \lim _{x \rightarrow \pm \infty} U\left(\tau, x, v_{1}, v_{2}\right)=\lim _{x \rightarrow \pm \infty} \frac{\partial U}{\partial x}=0, \\
& \lim _{v_{1}, v_{2} \rightarrow \infty} U\left(\tau, x, v_{1}, v_{2}\right)=\lim _{v_{1} \rightarrow \infty} \frac{\partial U}{\partial v_{1}}=\lim _{v_{2} \rightarrow \infty} \frac{\partial U}{\partial v_{2}}=0 \\
& \lim _{v_{1}, v_{2} \rightarrow 0} U\left(\tau, x, v_{1}, v_{2}\right)=0 .
\end{aligned}
$$

Such assumptions are made since by their nature, transition density functions and their derivatives converge to zero as their underlying processes assume larger values.

\section{$5 \quad$ Applying Fourier Transforms}

We first apply the Fourier transform to the log asset price variable in the PDE (3.8).

Proposition 5.1 The Fourier transform, $\hat{U}\left(\tau, \eta, v_{1}, v_{2}\right)$ of the function $U\left(\tau, x, v_{1}, v_{2}\right)$ which is the solution of the homogeneous PDE (3.8) satisfies the PDE

$$
\begin{aligned}
\frac{\partial \hat{U}}{\partial \tau}= & -i \eta(r-q) \hat{U}+\frac{1}{2} \Lambda(\eta) v_{1} \hat{U}+\frac{1}{2} \Lambda(\eta) v_{2} \hat{U}+\Phi_{1} \frac{\partial \hat{U}}{\partial v_{1}}+\Phi_{2} \frac{\partial \hat{U}}{\partial v_{2}} \\
& -\Theta_{1} v_{1} \frac{\partial \hat{U}}{\partial v_{1}}-\Theta_{2} v_{2} \frac{\partial \hat{U}}{\partial v_{2}}+\frac{1}{2} \sigma_{1}^{2} v_{1} \frac{\partial^{2} \hat{U}}{\partial v_{1}^{2}}+\frac{1}{2} \sigma_{2}^{2} v_{2} \frac{\partial^{2} \hat{U}}{\partial v_{2}^{2}}
\end{aligned}
$$

where

$$
\Theta_{1}=\Theta_{1}(\eta) \equiv \beta_{1}+i \eta \rho_{13} \sigma_{1}, \quad \Theta_{2}=\Theta_{2}(\eta) \equiv \beta_{2}+i \eta \rho_{24} \sigma_{2}, \quad \text { and } \quad \Lambda(\eta)=i \eta-\eta^{2} .
$$

Equation (5.1) is to be solved subject to the initial condition

$$
\hat{U}\left(0, \eta, v_{1}, v_{2}\right)=e^{i \eta x_{0}} \delta\left(v_{1}-v_{1,0}\right) \delta\left(v_{2}-v_{2,0}\right) .
$$

Proof: Refer to Appendix 2. 


\section{Applying Laplace Transforms}

We have applied the Fourier transform to the log asset price variable. To successfully solve the resulting PDE (5.1), we apply a bivariate Laplace transform to the variance variables and this is accomplished by the proposition below.

Proposition 6.1 By applying Definition 4.3 to the PDE (5.1) the Laplace transform, $\tilde{U}\left(\tau, \eta, s_{1}, s_{2}\right)$ is found to satisfy the first order PDE

$$
\begin{aligned}
\frac{\partial \tilde{U}}{\partial \tau} & +\left\{\frac{1}{2} \sigma_{1}^{2} s_{1}^{2}-\Theta_{1} s_{1}+\frac{1}{2} \Lambda(\eta)\right\} \frac{\partial \tilde{U}}{\partial s_{1}}+\left\{\frac{1}{2} \sigma_{2}^{2} s_{2}^{2}-\Theta_{2} s_{2}+\frac{1}{2} \Lambda(\eta)\right\} \frac{\partial \tilde{U}}{\partial s_{2}} \\
& =\left\{\left(\Phi_{1}-\sigma_{1}^{2}\right) s_{1}+\left(\Phi_{2}-\sigma_{2}^{2}\right) s_{2}-i \eta(r-q)+\Theta_{1}+\Theta_{2}\right\} \tilde{U}+f_{1}\left(\tau, s_{2}\right)+f_{2}\left(\tau, s_{1}\right) .
\end{aligned}
$$

Equation (6.1) is to be solved subject to the initial condition

$$
\tilde{U}\left(0, \eta, s_{1}, s_{2}\right)=e^{i \eta x_{0}-s_{1} v_{1,0}-s_{2} v_{2,0}} .
$$

The two functions, $f_{1}$ and $f_{2}$ are given by

$$
f_{1}\left(\tau, s_{2}\right)=\left(\frac{1}{2} \sigma_{1}^{2}-\Phi_{1}\right) \tilde{U}\left(\tau, \eta, 0, s_{2}\right), \quad \text { and } \quad f_{2}\left(\tau, s_{1}\right)=\left(\frac{1}{2} \sigma_{2}^{2}-\Phi_{2}\right) \tilde{U}\left(\tau, \eta, s_{1}, 0\right)
$$

and are determined such that

$$
\lim _{s_{1} \rightarrow \infty} \tilde{U}\left(\tau, \eta, s_{1}, s_{2}\right)=0, \quad \text { and } \quad \lim _{s_{2} \rightarrow \infty} \tilde{U}\left(\tau, \eta, s_{1}, s_{2}\right)=0
$$

respectively.

Proof: Refer to Appendix 3.

\section{Solution of the Characteristic Equation}

Equation (6.1) is a first order PDE known as a characteristic equation. We solve this equation using the method of characteristics. Similar techniques have been successfully used in Feller (1951) and Adolfsson et al. (2009) to solve PDEs like equation (6.1). Cheang et al. (2009) use similar techniques when solving the American call option where the underlying asset is being driven by both stochastic volatility and jumps. We present the solution of this PDE in the proposition below.

Proposition 7.1 The solution of equation (6.1) subject to the initial and boundary conditions 
in equations (6.2) and (6.3) can be represented as

$$
\begin{aligned}
\tilde{U}\left(\tau, \eta, s_{1}, s_{2}\right)=\left(\frac{2 \Omega_{1}}{\left(\sigma_{1}^{2} s_{1}-\Theta_{1}+\Omega_{1}\right)\left(e^{\Omega_{1} \tau}-1\right)+2 \Omega_{1}}\right)^{2-\frac{2 \Phi_{1}}{\sigma_{1}^{2}}}\left(\frac{2 \Omega_{2}}{\left(\sigma_{2}^{2} s_{2}-\Theta_{2}+\Omega_{2}\right)\left(e^{\Omega_{2} \tau}-1\right)+2 \Omega_{2}}\right)^{2-\frac{2 \Phi_{2}}{\sigma_{2}^{2}}} \\
\times \exp \left\{-\left(\frac{\Theta_{1}-\Omega_{1}}{\sigma_{1}^{2}}\right) v_{1,0}-\left(\frac{\Theta_{2}-\Omega_{2}}{\sigma_{2}^{2}}\right) v_{2,0}+i \eta x_{0}\right\} \\
\times \exp \left\{\left[\frac{\left(\Phi_{1}-\sigma_{1}^{2}\right)\left(\Theta_{1}-\Omega_{1}\right)}{\sigma_{1}^{2}}+\frac{\left(\Phi_{2}-\sigma_{2}^{2}\right)\left(\Theta_{2}-\Omega_{2}\right)}{\sigma_{2}^{2}}-i \eta(r-q)+\Theta_{1}+\Theta_{2}\right] \tau\right\} \\
\times \exp \left\{\frac{-2 \Omega_{1} v_{1,0}\left(\sigma_{1}^{2} s_{1}-\Theta_{1}+\Omega_{1}\right) e^{\Omega_{1} \tau}}{\sigma_{1}^{2}\left[\left(\sigma_{1}^{2} s_{1}-\Theta_{1}+\Omega_{1}\right)\left(e^{\Omega_{1} \tau}-1\right)+2 \Omega_{1}\right]}\right\} \exp \left\{\frac{-2 \Omega_{2} v_{2,0}\left(\sigma_{2}^{2} s_{2}-\Theta_{2}+\Omega_{2}\right) e^{\Omega_{2} \tau}}{\sigma_{2}^{2}\left[\left(\sigma_{2}^{2} s_{2}-\Theta_{2}+\Omega_{2}\right)\left(e^{\Omega_{2} \tau}-1\right)+2 \Omega_{2}\right]}\right\} \\
\times\left[\Gamma\left(\frac{2 \Phi_{1}}{\sigma_{1}^{2}}-1 ; \frac{2 \Omega_{1} v_{1,0} e^{\Omega_{1} \tau}}{\sigma_{1}^{2}\left(e^{\Omega_{1} \tau}-1\right)} \times \frac{2 \Omega_{1}}{\left(\sigma_{1}^{2} s_{1}-\Theta_{1}+\Omega_{1}\right)\left(e^{\Omega_{1} \tau}-1\right)+2 \Omega_{1}}\right)\right. \\
\left.+\Gamma\left(\frac{2 \Phi_{2}}{\sigma_{2}^{2}}-1 ; \frac{2 \Omega_{2} v_{2,0} e^{\Omega_{2} \tau}}{\sigma_{2}^{2}\left(e^{\Omega_{2} \tau}-1\right)} \times \frac{2 \Omega_{2}}{\left(\sigma_{2}^{2} s_{2}-\Theta_{2}+\Omega_{2}\right)\left(e^{\Omega_{2} \tau}-1\right)+2 \Omega_{2}}\right)-1\right]
\end{aligned}
$$

where

$$
\Omega_{1}=\sqrt{\Theta_{1}^{2}-\Lambda(\eta) \sigma_{1}^{2}} \text { and } \Omega_{2}=\sqrt{\Theta_{2}^{2}-\Lambda(\eta) \sigma_{2}^{2}}
$$

The function $\Gamma(n ; z)$ is the incomplete gamma function defined as

$$
\Gamma(n ; z)=\frac{1}{\Gamma(n)} \int_{0}^{z} e^{-\xi} \xi^{n-1} d \xi
$$

and

$$
\Gamma(n)=\int_{0}^{\infty} e^{-\xi} \xi^{n-1} d \xi
$$

Proof: Refer to Appendix 4.

\section{Inverting the Laplace Transform}

Now that we have solved equation (6.1), the next step is to recover the original function, $U\left(\tau, x, v_{1}, v_{2}\right)$ which is expressed in terms of the original state variables. This process is accomplished by applying the Laplace and Fourier inversion theorems respectively. In Proposition 8.1 below, we will first present the inverse Laplace transform of the function given in Proposition 7.1. 
Proposition 8.1 The inverse Laplace transform of $\tilde{U}\left(\tau, \eta, s_{1}, s_{2}\right)$ in equation (7.1) is

$$
\begin{aligned}
& \hat{U}\left(\tau, \eta, v_{1}, v_{2}\right)=\exp \left\{\left(\frac{\Theta_{1}-\Omega_{1}}{\sigma_{1}^{2}}\right)\left(v_{1}-v_{1,0}+\Phi_{1} \tau\right)+\left(\frac{\Theta_{2}-\Omega_{2}}{\sigma_{2}^{2}}\right)\left(v_{2}-v_{2,0}+\Phi_{2} \tau\right)\right\} \\
& \times \exp \left\{-\left(\frac{2 \Omega_{1}}{\sigma_{1}^{2}\left(e^{\Omega_{1} \tau}-1\right)}\right)\left(v_{1,0} e^{\Omega_{1} \tau}+v_{1}\right)-\left(\frac{2 \Omega_{2}}{\sigma_{2}^{2}\left(e^{\Omega_{2} \tau}-1\right)}\right)\left(v_{2,0} e^{\Omega_{2} \tau}+v_{2}\right)\right\} \\
& \times e^{i \eta x_{0}-i \eta(r-q) \tau} \frac{2 \Omega_{1} e^{\Omega_{1} \tau}}{\sigma_{1}^{2}\left(e^{\Omega_{1} \tau}-1\right)} \frac{2 \Omega_{2} e^{\Omega_{2} \tau}}{\sigma_{2}^{2}\left(e^{\Omega_{2} \tau}-1\right)}\left(\frac{v_{1,0} e^{\Omega_{1} \tau}}{v_{1}}\right)^{\frac{\Phi_{1}}{\sigma_{1}^{2}-\frac{1}{2}}}\left(\frac{v_{2,0} e^{\Omega_{2} \tau}}{v_{2}}\right)^{\frac{\Phi_{2}}{\sigma_{2}^{2}}-\frac{1}{2}} \\
& \quad \times I_{\frac{2 \Phi_{1}}{\sigma_{1}^{2}}-1}\left(\frac{4 \Omega_{1}}{\sigma_{1}^{2}\left(e^{\Omega_{1} \tau}-1\right)}\left(v_{1} v_{1,0} e^{\Omega_{1} \tau}\right)^{\frac{1}{2}}\right) I_{\frac{2 \Phi_{2}}{\sigma_{2}^{2}}-1}\left(\frac{4 \Omega_{2}}{\sigma_{2}^{2}\left(e^{\Omega_{2} \tau}-1\right)}\left(v_{2} v_{2,0} e^{\Omega_{2} \tau}\right)^{\frac{1}{2}}\right),
\end{aligned}
$$

where $I_{k}(z)$ is the modified Bessel function of the first kind defined as

$$
I_{k}(z)=\sum_{n=0}^{\infty} \frac{\left(\frac{z}{2}\right)^{2 n+k}}{\Gamma(n+k+1) n !} .
$$

Proof: Refer to Appendix 5.

\section{Inverting the Fourier Transform}

The next task is to find the inverse Fourier transform of equation (8.1), this is accomplished by applying Definition 4.2 to Proposition 8.1 and we present the result in the proposition below.

Proposition 9.1 The inverse Fourier transform, $U\left(\tau, x, v_{1}, v_{2} ; x_{0}, v_{1,0}, v_{2,0}\right)$ of equation (8.1) can be expressed as

$$
U\left(\tau, x, v_{1}, v_{2} ; x_{0}, v_{1,0}, v_{2,0}\right)=\frac{1}{2 \pi} \int_{-\infty}^{\infty} e^{i \eta x_{0}} H\left(\tau, x, v_{1}, v_{2} ;-\eta, v_{1,0}, v_{2,0}\right) d \eta
$$

where

$$
\begin{aligned}
& H\left(\tau, x, v_{1}, v_{2} ; \eta, v_{1,0}, v_{2,0}\right)=\exp \left\{\left(\frac{\Theta_{1}-\Omega_{1}}{\sigma_{1}^{2}}\right)\left(v_{1}-v_{1,0}+\Phi_{1} \tau\right)+\left(\frac{\Theta_{2}-\Omega_{2}}{\sigma_{2}^{2}}\right)\left(v_{2}-v_{2,0}+\Phi_{2} \tau\right)\right\} \\
& \times \exp \left\{-\left(\frac{2 \Omega_{1}}{\sigma_{1}^{2}\left(e^{\Omega_{1} \tau}-1\right)}\right)\left(v_{1,0} e^{\Omega_{1} \tau}+v_{1}\right)-\left(\frac{2 \Omega_{2}}{\sigma_{2}^{2}\left(e^{\Omega_{2} \tau}-1\right)}\right)\left(v_{2,0} e^{\Omega_{2} \tau}+v_{2}\right)\right\} \\
& \quad \times e^{i \eta x+i \eta(r-q) \tau} \frac{2 \Omega_{1} e^{\Omega_{1} \tau}}{\sigma_{1}^{2}\left(e^{\Omega_{1} \tau}-1\right)} \frac{2 \Omega_{2} e^{\Omega_{2} \tau}}{\sigma_{2}^{2}\left(e^{\Omega_{2} \tau}-1\right)}\left(\frac{v_{1,0} e^{\Omega_{1} \tau}}{v_{1}}\right)^{\frac{\Phi_{1}}{\sigma_{1}^{2}-\frac{1}{2}}}\left(\frac{v_{2,0} e^{\Omega_{2} \tau}}{v_{2}}\right)^{\frac{\Phi_{2}}{\sigma_{2}^{2}}-\frac{1}{2}} \\
& \quad \times I_{\frac{2 \Phi_{1}}{\sigma_{1}^{2}}-1}\left(\frac{4 \Omega_{1}}{\sigma_{1}^{2}\left(e^{\Omega_{1} \tau}-1\right)}\left(v_{1} v_{1,0} e^{\Omega_{1} \tau}\right)^{\frac{1}{2}}\right) I_{\frac{2 \Phi_{2}}{\sigma_{2}^{2}}-1}\left(\frac{4 \Omega_{2}}{\sigma_{2}^{2}\left(e^{\Omega_{2} \tau}-1\right)}\left(v_{2} v_{2,0} e^{\Omega_{2} \tau}\right)^{\frac{1}{2}}\right) .
\end{aligned}
$$

Proof: Refer to Appendix 6. 
Now that we have managed to obtain $U\left(\tau, x, v_{1}, v_{2}\right)$, we revert back to the original density function $G\left(\tau, S, v_{1}, v_{2}\right)$ which is expressed in terms of the underlying asset variable, $S$.

Proposition 9.2 The transition density function expressed in terms of the original state variables can be represented as

$$
G\left(\tau, S, v_{1}, v_{2} ; S_{0}, v_{1,0}, v_{2,0}\right)=\frac{1}{2 \pi} \int_{-\infty}^{\infty} e^{i \eta \ln S_{0}} H\left(\tau, \ln S, v_{1}, v_{2} ;-\eta, v_{1,0}, v_{2,0}\right) d \eta
$$

Proof: Recall that $S \equiv e^{x}$ and $U\left(\tau, x, v_{1}, v_{2} ; x_{0}, v_{1,0}, v_{2,0}\right) \equiv G\left(\tau, e^{x}, v_{1}, v_{2} ; e^{x_{0}}, v_{1,0}, v_{2,0}\right)$. Substituting these into equation (9.1) we obtain the result in the above proposition.

Having found the explicit form of the trivariate transition density function, we can now obtain the full representation of the American call option presented in Proposition 3.1. As demonstrated in that proposition, the American option price is expressed in terms of the unknown early exercise boundary $S=b\left(\tau, v_{1}, v_{2}\right)$. We use the value matching condition to find the integral equation that determines this function, details of which are given in the next section.

\section{The American Option Price}

As stated in the previous section, given the explicit transition probability density function in Proposition 9.2, we can derive the simplified version of the American call option written on the underlying asset, $S$ whose dynamics evolve according to the SDE system (2.16)-(2.18). By using the relationship $C\left(\tau, x, v_{1}, v_{2}\right) \equiv V\left(\tau, e^{x}, v_{1}, v_{2}\right)$, the value of the American call option can be represented as

$$
V\left(\tau, S, v_{1}, v_{2}\right)=V_{E}\left(\tau, S, v_{1}, v_{2}\right)+V_{P}\left(\tau, S, v_{1}, v_{2}\right)
$$

The two terms on the RHS of equation (10.1) are presented in the two propositions below. As pointed out earlier, the first component on the RHS of equation (10.1) is the European option component whilst the second is the early exercise premium.

Proposition 10.1 The European option component of the American call option can be written as

$$
V_{E}\left(\tau, S, v_{1}, v_{2}\right)=e^{-q \tau} S P_{1}\left(\tau, S, v_{1}, v_{2} ; K\right)-e^{-r \tau} K P_{2}\left(\tau, S, v_{1}, v_{2} ; K\right),
$$

where

$$
P_{j}\left(\tau, S, v_{1}, v_{2} ; K\right)=\frac{1}{2}+\frac{1}{\pi} \int_{0}^{\infty} \operatorname{Re}\left(\frac{g_{j}\left(\tau, S, v_{1}, v_{2} ; \eta\right) e^{-i \eta \ln K}}{i \eta}\right) d \eta
$$


for $j=1,2$ with

$$
\begin{gathered}
g_{j}\left(\tau, S, v_{1}, v_{2} ; \eta\right)=\exp \left\{i \eta \ln S+B_{j}(\tau, \eta)+D_{1, j}(\tau, \eta) v_{1}+D_{2, j}(\tau, \eta) v_{2}\right\} \\
B_{j}(\tau, \eta)=i \eta(r-q) \tau+\frac{\Phi_{1}}{\sigma_{1}^{2}}\left\{\left(\Theta_{1, j}+\Omega_{1, j}\right) \tau-2 \ln \left(\frac{1-Q_{1, j} e^{\Omega_{1, j} \tau}}{1-Q_{1, j}}\right)\right\} \\
\quad+\frac{\Phi_{2}}{\sigma_{2}^{2}}\left\{\left(\Theta_{2, j}+\Omega_{2, j}\right) \tau-2 \ln \left(\frac{1-Q_{2, j} e^{\Omega_{2, j} \tau}}{1-Q_{2, j}}\right)\right\} \\
D_{1, j}(\tau, \eta)=\frac{\left(\Theta_{1, j}+\Omega_{1, j}\right)}{\sigma_{1}^{2}}\left[\frac{1-e^{\Omega_{1, j} \tau}}{1-Q_{1, j} e^{\Omega_{1, j} \tau}}\right] \\
D_{2, j}(\tau, \eta)=\frac{\left(\Theta_{2, j}+\Omega_{2, j}\right)}{\sigma_{2}^{2}}\left[\frac{1-e^{\Omega_{2, j} \tau}}{1-Q_{2, j} e^{\Omega_{2, j} \tau}}\right]
\end{gathered}
$$

Here, $Q_{m, j}=\left(\Theta_{m, j}+\Omega_{m, j}\right) /\left(\Theta_{m, j}-\Omega_{m, j}\right)$ for $m=1,2$ and $j=1,2$ where $\Theta_{1,1}=\Theta_{1}(i-\eta)$, $\Theta_{1,2}=\Theta_{1}(-\eta), \Theta_{2,1}=\Theta_{2}(i-\eta), \Theta_{2,2}=\Theta_{2}(-\eta), \Omega_{1,1}=\Omega_{1}(i-\eta), \Omega_{1,2}=\Omega_{1}(-\eta)$, $\Omega_{2,1}=\Omega_{2}(i-\eta)$ and $\Omega_{2,2}=\Omega_{2}(-\eta)$.

Proof: Refer to Appendix 8.

Remark 10.1 We recall that the definitions of $\Theta_{1}, \Theta_{2}, \Omega_{1}$ and $\Omega_{2}$ have been provided in equation (5.2). Also $\Phi_{1}$ and $\Phi_{2}$ have been defined in equation (3.6).

When numerically implementing this pricing function in Proposition 10.2, it is desirable to adopt the ideas proposed in Kahl and Jäckel (2005) and Albrecher, Mayer, Schoutens and Tistaert (2007). Such techniques prevent the possibilities of branch cuts ${ }^{6}$ and ensure that the density function is continuous in the complex plane. Discontinuities are frequently more pronounced when pricing long maturity options. An example to this effect can be found on Table 1 of Albrecher et al. (2007).

Proposition 10.2 The early exercise premium component of the American call option can be represented as

$$
\begin{gathered}
V_{P}\left(\tau, S, v_{1}, v_{2}\right)=\int_{0}^{\tau} \int_{0}^{\infty} \int_{0}^{\infty}\left[q e^{-q(\tau-\xi)} S P_{1}^{A}\left(\tau-\xi, S, v_{1}, v_{2} ; w_{1}, w_{2}, b\left(\xi, w_{1}, w_{2}\right)\right)\right. \\
\left.-r e^{-r(\tau-\xi)} K P_{2}^{A}\left(\tau-\xi, S, v_{1}, v_{2} ; w_{1}, w_{2}, b\left(\xi, w_{1}, w_{2}\right)\right)\right] d w_{1} d w_{2} d \xi
\end{gathered}
$$

where

$$
\begin{aligned}
P_{j}^{A}\left(\tau-\xi, S, v_{1}, v_{2} ; w_{1}, w_{2}, b\left(\xi, v_{1}, v_{2}\right)\right)=\frac{1}{2} \\
+\frac{1}{\pi} \int_{0}^{\infty} \operatorname{Re}\left(\frac{g_{j}^{A}\left(\tau-\xi, S, v_{1}, v_{2} ; \eta, w_{1}, w_{2}\right) e^{-i \eta \ln b\left(\xi, w_{1}, w_{2}\right)}}{i \eta}\right) d \eta
\end{aligned}
$$

\footnotetext{
${ }^{6} \mathrm{~A}$ branch cut is a curve in the complex plane across which a function is discontinuous.
} 


$$
\begin{aligned}
& \text { for } j=1,2 \text { with } \\
& \begin{aligned}
g_{j}^{A}\left(\tau-\xi, S, v_{1}, v_{2} ; \eta, w_{1}, w_{2}\right)=\exp \left\{\left(\frac{\Theta_{1, j}-\Omega_{1, j}}{\sigma_{1}^{2}}\right)\left(v_{1}-w_{1}+\Phi_{1}(\tau-\xi)\right)\right. \\
\left.\quad+\left(\frac{\Theta_{2, j}-\Omega_{2, j}}{\sigma_{2}^{2}}\right)\left(v_{2}-w_{2}+\Phi_{2}(\tau-\xi)\right)\right\} \\
\times \exp \left\{-\left(\frac{2 \Omega_{1, j}}{\sigma_{1}^{2}\left(e^{\Omega_{1, j}(\tau-\xi)}-1\right)}\right)\left(w_{1} e^{\Omega_{1, j}(\tau-\xi)}+v_{1}\right)-\left(\frac{2 \Omega_{2, j}}{\sigma_{2}^{2}\left(e^{\Omega_{2, j}(\tau-\xi)}-1\right)}\right)\left(w_{2} e^{\Omega_{2, j}(\tau-\xi)}+v_{2}\right)\right\} \\
\times e^{i \eta \ln S+i \eta(r-q)(\tau-\xi)} \frac{2 \Omega_{1, j} e^{\Omega_{1, j}(\tau-\xi)}}{\sigma_{1}^{2}\left(e^{\Omega_{1}(\tau-\xi)}-1\right)} \frac{2 \Omega_{2, j} e^{\Omega_{2, j}(\tau-\xi)}}{\sigma_{2}^{2}\left(e^{\Omega_{2, j}(\tau-\xi)}-1\right)}\left(\frac{w_{1} e^{\Omega_{1, j}(\tau-\xi)}}{v_{1}}\right)^{\frac{\Phi_{1}}{\sigma_{1}^{2}}-\frac{1}{2}}\left(\frac{w_{2} e^{\Omega_{2, j}(\tau-\xi)}}{v_{2}}\right)^{\frac{\Phi_{2}}{\sigma_{2}^{2}}-\frac{1}{2}} \\
\times I_{\frac{2 \Phi_{1}}{\sigma_{1}^{2}}-1}\left(\frac{4 \Omega_{1, j}}{\sigma_{1}^{2}\left(e^{\Omega_{1, j}(\tau-\xi)}-1\right)}\left(v_{1} w_{1} e^{\Omega_{1, j}(\tau-\xi)}\right)^{\frac{1}{2}}\right) I_{\frac{\Phi_{\Phi_{2}}}{\sigma_{2}^{2}}-1}\left(\frac{4 \Omega_{2, j}}{\sigma_{2}^{2}\left(e^{\Omega_{2, j}(\tau-\xi)}-1\right)}\left(v_{2} w_{2} e^{\Omega_{2, j}(\tau-\xi)}\right)^{\frac{1}{2}}\right) .
\end{aligned}
\end{aligned}
$$

The expressions for $\Theta_{m, j}$ and $\Omega_{m, j}$ are given in Proposition 10.1 above.

Proof: Refer to Appendix 9.

Equation (10.1) is in terms of the early exercise boundary, $b\left(\tau, v_{1}, v_{2}\right)$ which is still unknown. This function needs to be determined for us to have the corresponding option price at each point in time. Also, the three integrals of the early exercise premium component cannot be integrated out as we do not know the functional form of this early exercise boundary. The only knowledge we have is that it is a function of time and the two instantaneous variances. The early exercise boundary also satisfies the value matching condition

$$
b\left(\tau, v_{1}, v_{2}\right)-K=V\left(\tau, b\left(\tau, v_{1}, v_{2}\right), v_{1}, v_{2}\right),
$$

which, given the integral expression for $V\left(\tau, b\left(\tau, v_{1}, v_{2}\right), v_{1}, v_{2}\right)$ is a non-linear Volterra integral equation. This can be solved directly for the free-boundary but we seek some approximation techniques in order to reduce the computational burden associated with solving the integral equations directly. We present one approximation technique for the early exercise boundary in the next section.

\section{Approximating the Early Exercise Surface}

The idea of approximating early exercise boundaries has gained popularity in pricing standard ${ }^{7}$ American options; Ju (1998) uses multi-piece exponential functions to approximate the early exercise boundary of the American put option. Chiarella, El-Hassan and Kucera (1999) use Fourier-Hermite series expansions to represent the underlying asset price evolution and then present a systematic approach for evaluating the corresponding options written on a particular underlying asset. Ait-Sahlia and Lai (2001) approximate Kim's (1990) early exercise boundary with a piecewise linear function. They first discretise the time domain into

\footnotetext{
${ }^{7}$ Here, the term "standard" means an option pricing model that satisfies all Black and Scholes (1973) assumptions.
} 
equally spaced sub-intervals. Linear interpolation is then incorporated to fit the early exercise boundary between two successive subintervals thereby generating the entire free-boundary. Mallier (2002) consider series solutions for the location of the early exercise boundary close to expiry.

Approximation techniques have also been generalised to American options under stochastic volatility. Broadie et al. (2000) have shown empirically in the single stochastic volatility case that $\ln b(\tau, v)$ can well be approximated by a function that is linear in $v$. Based on these empirical findings, Tzavalis and Wang (2003) have expanded the logarithm of the early exercise boundary using Taylor series around the long-run volatility. This expansion yields two unknown functions of time which they later determine using Chebyshev polynomial expansion techniques. Instead of applying a Chebyshev approximation, Adolfsson et al. (2009) use numerical integration techniques and root finding methods to find these unknown functions of time. This method proves to be adequate enough in terms of accuracy and computational speed as compared to other valuation methods that they consider. It is this approach that we employ in this paper.

We first use a Taylor series expansion to expand the logarithm of the early exercise boundary, $\ln b\left(\tau, v_{1}, v_{2}\right)$ around the corresponding long-run variances such that

$$
\ln b\left(\tau, v_{1}, v_{2}\right) \approx b_{0}(\tau)+b_{1}(\tau) v_{1}+b_{2}(\tau) v_{2}
$$

where $b_{0}(\tau), b_{1}(\tau)$ and $b_{2}(\tau)$ are functions of time to be determined. This approach allow us to simplify the two integrals with respect to $w_{1}$ and $w_{2}$ in equation (10.1) before applying the numerical algorithm. Incorporating the expansion (11.1) into equation (10.5) we obtain the results in the proposition below.

Proposition 11.1 By approximating the early exercise boundary with the expression

$$
\ln b\left(\tau, v_{1}, v_{2}\right) \approx b_{0}(\tau)+b_{1}(\tau) v_{1}+b_{2}(\tau) v_{2}
$$

the value of the American call option can be re-expressed as

$$
V\left(\tau, S, v_{1}, v_{2}\right) \approx V_{E}\left(\tau, S, v_{1}, v_{2}\right)+V_{P}^{A}\left(\tau, S, v_{1}, v_{2}\right)
$$

where $V_{E}\left(\tau, S, v_{1}, v_{2}\right)$ is as presented in Proposition 10.1 and the approximation to the early exercise premium is given by

$$
\begin{aligned}
V_{P}^{A}\left(\tau, S, v_{1}, v_{2}\right)= & \int_{0}^{\tau}\left[q e^{-q(\tau-\xi)} S \hat{P}_{1}^{A}\left(\tau-\xi, S, v_{1}, v_{2} ; b_{0}(\xi), b_{1}(\xi), b_{2}(\xi)\right)\right. \\
& \left.-r e^{-r(\tau-\xi)} K \hat{P}_{2}^{A}\left(\tau-\xi, S, v_{1}, v_{2} ; b_{0}(\xi), b_{1}(\xi), b_{2}(\xi)\right)\right] d \xi,
\end{aligned}
$$


where

$$
\begin{aligned}
\hat{P}_{j}^{A}\left(\tau-\xi, S, v_{1}, v_{2} ; b_{0}(\xi), b_{1}(\xi), b_{2}(\xi)\right)=\frac{1}{2} \\
\quad+\frac{1}{\pi} \int_{0}^{\infty} \operatorname{Re}\left(\frac{\hat{g}_{j}^{A}\left(\tau-\xi, S, v_{1}, v_{2} ; \eta, b_{1}(\xi), b_{2}(\xi)\right) e^{-i \eta b_{0}(\xi)}}{i \eta}\right) d \eta
\end{aligned}
$$

for $j=1,2$ with

$$
\begin{aligned}
& \hat{g}_{j}^{A}\left(\tau, S, v_{1}, v_{2} ; \eta, b_{1}, b_{2}\right)=\exp \left\{i \eta \ln S+B_{j}^{A}\left(\tau, \eta, b_{1}, b_{2}\right)+D_{1, j}^{A}\left(\tau, \eta, b_{1}\right) v_{1}+D_{2, j}^{A}\left(\tau, \eta, b_{2}\right) v_{2}\right\} \\
& \begin{aligned}
B_{j}^{A}\left(\tau, \eta, b_{1}, b_{2}\right)=i \eta(r-q) \tau+\frac{\Phi_{1}}{\sigma_{1}^{2}}\left\{\left(\Theta_{1, j}+\Omega_{1, j}\right) \tau-2 \ln \left(\frac{1-Q_{1, j}^{A} e^{\Omega_{1, j} \tau}}{1-Q_{1, j}^{A}}\right)\right\} \\
+\frac{\Phi_{2}}{\sigma_{2}^{2}}\left\{\left(\Theta_{2, j}+\Omega_{2, j}\right) \tau-2 \ln \left(\frac{1-Q_{2, j}^{A} e^{\Omega_{2, j} \tau}}{1-Q_{2, j}^{A}}\right)\right\}
\end{aligned} \\
& \begin{aligned}
D_{1, j}^{A}\left(\tau, \eta, b_{1}\right)=-i \eta b_{1}+\frac{\left(\Theta_{1, j}+\Omega_{1, j}\right)}{\sigma_{1}^{2}}\left[\frac{1-e^{\Omega_{1, j} \tau}}{1-Q_{1, j}^{A} e^{\Omega_{1, j} \tau}}\right] \\
D_{2, j}^{A}\left(\tau, \eta, b_{2}\right)=-i \eta b_{2}+\frac{\left(\Theta_{2, j}+\Omega_{2, j}\right)}{\sigma_{2}^{2}}\left[\frac{1-e^{\Omega_{2, j} \tau}}{1-Q_{2, j}^{A} e^{\Omega_{2, j} \tau}}\right] .
\end{aligned}
\end{aligned}
$$

Here

$$
Q_{m, j}^{A}=\frac{\Theta_{m, j}+i \eta \sigma_{m}^{2} b_{m}+\Omega_{m, j}}{\Theta_{m, j}+i \eta \sigma_{m}^{2} b_{m}-\Omega_{m, j}}, \quad \Theta_{m, j}=\Theta_{j}(i-\eta), \quad \text { and } \quad \Omega_{m, j}=\Omega_{j}(i-\eta),
$$

for $m=1,2$ and $j=1,2$.

Proof: Refer to Appendix 10.

By using the approximation (11.1), we have managed to reduce the number of integral dimensions from four to two as we have simplified the two integrals with respect to $w_{1}$ and $w_{2}$ in equation (10.5). The simplified version of the early exercise premium component enhances computational speed of our numerical scheme for finding both the early exercise boundary and the corresponding option price as the resulting equation is now independent of the modified Bessel functions which tends to consume much computational time. Given the approximation in equation (11.1), the value-matching condition can also be expressed as

$$
e^{b_{0}(\tau)+b_{1}(\tau) v_{1}+b_{2}(\tau) v_{2}}-K=V\left(\tau, e^{b_{0}(\tau)+b_{1}(\tau) v_{1}+b_{2}(\tau) v_{2}}, v_{1}, v_{2}\right)
$$

Equation (11.6) is implicit in $b_{0}(\tau), b_{1}(\tau)$ and $b_{2}(\tau)$, hence root finding techniques need to be employed for us to obtain explicit forms of these functions. In determining these functions, 
we formulate three equations such that

$$
\begin{aligned}
& b_{0}(\tau)=\ln \left[V\left(\tau, e^{b_{0}(\tau)+b_{1}(\tau) v_{1}+b_{2}(\tau) v_{2}}, v_{1}, v_{2}\right)+K\right]-b_{1}(\tau) v_{1}-b_{2}(\tau) v_{2}, \\
& b_{1}(\tau)=\frac{1}{v_{1}}\left(\ln \left[V\left(\tau, e^{b_{0}(\tau)+b_{1}(\tau) v_{1}+b_{2}(\tau) v_{2}}, v_{1}, v_{2}\right)+K\right]-b_{0}(\tau)-b_{2}(\tau) v_{2}\right), \\
& b_{2}(\tau)=\frac{1}{v_{2}}\left(\ln \left[V\left(\tau, e^{b_{0}(\tau)+b_{1}(\tau) v_{1}+b_{2}(\tau) v_{2}}, v_{1}, v_{2}\right)+K\right]-b_{0}(\tau)-b_{1}(\tau) v_{1}\right) .
\end{aligned}
$$

These equations need to be solved iteratively at each instant, details of which are outlined in the next section.

\section{Numerical Implementation}

Having derived the integral expression for the American call option price in equation (11.3) and the corresponding system of equations (11.7) for approximating the early exercise boundary, we now present the numerical algorithm for the implementation of these equations. A variety of techniques have been proposed in the literature for numerically solving equations like (11.7). Huang, Subrahmanyam and Yu (1996) use a numerical integration scheme to solve the Kim (1990) American put integral equation. Kallast and Kivinukk (2003) also use quadrature methods to approximate the price, delta, gamma and vega of both American call and put options. Adolfsson et al. (2009) use similar techniques to implement the integral expression for the American call option price when the dynamics of the underlying asset evolve under the influence of a stochastic variance process of the Heston (1993) type. In implementing our pricing algorithm, we shall use quadrature techniques as applied in Kallast and Kivinukk (2003).

The European option component of equation (11.3) involves only one integral with respect to the Fourier transform variable, this integration is easily handled by standard methods. However, the early exercise premium component has two integrals, one with respect to the Fourier transform variable and the other with respect to running time-to-maturity, $\xi$. The integral with respect to the Fourier transform variable is handled in a similar way as in the European component case. However, the integral with respect to $\xi$ requires the entire history of the three functions, $b_{0}(\tau), b_{1}(\tau)$, and $b_{2}(\tau)$ up to and including the current time. We therefore need to devise an algorithm to determine these three functions iteratively at each point in time.

In implementing equation (11.3) and the system (11.7), we treat the American option as a Bermudan option. The time interval is partitioned into $M$-equally spaced subintervals of length $h=T / M$. The algorithm is initiated at maturity and we then progress backwards in time. We denote the starting point as, $\tau_{0}=0$ which corresponds to maturity time. At maturity, it has been shown in Kim (1990) that the early exercise boundary of the American 
call option takes the form

$$
b\left(0, v_{1}, v_{2}\right)=\max \left(\frac{r}{q} K, K\right) .
$$

By comparing coefficients, we can readily deduce that

$$
b_{0}(0)=\max \left(\ln K, \ln \left[\frac{r}{q} K\right]\right), \quad b_{1}(0)=0, \quad \text { and } \quad b_{2}(0)=0
$$

All other time steps are denoted as $\tau_{m}=m h$, for $m=1,2, \cdots, M$. The discretised version of the American call option price is thus

$$
V\left(m h, S, v_{1}, v_{2}\right) \approx V_{E}\left(m h, S, v_{1}, v_{2}\right)+V_{P}^{A}\left(m h, S, v_{1}, v_{2}\right) .
$$

At each subsequent time step we need to determine the three unknown boundary terms, $b_{0}^{m}=b_{0}(m h), b_{1}^{m}=b_{1}(m h)$ and $b_{2}^{m}=b_{2}(m h)$ each of which depends on the entire early exercise boundary history. We use iterative techniques to find the values of these three unknown functions at each time step, that is, when iterating for $b_{0}^{m}, b_{1}^{m}$ and $b_{2}^{m}$, we use as initial guesses ${ }^{8} b_{0,0}^{m}=b_{0}^{m-1}, b_{1,0}^{m}=b_{1}^{m-1}$ and $b_{2,0}^{m}=b_{2}^{m-1}$ followed by solving the system of linked equations

$$
\begin{aligned}
& b_{0, k}^{m}=\ln \left[V\left(m h, e^{b_{0, k}^{m}+b_{1, k-1}^{m} v_{1}+b_{2, k-1}^{m} v_{2}}, v_{1}, v_{2}\right)+K\right]-b_{1, k-1}^{m} v_{1}-b_{2, k-1}^{m} v_{2}, \\
& b_{1, k}^{m}=\frac{1}{v_{1}}\left(\ln \left[V\left(m h, e^{b_{0, k}^{m}+b_{1, k}^{m} v_{1}+b_{2, k-1}^{m} v_{2}}, v_{1}, v_{2}\right)+K\right]-b_{0, k}^{m}-b_{2, k-1}^{m} v_{2}\right), \\
& b_{2, k}^{m}=\frac{1}{v_{2}}\left(\ln \left[V\left(m h, e^{b_{0, k}^{m}+b_{1, k}^{m} v_{1}+b_{2, k}^{m} v_{2}}, v_{1}, v_{2}\right)+K\right]-b_{0, k}^{m}-b_{1, k}^{m} v_{1}\right) .
\end{aligned}
$$

We find the value of $k$ such that $\left|b_{0, k}^{m}-b_{0, k-1}^{m}\right|<\epsilon_{0},\left|b_{1, k}^{m}-b_{1, k-1}^{m}\right|<\epsilon_{1}$ and $\left|b_{2, k}^{m}-b_{2, k-1}^{m}\right|<\epsilon_{2}$, where $\epsilon_{0}, \epsilon_{1}$ and $\epsilon_{2}$ are tolerance values. Once the tolerance values are attained, we then proceed to the next time step. This algorithm is applicable to any root finding method for determining the triplet, $b_{0}, b_{1}$ and $b_{2}$. Adolfsson et al. (2009) use Newton's method while we prefer to use the bisection method in this paper as it does not involve the computation of the first derivative of the pricing function.

In the next section we present numerical and graphical results for the early exercise boundary and the corresponding American call option prices obtained using the above approach. We also provide graphs for the joint probability density functions of the state variables. Such density functions are crucial as they give us a clue on how to handle the unbounded integral domains of the state variables and address the convergence property of density functions.

\footnotetext{
${ }^{8}$ The subscript $k$ in the three functions $b_{0, k}^{m}, b_{1, k}^{m}$ and $b_{2, k}^{m}$ represents the number of iterations required for convergence of the iterative process at time step $m$.
} 


\section{Numerical Results}

Having presented the numerical algorithm as outlined above, we now provide some numerical examples in this section. In what follows, we will dub our method the numerical integration scheme. We have also implemented the Method of Lines (MOL) algorithm for the PDE (2.21) for comparison purposes. Details on how to implement the MOL algorithm can be found in Chiarella, Kang, Meyer and Ziogas (2009) where they consider the valuation of American options under stochastic volatility and jump diffusion processes. In all the numerical experiments that follow unless otherwise stated, we will use the parameters provided in Table 1 where, $v_{1}^{\max }$ and $v_{2}^{\max }$ are the maximum levels of the two instantaneous variances under consideration. We have discretised the two variance domains into 30 equally spaced sub intervals and $M=200$ time steps. For the MOL algorithm a non-uniform grid is applied to the underlying asset domain and a total number of 1,438 grid points has been used. The large number of grid points in the underlying asset domain helps in stabilising the numerical scheme and enhancing the smoothness of the early exercise boundary. For the numerical scheme to be stable, we have used 40 grid points in the interval $0 \leq S \leq 1,198$ points in the interval $1<S \leq 100$ and 1200 points within the interval $100<S \leq 500$.

\begin{tabular}{cccccc}
\hline \hline Parameter & Value & $v_{1}-$ Parameter & Value & $v_{2}-$ Parameter & Value \\
\hline$K$ & 100 & $\theta_{1}$ & $6 \%$ & $\theta_{2}$ & $8 \%$ \\
$r$ & $3 \%$ & $\kappa_{1}$ & 3 & $\kappa_{2}$ & 4 \\
$q$ & $5 \%$ & $\sigma_{1}$ & $10 \%$ & $\sigma_{2}$ & $11 \%$ \\
$T$ & 0.5 & $\rho_{13}$ & \pm 0.5 & $\rho_{24}$ & \pm 0.5 \\
$M$ & 200 & $\lambda_{1}$ & 0 & $\lambda_{2}$ & 0 \\
& & $v_{1}^{\max }$ & $20 \%$ & $v_{2}^{\max }$ & $20 \%$ \\
\hline
\end{tabular}

Table 1: Parameters used for the American call option. The $v_{1}$ column contains parameters for the first variance process whilst the $v_{2}$ column contains parameters for the second variance process.

We start by presenting the joint probability density function of $S$ and $v_{1}$ when $v_{2}$ is fixed and that of $S$ and $v_{2}$ when $v_{1}$ is fixed in Figures 1 and 2 respectively. These surfaces are generated by implementing equation (9.3). The nature of these probability density functions guide us on how to truncate the infinite domains of the state variables when performing numerical integration experiments. From these figures we note that density functions are zero everywhere except near the origins of the state variables. For instance, instead of integrating the underlying asset domain from zero to infinity in our case we have simply integrated from zero to 50 since beyond this point the density function is extremely close to zero. Such diagrams also provide a natural way of analysing the distribution of asset returns under stochastic volatility.

Having established the integration domains for the state variables, we present in Figure 3 the early exercise surface for the American call option when $v_{2}$ if fixed. This surface shows how an increase in $v_{1}$ affects the free-boundary of the American call option. We note from this figure that the early exercise surface is an increasing function of $v_{1}$ and is of the form typical for that of an American call option written on a single underlying asset whose dynamics 
Joint PDF of $S$ and $v_{1}$ when $v_{2}=11 \%$

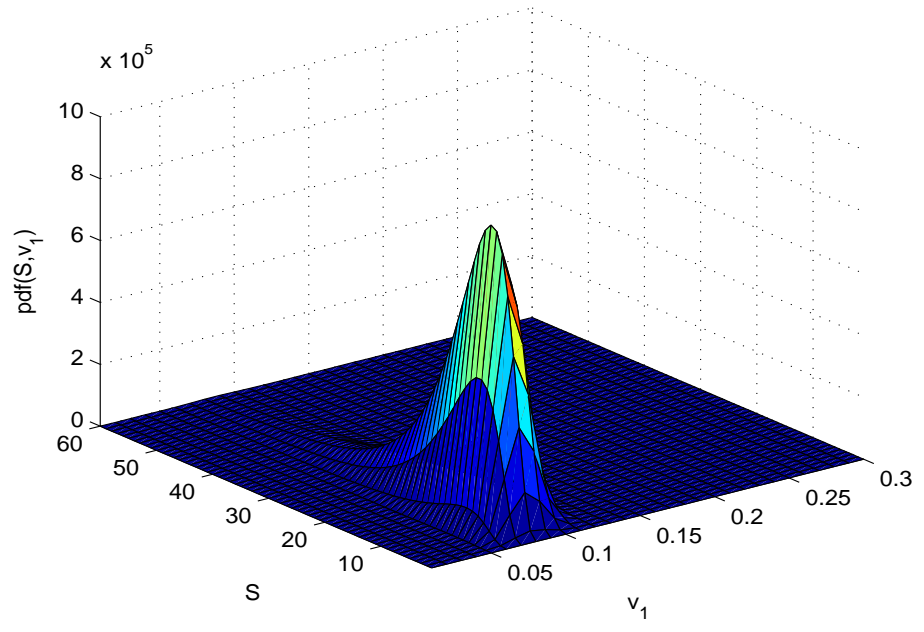

Figure 1: The probability density function of $S$ and $v_{1}$ when $v_{2}$ is fixed. We have considered the case when $\rho_{13}=0.5$ and $\rho_{24}=0.5$ with all other parameters as provided in Table 1 .

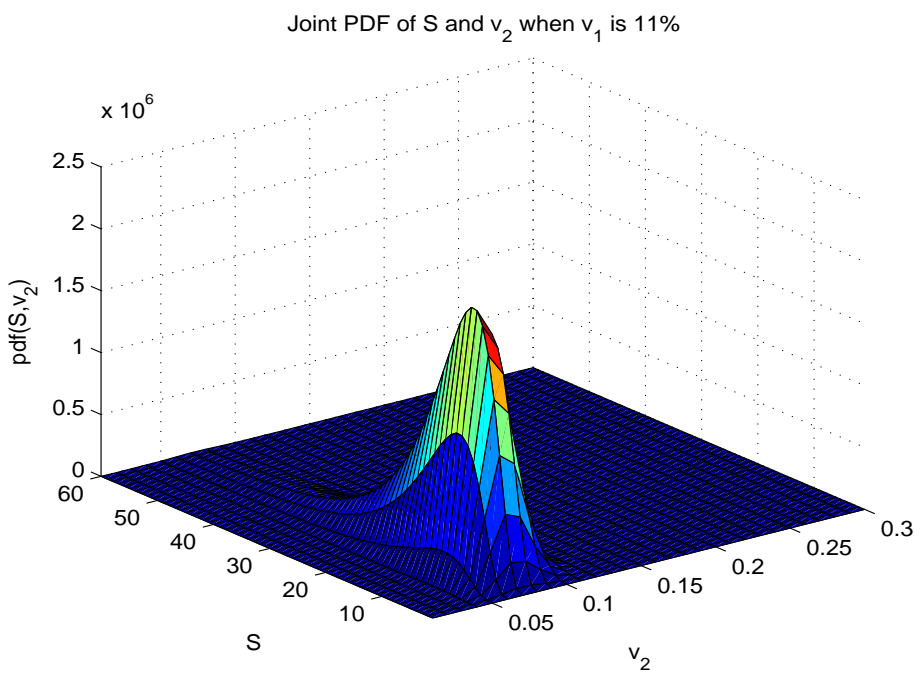

Figure 2: The probability density function of $S$ and $v_{2}$ when $v_{1}$ is fixed. We have considered the case when $\rho_{13}=0.5$ and $\rho_{24}=0.5$ with all other parameters as provided in Table 1 . 
evolve under the influence of a single stochastic variance process as presented in Chiarella et al. (2009). A similar surface can be obtained by fixing $v_{1}$ and allowing $v_{2}$ to vary.

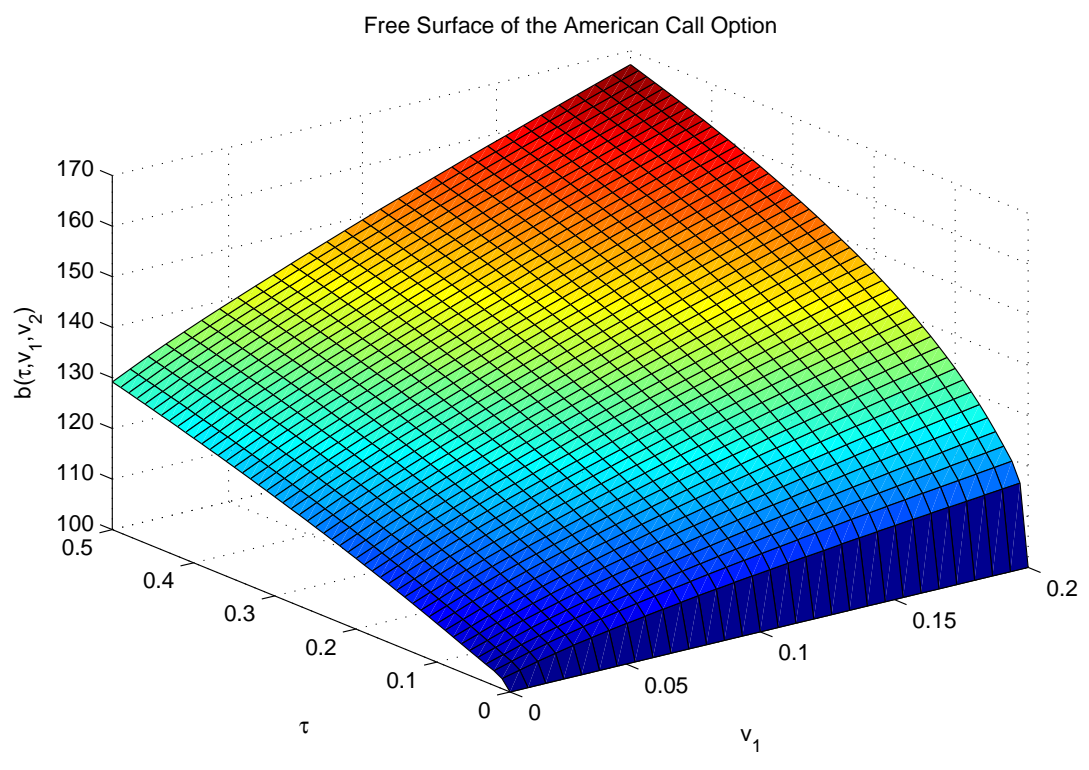

Figure 3: Early exercise surface of the American Call option when $v_{2}=0.67 \%, \rho_{13}=0.5$ and $\rho_{24}=0.5$. All other parameters are as presented in Table 1 .

We can also compare the early exercise boundaries for the American call option when both $v_{1}$ and $v_{2}$ are fixed. Figure 4 shows these comparisons for varying correlation coefficients. Note from this figure that for fixed $v_{1}$ and $v_{2}$, early exercise boundaries typical for standard American call options are generated. We have also included the free-boundary generated from the geometric Brownian motion (GBM) model to highlight the impact of stochastic volatility on the American call option free-boundary. Since the two instantaneous variance processes under consideration are mean reverting, we calculate the corresponding GBM constant standard deviation as

$$
\sigma_{G B M}=\sqrt{\theta_{1}+\theta_{2}}
$$

where $\theta_{1}$ and $\theta_{2}$ are the long run variances of $v_{1}$ and $v_{2}$ respectively. From Figure 4 we note that zero correlations almost correspond to the GBM case. The early exercise boundary generated when the correlations are negative lies above that of the GBM model whilst that for positive correlations lies below as revealed in Figure 4.

Figure 5 shows the effects of varying volatilities of $v_{1}$ and $v_{2}$ to the early exercise boundary. We note that increasing both $\sigma_{1}$ and $\sigma_{2}$ has the effect of lowering the exercise boundary. We have considered the case when both $\rho_{13}$ and $\rho_{24}$ are equal to 0.5 and the instantaneous variances equal to their long run means.

To justify the effectiveness of our approach in valuing American call options, we need to compare the results with other pricing methods. In Figure 6 we present the early exercise boundaries from the method of lines (MOL) algorithm and numerical integration respectively. 
From this diagram we note that the early exercise boundary generated by the numerical integration method is slightly lower than that from the MOL. This might be attributed to approximation and discretisation errors from the numerical integration method. Discretisation errors can be reduced by making the grids finer. Errors from early exercise boundary approximation can be reduced by devising better approximating functions empirically or by any other suitable approach. Similar comparisons can be made for different parameter combinations. We also present Figure 7 which compares the effects of different correlation coefficients on the same process. For example when $\rho_{13}=-0.5$ and $\rho_{24}=0.5$ we see that the corresponding early exercise boundary is slightly below the $\rho_{13}=0$ and $\rho_{24}=0$ boundary. This might be due to cancelation effect of the influential stochastic terms of the variance processes.

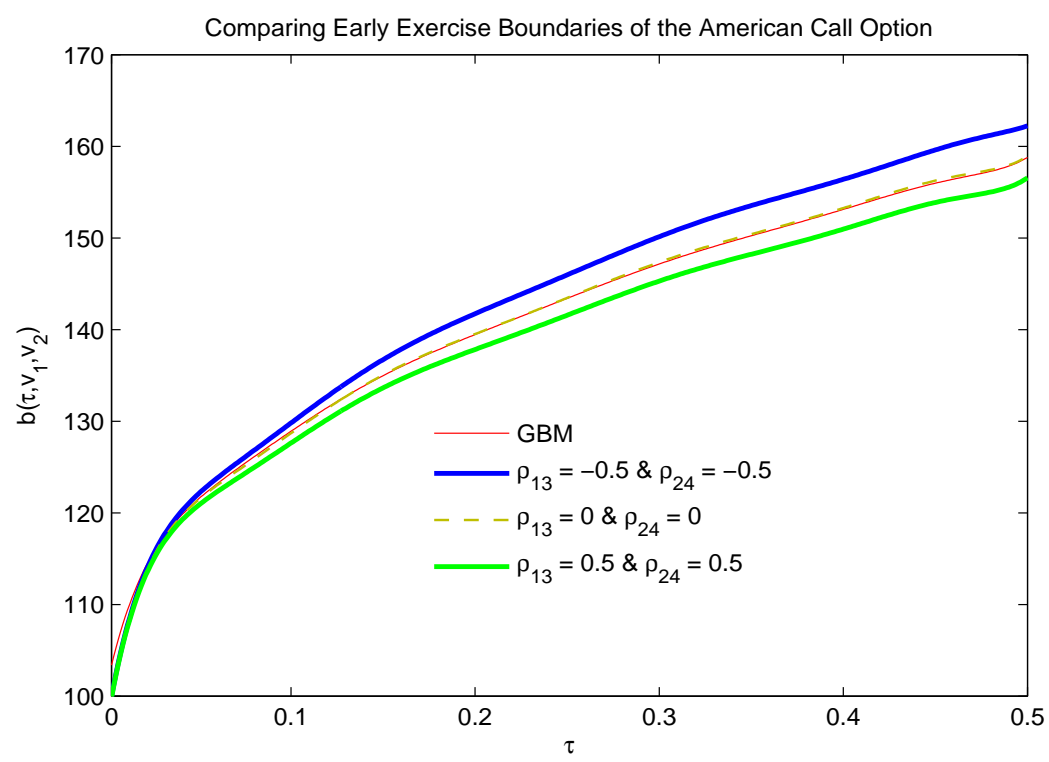

Figure 4: Exploring the effects of stochastic volatility on the early exercise boundary of the American call option for varying correlation coefficients when $\sigma_{G B M}=0.3742, v_{1}=6 \%$ and $v_{2}=8 \%$. All other parameters are provided in Table 1 .

We now turn to an analysis of option prices using the two approaches. Figure 8 shows the general American call option price surface at a fixed level of $v_{2}$. A similar surface can be generated by fixing $v_{1}$ and allowing $S$ and $v_{2}$ to vary. We can also assess the effects of stochastic volatility on the option prices for different correlation coefficients by making comparisons with GBM prices where we calculate the corresponding constant volatility using equation (13.1) which is the square-root of the average of the two long run variances. Figure 9 shows option price differences found by subtracting option prices from the numerical integration method from the corresponding GBM prices. As with the early exercise boundary comparisons, the zero correlation price differences are not significantly different from GBM prices. As documented in Heston (1993) and Chiarella et al. (2009), higher price differences are noted for far out-and in-the-money options. Positive correlations yield option prices which are lower than GBM prices for in-the-money options while generating prices which are higher for out-of-money options. The reverse effect holds for negative correlations. Higher price 


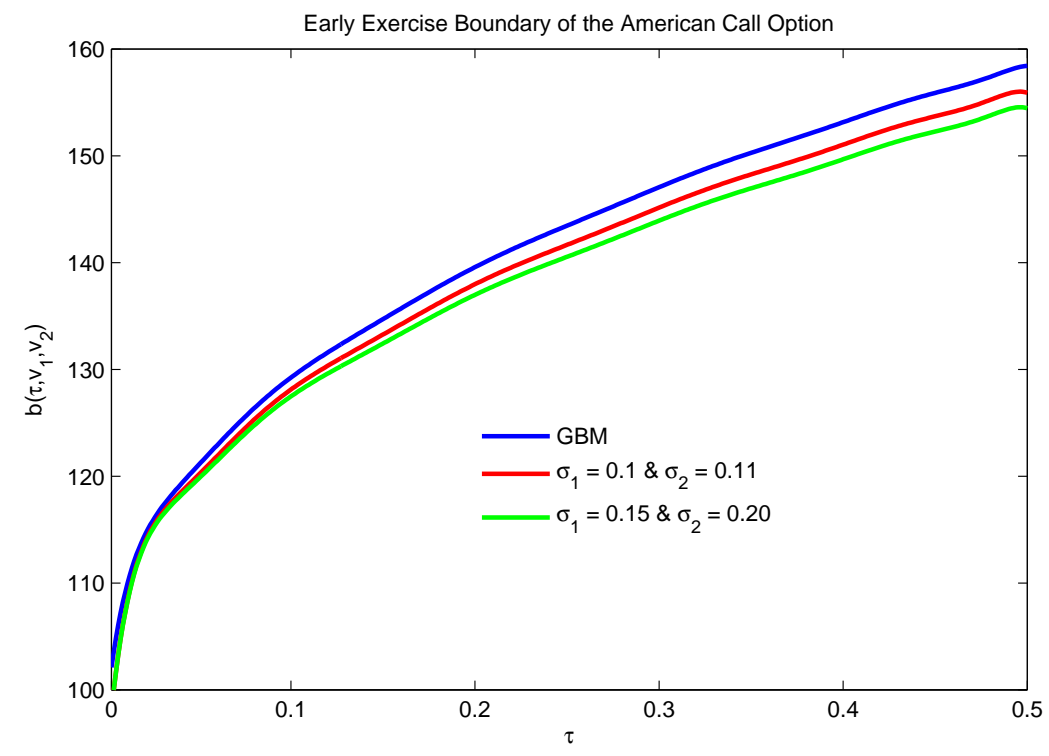

Figure 5: Exploring the effects of varying the volatilities of $v_{1}$ and $v_{2}$ on the early exercise boundary of the American call option. We have used the following parameters, $\sigma_{G B M}=$ $0.3742, v_{1}=6 \%, v_{2}=8 \%, \rho_{13}=0.5$ and $\rho_{24}=0.5$ with all other parameters as given in Table 1.

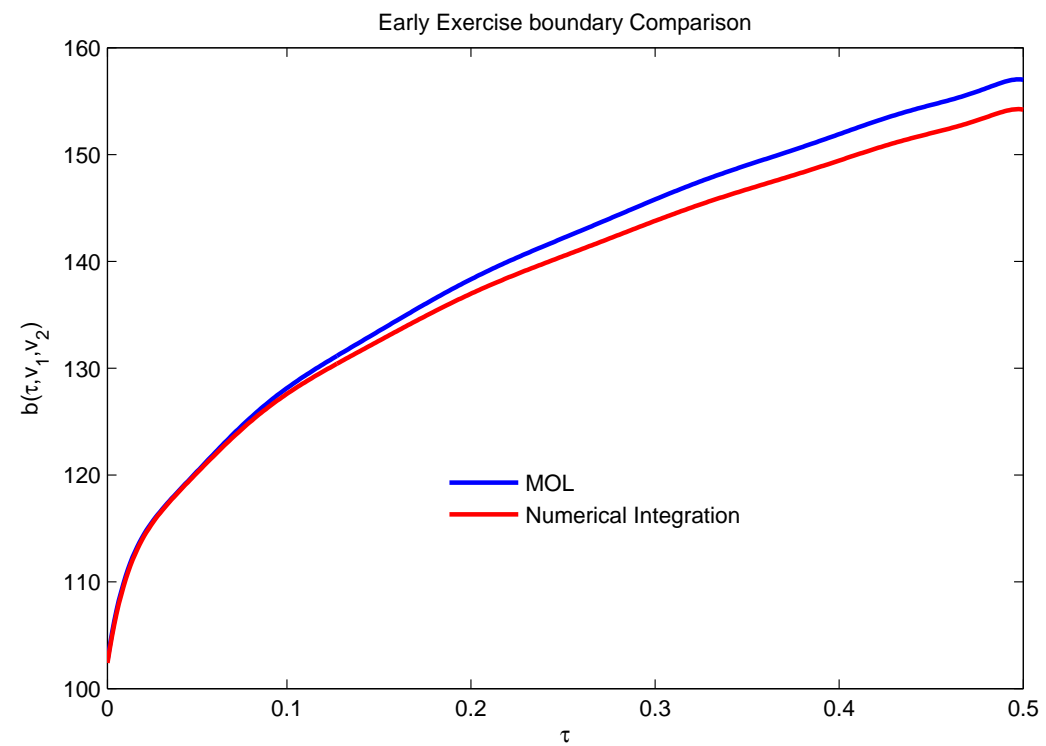

Figure 6: Comparing early exercise boundaries from the MOL and numerical integration approach when the two instantaneous variances are fixed. Here, $v_{1}=0.67 \%, v_{2}=13.33 \%$, $\rho_{13}=0.5$ and $\rho_{24}=0.5$ with all other parameters as given in Table 1 . 


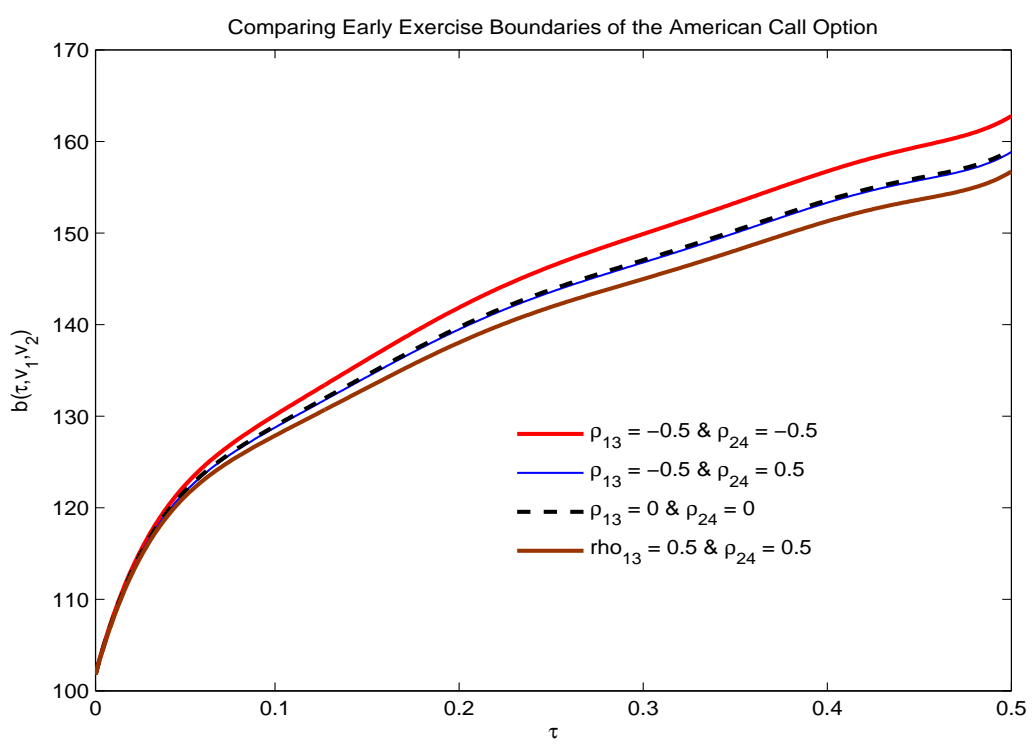

Figure 7: Exploring the effects of mixed correlation coefficients on the early exercise boundary of the American call option. We have used the following parameters, $v_{1}=6 \%, v_{2}=8 \%$ with all other parameters as given in Table 1.

differences of up to 0.1 are noted for both positive and negative correlations.

We also present option prices obtained from the MOL and numerical integration methods together with the associated GBM prices in Table 2 when $\rho_{13}=0.5$ and $\rho_{24}=0.5$. From this table we note that option prices obtained from the MOL and numerical integration methods are not significantly different from each other which shows that both methods are suitable for practical purposes in valuing American call options under stochastic volatility. We have included GBM prices to highlight the impact of stochastic volatility on option prices. When we presented numerical results for the early exercise boundaries we highlighted the effects of changes in the volatilities of $v_{1}$ and $v_{2}$, we also provide graphical results on how such changes affect option prices in Figure 10. In this figure, we have used the case when $\rho_{13}=0.5$ and $\rho_{24}=0.5$. We can readily see that higher price differences occur for higher $\sigma_{1}$ and $\sigma_{2}$ with all other parameters as provided in Table 1 . This implies that higher volatilities of $v_{1}$ and $v_{2}$ have the effect of increasing the variances which then results in higher price differences for inand out-of-the-money options relative to GBM prices. Similar conclusions have been derived in Heston (1993) when considering the European call option under stochastic volatility.

The most important feature of the MOL is that the option price, delta and the free-boundary are all generated simultaneously as part of the solution process at no added computational cost. Given such a tremendous convenience, we wrap up this section by presenting the delta surface of the American call option in Figure 11 for fixed $v_{2}$. A similar surface can be obtained by holding $v_{1}$ constant. We also explore the effects of stochastic volatility on the delta by making comparisons with the GBM delta in Figure 12. From this figure, we note that the option delta is very sensitive to the changes in the variance. 


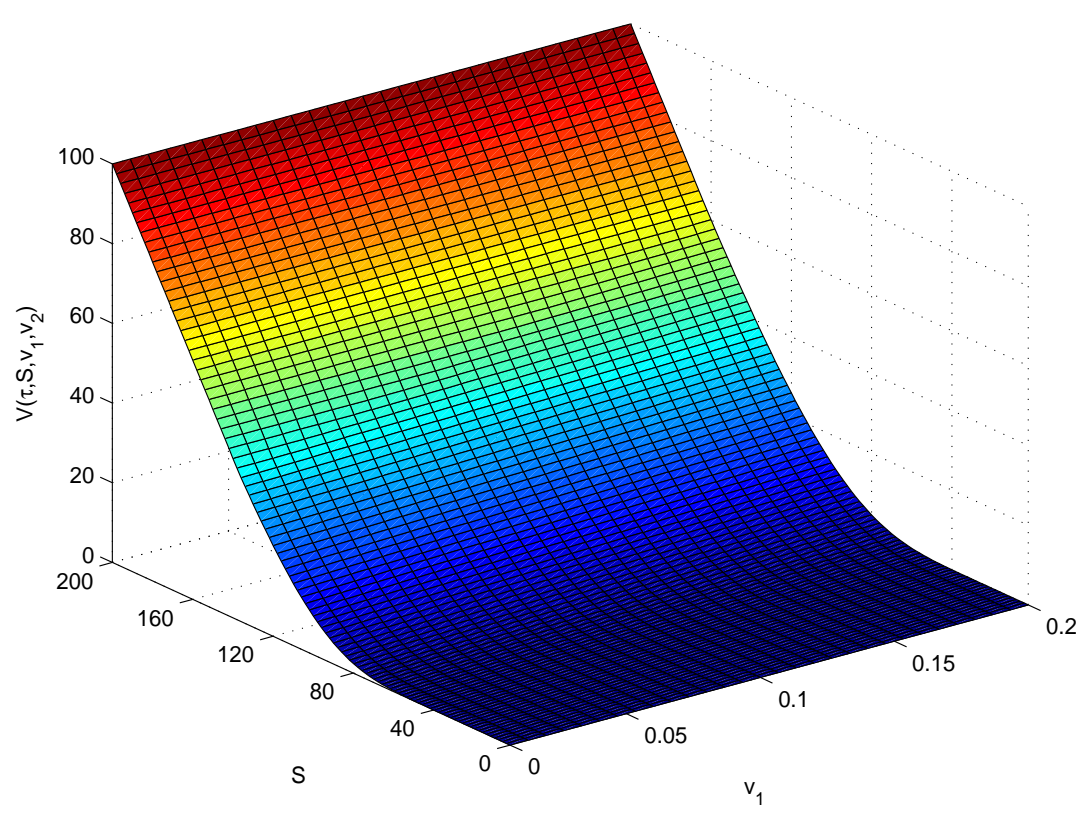

Figure 8: American call option price surface when $v_{2}=13.33 \%, \rho_{13}=0$ and $\rho_{24}=0$ with all other parameters provided in Table 1.

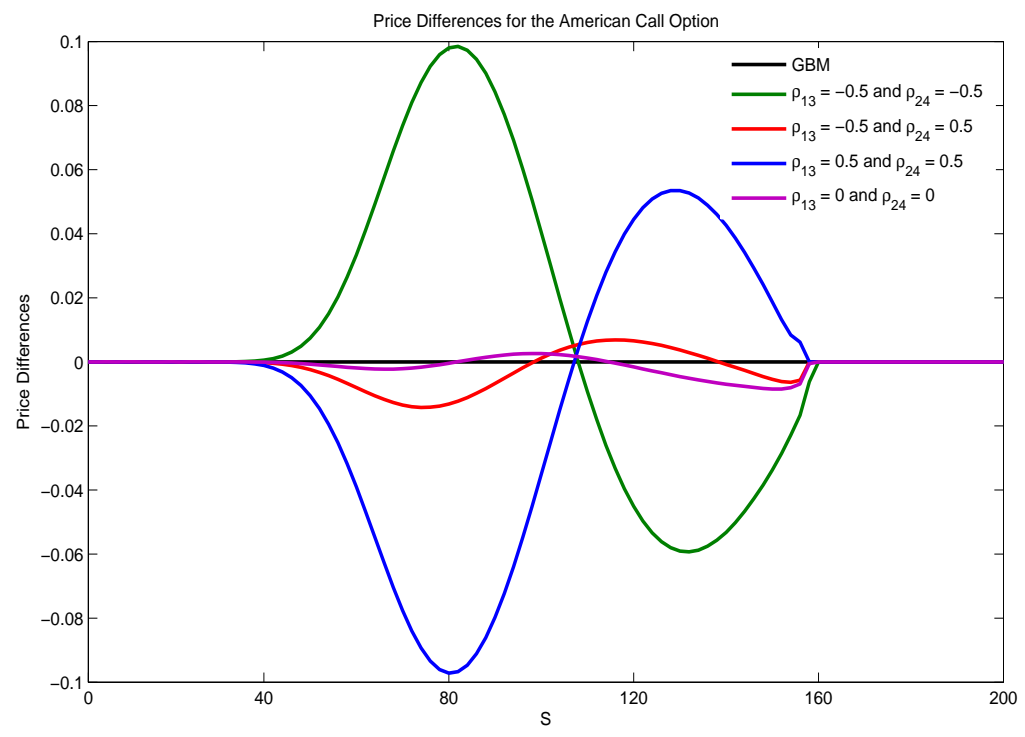

Figure 9: Option prices from the geometric Brownian motion minus option prices from the Stochastic volatility model for varying correlation coefficients. Here, $\sigma_{G B M}=0.3742, v_{1}=6 \%$ and $v_{2}=8 \%$ with all other parameters provided in Table 1 . 


\begin{tabular}{cccc}
\hline \hline$S$ & Numerical Integration & MOL & GBM \\
\hline 60 & 0.2036 & 0.2029 & 0.1850 \\
80 & 2.4088 & 2.400 & 2.4154 \\
100 & 9.8082 & 9.7918 & 9.9452 \\
120 & 23.1069 & 23.0920 & 23.3006 \\
140 & 40.4756 & 40.4686 & 40.5922 \\
160 & 60 & 60 & 60 \\
180 & 80 & 80 & 80 \\
200 & 100 & 100 & 100 \\
\hline
\end{tabular}

Table 2: American call option price comparisons when $v_{1}=0.67 \%, v_{2}=13.33 \%, \rho_{13}=0.5$, $\rho_{24}=0.5$. We have taken GBM volatility to be $\sigma_{G B M}=0.3741657$ and this is found by using equation (13.1).

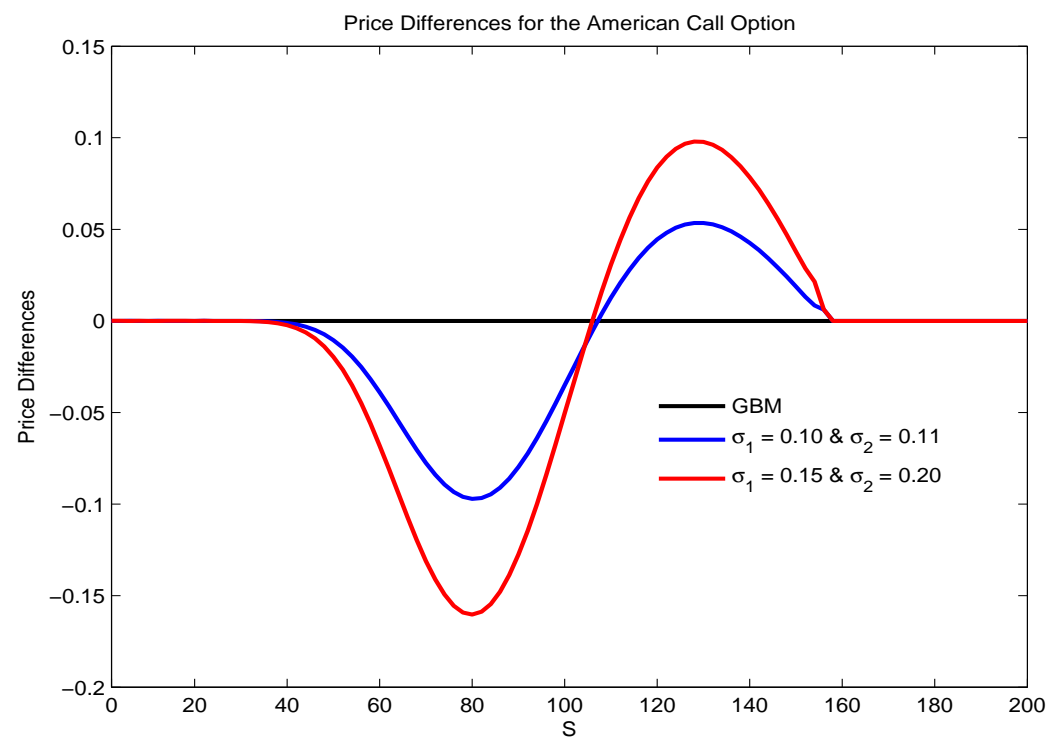

Figure 10: Option prices from the geometric Brownian motion minus option prices from the Stochastic volatility model for varying volatilities of volatility. Here, $\sigma_{G B M}=0.3742$, $v_{1}=6 \%, v_{2}=8 \%, \rho_{13}=0.5$ and $\rho_{24}=0.5$. All other parameters are provided in Table 1 . 


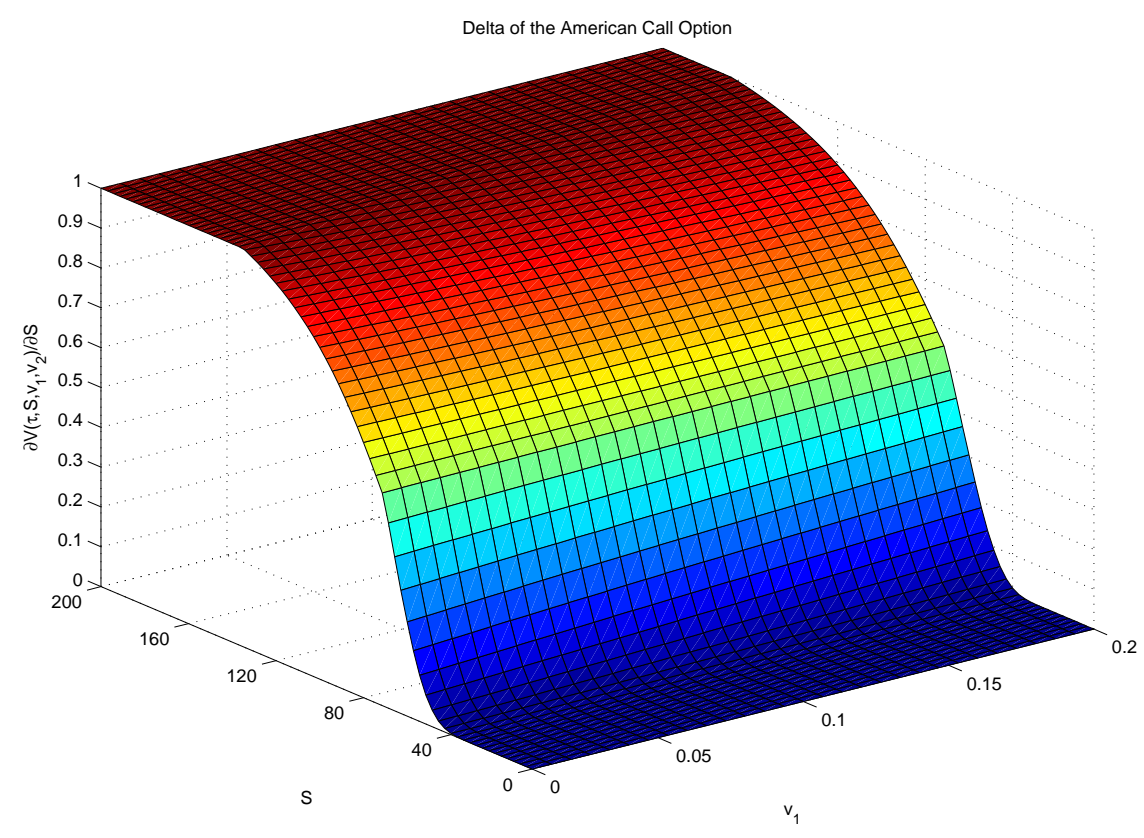

Figure 11: Delta surface of the American call option when $v_{2}=0.67 \%, \rho_{13}=0.5$ and $\rho_{24}=0.5$. All other parameters are as provided in Table 1 .

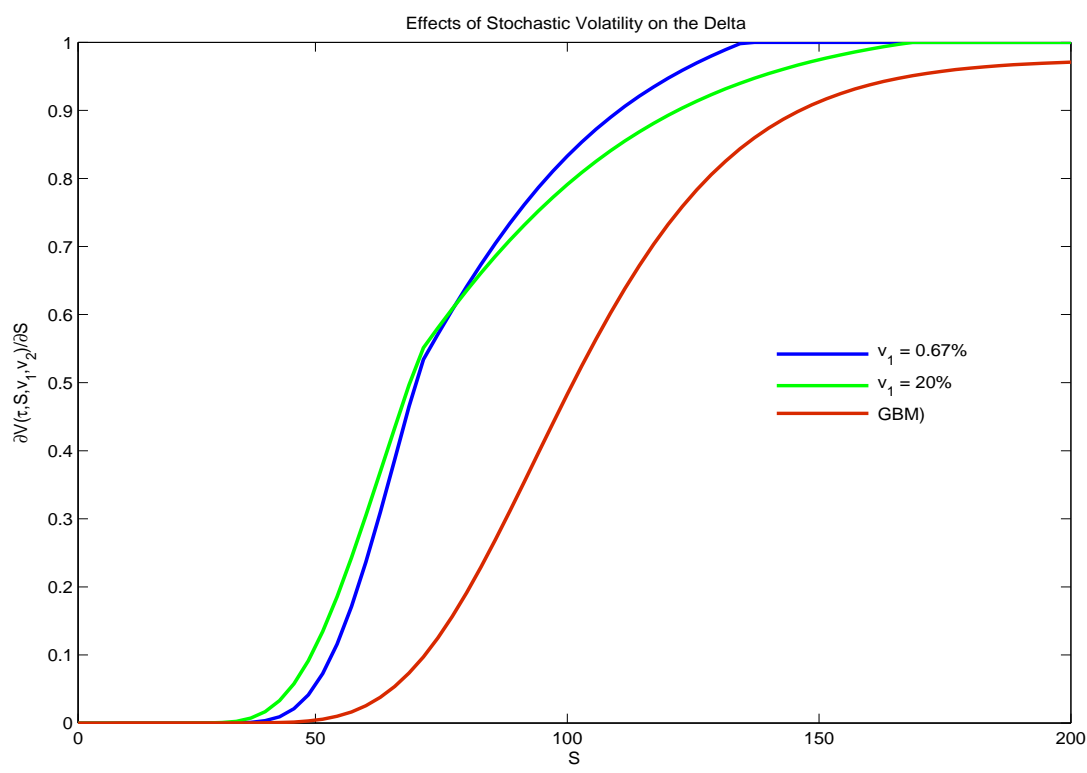

Figure 12: Exploring the effects of Stochastic volatility on the Delta of the American call option when $v_{2}=13.33 \%, \rho_{13}=0.5$ and $\rho_{24}=0.5$. All other parameters are as provided in Table 1. 


\section{Conclusion}

In this paper we have presented a numerical integration technique for pricing an American call option written on an underlying asset whose dynamics evolve under the influence of two stochastic variance processes of the Heston (1993) type. The approach involves the transformation of the pricing partial differential equation (PDE) to an inhomogeneous form by exploiting Jamshidian's (1992) techniques. An integral expression has been presented as the general solution of the inhomogeneous PDE with the aid of Duhamel's principle and this is a function of the transition density function.

The transition density function is a solution of the associated Kolmogorov backward PDE for the three stochastic processes under consideration. A systematic approach for solving the Kolmogorov PDE using a combination of Fourier and Laplace transforms has been presented. A means for numerically implementing the integral equation for the American call option has been provided. The early exercise boundary approximation has allowed a simplification of the double integrals with respect to the running variance variables. This reduces the computational burden when one proceeds to numerical implementation.

Numerical results exploring the impact of stochastic volatility on both option prices and the free-boundary have been provided and we have discovered that the correlations between the underlying asset and the two variance processes have a significant effect on in-and out-of-the money options. The numerical results presented yield similar findings of Heston (1993) and Chiarella et al. (2009) on the impact of stochastic volatility on option prices where they consider European and American option pricing under stochastic volatility respectively. We have also analysed the effects of varying the volatilities of instantaneous variances on both the early exercise boundary and the corresponding option prices. We note that an increase in the volatility of the instantaneous variances increases the corresponding variance levels resulting in higher price differences for in-and out-of-the-money options when compared with geometric Brownian motion prices.

We have assessed the accuracy of the numerical integration approach by making comparisons with numerical results from the method of lines (MOL) algorithm. Both approaches provide comparable results though there are slight differences on the early exercise boundary plots. Such differences are mainly due to early exercise boundary approximation and discretisation errors associated with the numerical integration method. As the MOL has an additional advantage of generating the option delta as part of the solution, we have exploited this feature and explored the impact of stochastic volatility on the American spread option delta generated by the Black and Scholes (1973) model.

The integral expression derived in this paper is applicable to any continuous payoff function, which is a powerful feature of Fourier and Laplace transform based methods. 


\section{Appendix 1. Proof of Proposition 3.1}

Consider the PDE

$$
\frac{\partial C}{\partial \tau}=\mathcal{D}_{x, v_{1}, v_{2}} C-r C+f\left(\tau, x, v_{1}, v_{2}\right)
$$

whose initial condition is the payoff at maturity, $C\left(0, x, v_{1}, v_{2}\right)=\left(e^{x}-K\right)^{+}$. The PDE (A1.1) is to be solved in the region $0 \leq \tau \leq T,-\infty \leq x<\infty$ and $0 \leq v_{1}, v_{2}<\infty$, and where we define the Dynkin operator $\mathcal{D}_{x, v_{1}, v_{2}}$ as

$$
\begin{aligned}
\mathcal{D}_{x, v_{1}, v_{2}} & =\left(r-q-\frac{1}{2} v_{1}-\frac{1}{2} v_{2}\right) \frac{\partial}{\partial x}+\Phi_{1} \frac{\partial}{\partial v_{1}}-\beta_{1} v_{1} \frac{\partial}{\partial v_{1}}+\Phi_{2} \frac{\partial}{\partial v_{2}}-\beta_{2} v_{2} \frac{\partial}{\partial v_{2}}+\frac{1}{2} v_{1} \frac{\partial^{2}}{\partial x^{2}} \\
& +\frac{1}{2} v_{2} \frac{\partial^{2}}{\partial x^{2}}+\rho_{13} \sigma_{1} v_{1} \frac{\partial^{2}}{\partial x \partial v_{1}}+\rho_{14} \sigma_{2} v_{2} \frac{\partial^{2}}{\partial x \partial v_{2}}+\frac{1}{2} \sigma_{1}^{2} v_{1} \frac{\partial^{2}}{\partial v_{1}^{2}}+\frac{1}{2} \sigma_{2}^{2} v_{2} \frac{\partial^{2}}{\partial v_{2}^{2}},
\end{aligned}
$$

with

$$
\Phi_{j}=\kappa_{j} \theta_{j} \quad \text { and } \quad \beta_{j}=\kappa_{j}+\lambda_{j}, \quad \text { for } \quad j=1,2
$$

By use of Duhamel principle, the solution of the PDE (A1.1) is given by

$$
\begin{gathered}
C\left(\tau, x, v_{1}, v_{2}\right)=e^{-r \tau} \int_{0}^{\infty} \int_{0}^{\infty} \int_{-\infty}^{\infty}\left(e^{u}-K\right)^{+} U\left(\tau, x, v_{1}, v_{2} ; u, w_{1}, w_{2}\right) d u d w_{1} d w_{2} \\
+\int_{0}^{\tau} e^{-r(\tau-\xi)} \int_{0}^{\infty} \int_{0}^{\infty} \int_{-\infty}^{\infty} f\left(\xi, u, w_{1}, w_{2}\right) U\left(\tau-\xi, x, v_{1}, v_{2} ; u, w_{1}, w_{2}\right) d u d w_{1} d w_{2} d \xi \\
\quad \equiv C_{E}\left(\tau, x, v_{1}, v_{2}\right)+C_{P}\left(\tau, x, v_{1}, v_{2}\right) .
\end{gathered}
$$

To verify that this is the correct solution, we will show that (A1.3) satisfies the PDE (A1.1). Substituting $C\left(\tau, x, v_{1}, v_{2}\right)=C_{E}\left(\tau, x, v_{1}, v_{2}\right)+C_{P}\left(\tau, x, v_{1}, v_{2}\right)$ into (A1.1) we proceed as follows:

$$
\begin{aligned}
\frac{\partial C}{\partial \tau}+ & r C-\mathcal{D}_{x, v_{1}, v_{2}} C-f\left(\tau, x, v_{1}, v_{2}\right) \\
= & e^{-r \tau} \int_{0}^{\infty} \int_{0}^{\infty} \int_{-\infty}^{\infty}\left(e^{u}-K\right)\left\{\frac{\partial U}{\partial \tau}-\mathcal{D}_{x, v_{1}, v_{2}} U\right\} d u d w_{1} d w_{2}-r C_{E}+r C_{E} \\
& +\int_{0}^{\infty} \int_{0}^{\infty} \int_{-\infty}^{\infty} f\left(\tau, u, w_{1}, w_{2}\right) U\left(0, x, v_{1}, v_{2} ; u, w_{1}, w_{2}\right) d u d w_{1} d w_{2} \\
& +\int_{0}^{\tau} e^{-r(\tau-\xi)} \int_{0}^{\infty} \int_{0}^{\infty} \int_{-\infty}^{\infty} f\left(\xi, u, w_{1}, w_{2}\right) \frac{\partial U}{\partial \tau} d u d w_{1} d w_{2} d \xi-r C_{P}+r C_{P} \\
& -\int_{0}^{\tau} e^{-r(\tau-\xi)} \int_{0}^{\infty} \int_{0}^{\infty} \int_{-\infty}^{\infty} f\left(\xi, u, w_{1}, w_{2}\right) \mathcal{D}_{x, v_{1}, v_{2}} U d u d w_{1} d w_{2} d \xi-f\left(\tau, x, v_{1}, v_{2}\right) \\
= & \int_{0}^{\infty} \int_{0}^{\infty} \int_{-\infty}^{\infty} f\left(\tau, x, v_{1}, v_{2}\right) \delta\left(e^{x}-e^{u}\right) \delta\left(v_{1}-w_{1}\right) \delta\left(v_{2}-w_{2}\right) d u d w_{1} d w_{2} \\
+ & \int_{0}^{\tau} e^{-r(\tau-\xi)} \int_{0}^{\infty} \int_{0}^{\infty} \int_{-\infty}^{\infty} f\left(\xi, x, v_{1}, v_{2}\right)\left[\frac{\partial U}{\partial \tau}-\mathcal{D}_{x, v_{1}, v_{2}} U\right] d u d w_{1} d w_{2} d \xi-f\left(\tau, x, v_{1}, v_{2}\right) \\
& =f\left(\tau, x, v_{1}, v_{2}\right)+0-f\left(\tau, x, v_{1}, v_{2}\right)=0
\end{aligned}
$$


Hence $C\left(\tau, x, v_{1}, v_{2}\right)$ satisfies the $\operatorname{PDE}(\mathrm{A} 1.1)$.

\section{Appendix 2. Proof of Proposition 5.1}

By use of equation (4.1) and the assumptions in (4.5), we note that

$$
\begin{aligned}
& \mathcal{F}\left\{\frac{\partial U}{\partial x}\left(\tau, x, v_{1}, v_{2}\right)\right\}=-i \eta \hat{U}\left(\tau, \eta, v_{1}, v_{2}\right), \quad \mathcal{F}\left\{\frac{\partial^{2} U}{\partial x^{2}}\left(\tau, x, v_{1}, v_{2}\right)\right\}=-\eta^{2} \hat{U}\left(\tau, \eta, v_{1}, v_{2}\right), \\
& \mathcal{F}\left\{\frac{\partial^{2} U}{\partial x \partial v_{1}}\left(\tau, x, v_{1}, v_{2}\right)\right\}=-i \eta \frac{\partial \hat{U}}{\partial v_{1}}\left(\tau, \eta, v_{1}, v_{2}\right), \quad \mathcal{F}\left\{\frac{\partial^{2} U}{\partial x \partial v_{2}}\left(\tau, x, v_{1}, v_{2}\right)\right\}=-i \eta \frac{\partial \hat{U}}{\partial v_{2}}\left(\tau, \eta, v_{1}, v_{2}\right), \\
& \mathcal{F}\left\{\frac{\partial U}{\partial v_{1}}\left(\tau, x, v_{1}, v_{2}\right)\right\}=\frac{\partial \hat{U}}{\partial v_{1}}\left(\tau, \eta, v_{1}, v_{2}\right), \quad \mathcal{F}\left\{\frac{\partial U}{\partial v_{2}}\left(\tau, x, v_{1}, v_{2}\right)\right\}=\frac{\partial \hat{U}}{\partial v_{2}}\left(\tau, \eta, v_{1}, v_{2}\right), \\
& \mathcal{F}\left\{\frac{\partial^{2} U}{\partial v_{1}^{2}}\left(\tau, x, v_{1}, v_{2}\right)\right\}=\frac{\partial^{2} \hat{U}}{\partial v_{1}^{2}}\left(\tau, \eta, v_{1}, v_{2}\right), \quad \mathcal{F}\left\{\frac{\partial^{2} U}{\partial v_{2}^{2}}\left(\tau, x, v_{1}, v_{2}\right)\right\}=\frac{\partial^{2} \hat{U}}{\partial v_{2}^{2}}\left(\tau, \eta, v_{1}, v_{2}\right), \\
& \mathcal{F}\left\{\frac{\partial U}{\partial \tau}\left(\tau, x, v_{1}, v_{2}\right)\right\}=\frac{\partial \hat{U}}{\partial \tau}\left(\tau, \eta, v_{1}, v_{2}\right) .
\end{aligned}
$$

Substituting all these expressions into equation (3.8) we obtain the PDE in Proposition 5.1.

The Fourier transform of the initial condition in equation (3.9) is simplified as follows:

$$
\begin{aligned}
\mathcal{F}\left\{U\left(0, x, v_{1}, v_{2}\right)\right\} & =\int_{-\infty}^{\infty} e^{i \eta x} U\left(0, x, v_{1}, v_{2}\right) d x \\
& =\int_{-\infty}^{\infty} e^{i \eta x} \delta\left(x-x_{0}\right) \delta\left(v_{1}-v_{1,0}\right) \delta\left(v_{2}-v_{2,0}\right) d x \\
& =e^{i \eta x_{0}} \delta\left(v_{1}-v_{1,0}\right) \delta\left(v_{2}-v_{2,0}\right),
\end{aligned}
$$

which is the result presented in equation (5.3) of Proposition 5.1. 


\section{Appendix 3. Proof of Proposition 6.1}

By applying equation (4.3) and the assumptions in (4.6) and (4.7) to the respective components of equation (5.1) we obtain

$$
\begin{aligned}
& \mathcal{L}\left\{v_{1} \hat{U}\left(\tau, \eta, v_{1}, v_{2}\right)\right\}=-\frac{\partial}{\partial s_{1}} \int_{0}^{\infty} \int_{0}^{\infty} e^{-s_{1} v_{1}-s_{2} v_{2}} \hat{U} d v_{1} d v_{2}=-\frac{\partial \tilde{U}}{\partial s_{1}}\left(\tau, \eta, s_{1}, s_{2}\right), \\
& \mathcal{L}\left\{v_{2} \hat{U}\left(\tau, \eta, v_{1}, v_{2}\right)\right\}=-\frac{\partial}{\partial s_{2}} \int_{0}^{\infty} \int_{0}^{\infty} e^{-s_{1} v_{1}-s_{2} v_{2}} \hat{U} d v_{1} d v_{2}=-\frac{\partial \tilde{U}}{\partial s_{2}}\left(\tau, \eta, s_{1}, s_{2}\right), \\
& \mathcal{L}\left\{\frac{\partial \hat{U}}{\partial v_{1}}\left(\tau, \eta, v_{1}, v_{2}\right)\right\}=\int_{0}^{\infty} \int_{0}^{\infty} e^{-s_{1} v_{1}-s_{2} v_{2}} \frac{\partial \hat{U}}{\partial v_{1}} d v_{1} d v_{2}=-\tilde{U}\left(\tau, \eta, 0, s_{2}\right)+s_{1} \tilde{U}\left(\tau, \eta, s_{1}, s_{2}\right), \\
& \mathcal{L}\left\{\frac{\partial \hat{U}}{\partial v_{2}}\left(\tau, \eta, v_{1}, v_{2}\right)\right\}=\int_{0}^{\infty} \int_{0}^{\infty} e^{-s_{1} v_{1}-s_{2} v_{2}} \frac{\partial \hat{U}}{\partial v_{2}} d v_{1} d v_{2}=-\tilde{U}\left(\tau, \eta, s_{1}, 0\right)+s_{2} \tilde{U}\left(\tau, \eta, s_{1}, s_{2}\right), \\
& \mathcal{L}\left\{v_{1} \frac{\partial \hat{U}}{\partial v_{1}}\left(\tau, \eta, v_{1}, v_{2}\right)\right\}=\int_{0}^{\infty} \int_{0}^{\infty} v_{1} e^{-s_{1} v_{1}-s_{2} v_{2}} \frac{\partial \hat{U}}{\partial v_{1}} d v_{1} d v_{2} \\
& =-\frac{\partial}{\partial s_{1}} \int_{0}^{\infty} \int_{0}^{\infty} e^{-s_{1} v_{1}-s_{2} v_{2}} \frac{\partial \hat{U}}{\partial v_{1}} d v_{1} d v_{2} \\
& =-\frac{\partial}{\partial s_{1}}\left[-\tilde{U}\left(\tau, \eta, 0, s_{2}\right)+s_{1} \tilde{U}\left(\tau, \eta, s_{1}, s_{2}\right)\right] \\
& =-\tilde{U}\left(\tau, \eta, s_{1}, s_{2}\right)-s_{1} \frac{\partial \tilde{U}}{\partial s_{1}}\left(\tau, \eta, s_{1}, s_{2}\right), \\
& \mathcal{L}\left\{v_{2} \frac{\partial \hat{U}}{\partial v_{2}}\left(\tau, \eta, v_{1}, v_{2}\right)\right\}=\int_{0}^{\infty} \int_{0}^{\infty} v_{2} e^{-s_{1} v_{1}-s_{2} v_{2}} \frac{\partial \hat{U}}{\partial v_{1}} d v_{1} d v_{2} \\
& =-\frac{\partial}{\partial s_{2}} \int_{0}^{\infty} \int_{0}^{\infty} e^{-s_{1} v_{1}-s_{2} v_{2}} \frac{\partial \hat{U}}{\partial v_{2}} d v_{1} d v_{2} \\
& =-\frac{\partial}{\partial s_{2}}\left[-\tilde{U}\left(\tau, \eta, s_{1}, 0\right)+s_{2} \tilde{U}\left(\tau, \eta, s_{1}, s_{2}\right)\right] \\
& =-\tilde{U}\left(\tau, \eta, s_{1}, s_{2}\right)-s_{2} \frac{\partial \tilde{U}}{\partial s_{2}}\left(\tau, \eta, s_{1}, s_{2}\right), \\
& \mathcal{L}\left\{v_{1} \frac{\partial^{2} \hat{U}}{\partial v_{1}^{2}}\left(\tau, \eta, v_{1}, v_{2}\right)\right\}=\tilde{U}\left(\tau, \eta, 0, s_{2}\right)-2 s_{1} \tilde{U}\left(\tau, \eta, s_{1}, s_{2}\right)-s_{1}^{2} \frac{\partial \tilde{U}}{\partial s_{1}}\left(\tau, \eta, s_{1}, s_{2}\right), \\
& \mathcal{L}\left\{v_{2} \frac{\partial^{2} \hat{U}}{\partial v_{2}^{2}}\left(\tau, \eta, v_{1}, v_{2}\right)\right\}=\tilde{U}\left(\tau, \eta, s_{1}, 0\right)-2 s_{2} \tilde{U}\left(\tau, \eta, s_{1}, s_{2}\right)-s_{2}^{2} \frac{\partial \tilde{U}}{\partial s_{2}}\left(\tau, \eta, s_{1}, s_{2}\right)
\end{aligned}
$$

Substituting these expressions into equation (5.1) and noting that $f_{1}\left(\tau, s_{2}\right)$ and $f_{2}\left(\tau, s_{1}\right)$ are terms involving the Laplace transforms of $\tilde{U}\left(\tau, \eta, 0, s_{2}\right)$ and $\tilde{U}\left(\tau, \eta, s_{1}, 0\right)$ we obtain the result in Proposition 6.1. Feller (1951) has demonstrated that assumptions like those in the first equation of (4.7) imply that

$$
\lim _{s_{1} \rightarrow \infty} \tilde{U}\left(\tau, \eta, s_{1}, s_{2}\right)=0 \quad \text { and } \quad \lim _{s_{2} \rightarrow \infty} \tilde{U}\left(\tau, \eta, s_{1}, s_{2}\right)=0
$$

which is equation (6.3) of Proposition 6.1. 


\section{Appendix 4. Proof of Proposition 7.1}

This appendix contains lengthy derivations for generating the solution of the partial differential equation system (6.1). Because of the nature of this PDE, we use the method of characteristics to find its solution. We break the appendix into three major parts where the first involves derivation of the general solution of the characteristic equations. The second part involves determination of the two functions, $f_{1}\left(\tau, S_{2}\right)$ and $f_{2}\left(\tau, S_{1}\right)$ appearing in equation (6.1). Once these two functions are determined, we then present the explicit form of $\tilde{U}\left(\tau, \eta, s_{1}, s_{2}\right)$ in the third part.

1. Solving the Characteristic equation in terms of $f_{1}\left(\tau, S_{2}\right)$ and $f_{2}\left(\tau, S_{1}\right)$ :

Here we attempt to solve equation (6.1) subject to the initial condition (6.2) by using the method of characteristics. Equation (6.1) can be re-expressed in characteristic form as

$$
\begin{aligned}
d \tau & =\frac{d s_{1}}{\frac{1}{2} \sigma_{1}^{2} s_{1}^{2}-\Theta_{1} s_{1}+\frac{1}{2} \Lambda}=\frac{d s_{2}}{\frac{1}{2} \sigma_{2}^{2} s_{2}^{2}-\Theta_{2} s_{2}+\frac{1}{2} \Lambda} \\
& =\frac{d \tilde{U}}{\left\{\left(\Phi_{1}-\sigma_{1}^{2}\right) s_{1}-i \eta(r-q)+\left(\Phi_{2}-\sigma_{2}^{2}\right) s_{2}+\Theta_{1}+\Theta_{2}\right\} \tilde{U}+f_{1}\left(\tau, s_{2}\right)+f_{2}\left(\tau, s_{1}\right)}
\end{aligned}
$$




\section{Simplifying the first characteristic pair}

By adopting the method of characteristics, we solve the first pair of equation (A4.1) by integration to obtain

$$
\int d \tau=\frac{2}{\sigma_{1}^{2}} \int \frac{d s_{1}}{s_{1}^{2}-\frac{2 \Theta_{1}}{\sigma_{1}^{2}} s_{1}+\frac{\Lambda}{\sigma_{1}^{2}}} .
$$

By factorising the RHS of the above equation we obtain

$$
\int d \tau=\frac{1}{\Omega_{1}} \int\left(\frac{1}{s_{1}-\left(\frac{\Theta_{1}+\Omega_{1}}{\sigma_{1}^{2}}\right)}-\frac{1}{s_{1}-\left(\frac{\Theta_{1}-\Omega_{1}}{\sigma_{1}^{2}}\right)}\right) d s_{1} .
$$

where we set

$$
\Omega_{1}=\sqrt{\Theta_{1}^{2}-\Lambda(\eta) \sigma_{1}^{2}} .
$$

Equation (A4.2) implies that

$$
\tau+c_{1}=\frac{1}{\Omega_{1}} \int\left(\frac{1}{s_{1}-\left(\frac{\Theta_{1}+\Omega_{1}}{\sigma_{1}^{2}}\right)}-\frac{1}{s_{1}-\left(\frac{\Theta_{1}-\Omega_{1}}{\sigma_{1}^{2}}\right)}\right) d s_{1},
$$

where $c_{1}$ is an integration constant ${ }^{9}$. Integrating the RHS yields

$$
\Omega_{1} \tau+c_{2}=\ln \left(\frac{\sigma_{1}^{2} s_{1}-\Theta_{1}-\Omega_{1}}{\sigma_{1}^{2} s_{1}-\Theta_{1}+\Omega_{1}}\right)
$$

which implies that

$$
e^{\Omega_{1} \tau} e^{c_{2}}=\frac{\sigma_{1}^{2} s_{1}-\Theta_{1}-\Omega_{1}}{\sigma_{1}^{2} s_{1}-\Theta_{1}+\Omega_{1}}
$$

hence

$$
e^{c_{2}}=\frac{\left(\sigma_{1}^{2} s_{1}-\Theta_{1}-\Omega_{1}\right) e^{-\Omega_{1} \tau}}{\sigma_{1}^{2} s_{1}-\Theta_{1}+\Omega_{1}} .
$$

The exponent of an integration constant is another constant, so that equation (A4.5) can be represented as

$$
c_{3}=\frac{\left(\sigma_{1}^{2} s_{1}-\Theta_{1}-\Omega_{1}\right) e^{-\Omega_{1} \tau}}{\sigma_{1}^{2} s_{1}-\Theta_{1}+\Omega_{1}} .
$$

Now, given equation (A4.6), we can obtain an expression for $s_{1}$ by making it the subject of the formula such that

$$
c_{3}\left(\sigma_{1}^{2} s_{1}-\Theta_{1}+\Omega_{1}\right)=\left(\sigma_{1}^{2} s_{1}-\Theta_{1}-\Omega_{1}\right) e^{-\Omega_{1} \tau},
$$

that is

$$
c_{3} \sigma_{1}^{2} s_{1}-\sigma_{1}^{2} s_{1} e^{-\Omega_{1} \tau}=\left(\Theta_{1}-\Omega_{1}\right) c_{3}-\left(\Theta_{1}+\Omega_{1}\right) e^{-\Omega_{1} \tau},
$$

\footnotetext{
${ }^{9}$ In what follows we use $c_{j}$ and $d_{j}, j=1,2,3$ to denote integration constants.
} 
which becomes

$$
\sigma_{1}^{2}\left(c_{3}-e^{-\Omega_{1} \tau}\right) s_{1}=\Theta_{1}\left(c_{3}-e^{-\Omega_{1} \tau}\right)-\Omega_{1}\left(c_{3}+e^{-\Omega_{1} \tau}+e^{-\Omega_{1} \tau}-e^{-\Omega_{1} \tau}\right),
$$

which then implies that

$$
s_{1}=\frac{\Theta_{1}-\Omega_{1}}{\sigma_{1}^{2}}-\frac{2 \Omega_{1} e^{-\Omega_{1} \tau}}{\sigma_{1}^{2}\left(c_{3}-e^{-\Omega_{1} \tau}\right)} .
$$

\section{Solving the second characteristic pair}

The characteristic equation of the second pair can be represented as

$$
\int d \tau=\frac{2}{\sigma_{2}^{2}} \int \frac{d s_{2}}{s_{2}^{2}-\frac{2 \Theta_{2}}{\sigma_{2}^{2}} s_{2}+\frac{\Lambda}{\sigma_{2}^{2}}} .
$$

By factorising the RHS we obtain

$$
\int d \tau=\frac{1}{\Omega_{2}} \int\left(\frac{1}{s_{2}-\left(\frac{\Theta_{2}+\Omega_{2}}{\sigma_{2}^{2}}\right)}-\frac{1}{s_{2}-\left(\frac{\Theta_{2}-\Omega_{2}}{\sigma_{2}^{2}}\right)}\right) d s_{2},
$$

where we set

$$
\Omega_{2}=\sqrt{\Theta_{2}^{2}-\Lambda(\eta) \sigma_{2}^{2}}
$$

The above equation simplifies to

$$
\tau+d_{1}=\frac{1}{\Omega_{2}} \int\left(\frac{1}{s_{2}-\left(\frac{\Theta_{2}+\Omega_{2}}{\sigma_{2}^{2}}\right)}-\frac{1}{s_{2}-\left(\frac{\Theta_{2}-\Omega_{2}}{\sigma_{2}^{2}}\right)}\right) d s_{2} .
$$

Solving the RHS yields

$$
\Omega_{2} \tau+d_{2}=\ln \left(\frac{\sigma_{2}^{2} s_{2}-\Theta_{2}-\Omega_{2}}{\sigma_{2}^{2} s_{2}-\Theta_{2}+\Omega_{2}}\right)
$$

which implies that

$$
e^{\Omega_{2} \tau} e^{d_{2}}=\frac{\sigma_{2}^{2} s_{2}-\Theta_{2}-\Omega_{2}}{\sigma_{2}^{2} s_{2}-\Theta_{2}+\Omega_{2}}
$$

hence

$$
e^{d_{2}}=\frac{\left(\sigma_{2}^{2} s_{2}-\Theta_{2}-\Omega_{2}\right) e^{-\Omega_{2} \tau}}{\sigma_{2}^{2} s_{2}-\Theta_{2}+\Omega_{2}}
$$

which can be written as

$$
d_{3}=\frac{\left(\sigma_{2}^{2} s_{2}-\Theta_{2}-\Omega_{2}\right) e^{-\Omega_{2} \tau}}{\sigma_{2}^{2} s_{2}-\Theta_{2}+\Omega_{2}}
$$

Given equation (A4.11), we can show that

$$
s_{2}=\frac{\Theta_{2}-\Omega_{2}}{\sigma_{2}^{2}}-\frac{2 \Omega_{2} e^{-\Omega_{2} \tau}}{\sigma_{2}^{2}\left(d_{3}-e^{-\Omega_{2} \tau}\right)} .
$$




\section{Solving the third characteristic pair}

We now turn to the last pair in equation (A4.1) which we represent as

$$
\begin{gathered}
\frac{d \tilde{U}}{d \tau}+\left\{\left(\sigma_{1}^{2}-\Phi_{1}\right) s_{1}+\right. \\
\left.\left(\sigma_{2}^{2}-\Phi_{2}\right) s_{2}+i \eta(r-q)-\Theta_{1}-\Theta_{2}\right\} \tilde{U} \\
=f_{1}\left(\tau, s_{2}\right)+f_{2}\left(\tau, s_{1}\right)
\end{gathered}
$$

where $s_{1}$ and $s_{2}$ are given by equations (A4.7) and (A4.12) respectively. The integrating factor of equation (A4.13) is

$$
R(\tau)=\exp \left(\int\left\{\left(\sigma_{1}^{2}-\Phi_{1}\right) s_{1}+\left(\sigma_{2}^{2}-\Phi_{2}\right) s_{2}+i \eta(r-q)-\Theta_{1}-\Theta_{2}\right\} d \tau\right) .
$$

The integral inside the exponent can be simplified as

$$
\begin{aligned}
& \int\left(\left(\sigma_{1}^{2}-\Phi_{1}\right)\left[\frac{\Theta_{1}-\Omega_{1}}{\sigma_{1}^{2}}-\frac{2 \Omega_{1} e^{-\Omega_{1} \tau}}{\sigma_{1}^{2}\left(c_{3}-e^{-\Omega_{1} \tau}\right)}\right]+\left(\sigma_{2}^{2}-\Phi_{2}\right)\left[\frac{\Theta_{2}-\Omega_{2}}{\sigma_{2}^{2}}-\frac{2 \Omega_{2} e^{-\Omega_{2} \tau}}{\sigma_{2}^{2}\left(d_{3}-e^{-\Omega_{2} \tau}\right)}\right]\right. \\
& \left.+i \eta(r-q)-\Theta_{1}-\Theta_{2}\right) d \tau \\
& =\left\{\frac{\left(\sigma_{1}^{2}-\Phi_{1}\right)\left(\Theta_{1}-\Omega_{1}\right)}{\sigma_{1}^{2}}+\frac{\left(\sigma_{2}^{2}-\Phi_{2}\right)\left(\Theta_{2}-\Omega_{2}\right)}{\sigma_{2}^{2}}+i \eta(r-q)-\Theta_{1}-\Theta_{2}\right\} \tau \\
& -\left(\sigma_{1}^{2}-\Phi_{1}\right) \int \frac{2 \Omega_{1} e^{-\Omega_{1} \tau}}{\sigma_{1}^{2}\left(c_{3}-e^{-\Omega_{1} \tau}\right)} d \tau-\left(\sigma_{2}^{2}-\Phi_{2}\right) \int \frac{2 \Omega_{2} e^{-\Omega_{2} \tau}}{\sigma_{2}^{2}\left(d_{3}-e^{-\Omega_{2} \tau}\right)} d \tau \\
& =\left\{\frac{\left(\sigma_{1}^{2}-\Phi_{1}\right)\left(\Theta_{1}-\Omega_{1}\right)}{\sigma_{1}^{2}}+\frac{\left(\sigma_{2}^{2}-\Phi_{2}\right)\left(\Theta_{2}-\Omega_{2}\right)}{\sigma_{2}^{2}}+i \eta(r-q)-\Theta_{1}-\Theta_{2}\right\} \tau \\
& -\frac{2 \Omega_{1}\left(\sigma_{1}^{2}-\Phi_{1}\right)}{\sigma_{1}^{2}} \int \frac{e^{-\Omega_{1} \tau}}{c_{3}-e^{-\Omega_{1} \tau}} d \tau-\frac{2 \Omega_{2}\left(\sigma_{2}^{2}-\Phi_{2}\right)}{\sigma_{2}^{2}} \int \frac{e^{-\Omega_{2} \tau}}{d_{3}-e^{-\Omega_{2} \tau}} d \tau .
\end{aligned}
$$

In the first integral on the last line set $u_{1}=c_{3}-e^{-\Omega_{1} \tau}$, and in the second integral set $u_{2}=d_{3}-e^{-\Omega_{2} \tau}$. The two integral components of (A4.15) then simplify to

$$
\begin{aligned}
\int \frac{e^{-\Omega_{1} \tau}}{c_{3}-e^{-\Omega_{1} \tau}} d \tau & =\frac{1}{\Omega_{1}} \int \frac{d u_{1}}{u_{1}}=\frac{1}{\Omega_{1}} \ln \left|u_{1}\right| \\
& =\frac{1}{\Omega_{1}} \ln \left|c_{3}-e^{-\Omega_{1} \tau}\right|,
\end{aligned}
$$

and

$$
\int \frac{e^{-\Omega_{2} \tau}}{d_{3}-e^{-\Omega_{2} \tau}} d \tau=\frac{1}{\Omega_{2}} \ln \left|d_{3}-e^{-\Omega_{2} \tau}\right|
$$


Thus the integrating factor of (A4.13) can be represented as

$$
\begin{aligned}
& R(\tau)=\left.\left|\frac{1}{c_{3}-e^{-\Omega_{1} \tau}}\right|\right|^{\frac{2}{\sigma_{1}^{2}}\left(\sigma_{1}^{2}-\Phi_{1}\right)}\left|\frac{1}{d_{3}-e^{-\Omega_{2} \tau}}\right| \frac{2}{\sigma_{2}^{2}}\left(\sigma_{2}^{2}-\Phi_{2}\right) \\
& \quad \times \exp \left\{\left[\frac{\left(\sigma_{1}^{2}-\Phi_{1}\right)\left(\Theta_{1}-\Omega_{1}\right)}{\sigma_{1}^{2}}+\frac{\left(\sigma_{2}^{2}-\Phi_{2}\right)\left(\Theta_{2}-\Omega_{2}\right)}{\sigma_{2}^{2}}+i \eta(r-q)-\Theta_{1}-\Theta_{2}\right] \tau\right\} .
\end{aligned}
$$

Now that we have the integrating factor of equation (A4.13), we can solve this equation by writing it as

$$
\frac{d}{d \tau}\left(R(\tau) \tilde{U}\left(\tau, \eta, s_{1}, s_{2}\right)\right)=R(\tau)\left[f_{1}\left(\tau, s_{2}\right)+f_{2}\left(\tau, s_{1}\right)\right]
$$

Integrating the above system yields

$$
R(\tau) \tilde{U}\left(\tau, \eta, s_{1}, s_{2}\right)=\int_{0}^{\tau} R(t)\left[f_{1}\left(t, s_{2}\right)+f_{2}\left(t, s_{1}\right)\right] d t+c_{4}
$$

which implies that

$$
\tilde{U}\left(\tau, \eta, s_{1}, s_{2}\right)=\frac{1}{R(\tau)}\left\{\int_{0}^{\tau} R(t)\left[f_{1}\left(t, s_{2}\right)+f_{2}\left(t, s_{1}\right)\right] d t+c_{4}\right\} .
$$

The above equation can be explicitly represented as

$$
\begin{aligned}
& \tilde{U}\left(\tau, \eta, s_{1}, s_{2}\right)=\exp \left\{\left[\frac{\left(\Phi_{1}-\sigma_{1}^{2}\right)\left(\Theta_{1}-\Omega_{1}\right)}{\sigma_{1}^{2}}+\frac{\left(\Phi_{2}-\sigma_{2}^{2}\right)\left(\Theta_{2}-\Omega_{2}\right)}{\sigma_{2}^{2}}-i \eta(r-q)+\Theta_{1}+\Theta_{2}\right] \tau\right\} \\
& \times\left|c_{3}-e^{-\Omega_{1} \tau}\right|^{\frac{2}{\sigma_{1}^{2}}\left(\sigma_{1}^{2}-\Phi_{1}\right)}\left|d_{3}-e^{-\Omega_{2} \tau}\right|^{\frac{2}{\sigma_{2}^{2}}\left(\sigma_{2}^{2}-\Phi_{2}\right)} \\
& \times\left\{\int_{0}^{\tau}\left[f_{1}\left(t, s_{2}\right)+f_{2}\left(t, s_{1}\right)\right]\right. \\
& \times \exp \left(\left[\frac{\left(\sigma_{1}^{2}-\Phi_{1}\right)\left(\Theta_{1}-\Omega_{1}\right)}{\sigma_{1}^{2}}+\frac{\left(\sigma_{2}^{2}-\Phi_{2}\right)\left(\Theta_{2}-\Omega_{2}\right)}{\sigma_{2}^{2}}+i \eta(r-q)-\Theta_{1}-\Theta_{2}\right] t\right) \\
& \left.\times\left|\frac{1}{c_{3}-e^{-\Omega_{1} t}}\right|^{\frac{2}{\sigma_{1}^{2}}\left(\sigma_{1}^{2}-\Phi_{1}\right)}\left|\frac{1}{d_{3}-e^{-\Omega_{2} t}}\right|^{\frac{2}{\sigma_{2}^{2}}\left(\sigma_{2}^{2}-\Phi_{2}\right)} d t+c_{4}\right\} \text {. }
\end{aligned}
$$

Here, $c_{4}$ is a constant of integration whose value is determined by use of the initial condition, that is when $\tau=0$.

\section{$\underline{\text { Determining the integration constant, } \mathbf{c}_{4}}$.}

The constant, $c_{4}$, is a function of two constants namely $c_{3}$ and $d_{3}$ which are given by (A4.6) and (A4.11) respectively. By letting $c_{4}=A\left(c_{3}, d_{3}\right)$ at $\tau=0$, it can be readily 
shown that equation (A4.20) becomes

$$
\begin{aligned}
& \tilde{U}\left(0, \eta, s_{1}, s_{2}\right)=A\left(c_{3}, d_{3}\right) \\
& \quad \times\left|\frac{\sigma_{1}^{2} s_{1}-\Theta_{1}-\Omega_{1}}{\sigma_{1}^{2} s_{1}-\Theta_{1}+\Omega_{1}}-1\right|^{\frac{2}{\sigma_{1}^{2}}\left(\sigma_{1}^{2}-\Phi_{1}\right)}\left|\frac{\sigma_{2}^{2} s_{2}-\Theta_{2}-\Omega_{2}}{\sigma_{2}^{2} s_{2}-\Theta_{2}+\Omega_{2}}-1\right|^{\frac{2}{\sigma_{2}^{2}}\left(\sigma_{2}^{2}-\Phi_{2}\right)} .
\end{aligned}
$$

By substituting the values of $s_{1}$ and $s_{2}$ from equations (A4.7) and (A4.12) at $\tau=0$ and making $A\left(c_{3}, d_{3}\right)$ the subject of formula we obtain

$$
\begin{aligned}
& A\left(c_{3}, d_{3}\right)=\left|c_{3}-1\right|^{-\frac{2}{\sigma_{1}^{2}}\left(\sigma_{1}^{2}-\Phi_{1}\right)}\left|d_{3}-1\right|^{-\frac{2}{\sigma_{2}^{2}}\left(\sigma_{2}^{2}-\Phi_{2}\right)} \\
& \quad \times \tilde{U}\left(0, \eta, \frac{\Theta_{1}-\Omega_{1}}{\sigma_{1}^{2}}-\frac{2 \Omega_{1}}{\sigma_{1}^{2}\left(c_{3}-1\right)}, \frac{\Theta_{2}-\Omega_{2}}{\sigma_{2}^{2}}-\frac{2 \Omega_{2}}{\sigma_{2}^{2}\left(d_{3}-1\right)}\right) .
\end{aligned}
$$

Having determined $A\left(c_{3}, d_{3}\right)$, the expression involving the constant term in equation (A4.20) can be written as

$$
\begin{aligned}
&\left|c_{3}-e^{-\Omega_{1} \tau}\right|^{\frac{2}{\sigma_{1}^{2}}\left(\sigma_{1}^{2}-\Phi_{1}\right)}\left|d_{3}-e^{-\Omega_{2} \tau}\right|^{\frac{2}{\sigma_{2}^{2}}\left(\sigma_{2}^{2}-\Phi_{2}\right)} A\left(c_{3}, d_{3}\right) \\
&=\left|\frac{2 \Omega_{1} e^{-\Omega_{1} \tau}}{\left(\sigma_{1}^{2} s_{1}-\Theta_{1}+\Omega_{1}\right)\left(1-e^{-\Omega_{1} \tau}\right)+2 \Omega_{1} e^{-\Omega_{1} \tau}}\right| \frac{2 \Omega_{2} e^{-\Omega_{2} \tau}}{\sigma_{1}^{2}\left(\sigma_{1}^{2}-\Phi_{1}\right)} \\
& \times\left|\frac{2 \Omega_{2} e^{-\Omega_{2} \tau}}{\left(\sigma_{2}^{2} s_{2}-\Theta_{2}+\Omega_{2}\right)\left(1-e^{-\Omega_{2} \tau}\right)+2}\right| \frac{2}{\sigma_{2}^{2}\left(\sigma_{2}^{2}-\Phi_{2}\right)} \\
& \times \tilde{U}\left(0, \eta, \frac{\Theta_{1}-\Omega_{1}}{\sigma_{1}^{2}}-\frac{2 \Omega_{1}}{\sigma_{1}^{2}\left(c_{3}-1\right)}, \frac{\Theta_{2}-\Omega_{2}}{\sigma_{2}^{2}}-\frac{2 \Omega_{2}}{\sigma_{2}^{2}\left(d_{3}-1\right)}\right) .
\end{aligned}
$$

With the knowledge of the two constants, $c_{3}$ and $d_{3}$ as in equations (A4.6) and (A4.11), it can be shown that the expressions occurring in the arguments of $\tilde{U}$ in (A4.23) can be expressed as

$$
\frac{2 \Omega_{1}}{\sigma_{1}^{2}\left(c_{3}-1\right)}=\frac{2 \Omega_{1}\left(\sigma_{1}^{2} s_{1}-\Theta_{1}+\Omega_{1}\right)}{\sigma_{1}^{2}\left[\left(\sigma_{1}^{2} s_{1}-\Theta_{1}+\Omega_{1}\right)\left(e^{-\Omega_{1} \tau}-1\right)-2 \Omega_{1} e^{-\Omega_{1} \tau}\right]},
$$

and

$$
\frac{2 \Omega_{2}}{\sigma_{2}^{2}\left(d_{3}-1\right)}=\frac{2 \Omega_{2}\left(\sigma_{2}^{2} s_{2}-\Theta_{2}+\Omega_{2}\right)}{\sigma_{2}^{2}\left[\left(\sigma_{2}^{2} s_{2}-\Theta_{2}+\Omega_{2}\right)\left(e^{-\Omega_{2} \tau}-1\right)-2 \Omega_{2} e^{-\Omega_{2} \tau}\right]}
$$


Also by use of equations (A4.6) and (A4.11), for $0 \leq t \leq \tau$ we have

$$
\begin{aligned}
& \left|\frac{c_{3}-e^{-\Omega_{1} \tau}}{c_{3}-e^{-\Omega_{1} t}}\right|^{\frac{2}{\sigma_{1}^{2}}\left(\sigma_{1}^{2}-\Phi_{1}\right)}\left|\frac{d_{3}-e^{-\Omega_{2} \tau}}{d_{3}-e^{-\Omega_{2} t}}\right|^{\frac{2}{\sigma_{2}^{2}}\left(\sigma_{2}^{2}-\Phi_{2}\right)} \\
& =\left.\left|\frac{2 \Omega_{1} e^{-\Omega_{1} \tau}}{\left(\sigma_{1}^{2} s_{1}-\Theta_{1}+\Omega_{1}\right)\left(e^{-\Omega_{1} t}-e^{-\Omega_{1} \tau}\right)+2 \Omega_{1} e^{-\Omega_{1} \tau}}\right|\right|^{\frac{2}{\sigma_{1}^{2}}\left(\sigma_{1}^{2}-\Phi_{1}\right)} \\
& \times\left|\frac{2 \Omega_{2} e^{-\Omega_{2} \tau}}{\left(\sigma_{2}^{2} s_{2}-\Theta_{2}+\Omega_{2}\right)\left(e^{-\Omega_{2} t}-e^{-\Omega_{2} \tau}\right)+2 \Omega_{2} e^{-\Omega_{2} \tau}}\right|
\end{aligned}
$$

and it turns out that all real arguments in $|\cdot|$ are all positive. Substituting (A4.23) and (A4.26) into (A4.20) we obtain the expression for the transform as

$$
\begin{aligned}
& \tilde{U}\left(\tau, \eta, s_{1}, s_{2}\right)=\exp \left\{\left[\frac{\left(\Phi_{1}-\sigma_{1}^{2}\right)\left(\Theta_{1}-\Omega_{1}\right)}{\sigma_{1}^{2}}+\frac{\left(\Phi_{2}-\sigma_{2}^{2}\right)\left(\Theta_{2}-\Omega_{2}\right)}{\sigma_{2}^{2}}-i \eta(r-q)+\Theta_{1}+\Theta_{2}\right] \tau\right\} \\
& \times\left(\frac{2 \Omega_{1} e^{-\Omega_{1} \tau}}{\left(\sigma_{1}^{2} s_{1}-\Theta_{1}+\Omega_{1}\right)\left(1-e^{-\Omega_{1} \tau}\right)+2 \Omega_{1} e^{-\Omega_{1} \tau}}\right)^{\frac{2}{\sigma_{1}^{2}\left(\sigma_{1}^{2}-\Phi_{1}\right)}} \\
& \times\left(\frac{2 \Omega_{2} e^{-\Omega_{2} \tau}}{\left(\sigma_{2}^{2} s_{2}-\Theta_{2}+\Omega_{2}\right)\left(1-e^{-\Omega_{2} \tau}\right)+2 \Omega_{2} e^{-\Omega_{2} \tau}}\right)^{\frac{2}{\sigma_{2}^{2}\left(\sigma_{2}^{2}-\Phi_{2}\right)}} \\
& \times \tilde{U}\left(0, \eta, \frac{\Theta_{1}-\Omega_{1}}{\sigma_{1}^{2}}-\frac{2 \Omega_{1}\left(\sigma_{1}^{2} s_{1}-\Theta_{1}+\Omega_{1}\right)}{\sigma_{1}^{2}\left[\left(\sigma_{1}^{2} s_{1}-\Theta_{1}+\Omega_{1}\right)\left(e^{-\Omega_{1} \tau}-1\right)-2 \Omega_{1} e^{-\Omega_{1} \tau}\right]},\right. \\
& \left.\frac{\Theta_{2}-\Omega_{2}}{\sigma_{2}^{2}}-\frac{2 \Omega_{2}\left(\sigma_{2}^{2} s_{2}-\Theta_{2}+\Omega_{2}\right)}{\sigma_{2}^{2}\left[\left(\sigma_{2}^{2} s_{2}-\Theta_{2}+\Omega_{2}\right)\left(e^{-\Omega_{2} \tau}-1\right)-2 \Omega_{2} e^{-\Omega_{2} \tau}\right]}\right) \\
& +\int_{0}^{\tau}\left[f_{1}\left(t, s_{2}\right)+f_{2}\left(t, s_{1}\right)\right] \\
& \times \exp \left\{\left[\frac{\left(\Phi_{1}-\sigma_{1}^{2}\right)\left(\Theta_{1}-\Omega_{1}\right)}{\sigma_{1}^{2}}+\frac{\left(\Phi_{2}-\sigma_{2}^{2}\right)\left(\Theta_{2}-\Omega_{2}\right)}{\sigma_{2}^{2}}-i \eta(r-q)+\Theta_{1}+\Theta_{2}\right](\tau-t)\right\} \\
& \times\left(\frac{2 \Omega_{1} e^{-\Omega_{1} \tau}}{\left(\sigma_{1}^{2} s_{1}-\Theta_{1}+\Omega_{1}\right)\left(e^{-\Omega_{1} t}-e^{-\Omega_{1} \tau}\right)+2 \Omega_{1} e^{-\Omega_{1} \tau}}\right)^{\frac{2}{\sigma_{1}^{2}\left(\sigma_{1}^{2}-\Phi_{1}\right)}} \\
& \times\left(\frac{2 \Omega_{2} e^{-\Omega_{2} \tau}}{\left(\sigma_{2}^{2} s_{2}-\Theta_{2}+\Omega_{2}\right)\left(e^{-\Omega_{2} t}-e^{-\Omega_{2} \tau}\right)+2 \Omega_{2} e^{-\Omega_{2} \tau}}\right)^{\frac{2}{\sigma_{2}^{2}}\left(\sigma_{2}^{2}-\Phi_{2}\right)} d t .
\end{aligned}
$$

This expression for the transform still involves the yet unknown functions $f_{1}\left(t, s_{2}\right)$ and $f_{2}\left(t, s_{1}\right)$. We next discuss how to obtain these functions.

\section{Determining the functional forms of $\mathbf{f}_{\mathbf{1}}\left(\tau, \mathbf{s}_{\mathbf{2}}\right)$ and $\mathbf{f}_{\mathbf{2}}\left(\tau, \mathbf{s}_{\mathbf{1}}\right)$ :}

The task of finding the functional forms of $f_{1}\left(\tau, s_{2}\right)$ and $f_{2}\left(\tau, s_{1}\right)$ is accomplished by

using the conditions in equation (6.3). We first tackle the $f_{1}\left(\tau, s_{2}\right)$ component. As $s_{1} \rightarrow \infty$ and making use of l'Hôpital's rule, equation (A4.27) simplifies to 


$$
\begin{aligned}
-\tilde{U} & \left(0, \eta, \frac{\Theta_{1}-\Omega_{1}}{\sigma_{1}^{2}}-\frac{2 \Omega_{1}}{\sigma_{1}^{2}\left(e^{-\Omega_{1} \tau}-1\right)}, \frac{\Theta_{2}-\Omega_{2}}{\sigma_{2}^{2}}-\frac{2 \Omega_{2}\left(\sigma_{2}^{2} s_{2}-\Theta_{2}+\Omega_{2}\right)}{\sigma_{2}^{2}\left[\left(\sigma_{2}^{2} s_{2}-\Theta_{2}+\Omega_{2}\right)\left(e^{-\Omega_{2} \tau}-1\right)-2 \Omega_{2} e^{-\Omega_{2} \tau}\right]}\right) \\
= & \int_{0}^{\tau} f_{1}\left(t, s_{2}\right) \exp \left\{-\left[\frac{\left(\Phi_{1}-\sigma_{1}^{2}\right)\left(\Theta_{1}-\Omega_{1}\right)}{\sigma_{1}^{2}}+\frac{\left(\Phi_{2}-\sigma_{2}^{2}\right)\left(\Theta_{2}-\Omega_{2}\right)}{\sigma_{2}^{2}}-i \eta(r-q)+\Theta_{1}+\Theta_{2}\right] t\right\} \\
& \times\left(\frac{1-e^{-\Omega_{1} \tau}}{e^{-\Omega_{1} t}-e^{-\Omega_{1} \tau}}\right)^{\frac{2}{\sigma_{1}^{2}\left(\sigma_{1}^{2}-\Phi_{1}\right)}}\left(\frac{\left(\sigma_{2}^{2} s_{2}-\theta_{2}+\Omega_{2}\right)\left(1-e^{-\Omega_{2} \tau}\right)+2 \Omega_{2} e^{-\Omega_{2} \tau}}{\left(\sigma_{2}^{2} s_{2}-\theta_{2}+\Omega_{2}\right)\left(e^{-\Omega_{2} t}-e^{-\Omega_{2} \tau}\right)+2 \Omega_{2} e^{-\Omega_{2} \tau}}\right)^{\frac{2}{\sigma_{2}^{2}}\left(\sigma_{2}^{2}-\Phi_{2}\right)} d t .
\end{aligned}
$$

Now let

$$
\begin{aligned}
& \zeta_{1}^{-1}=1-e^{-\Omega_{1} t}, \quad \zeta_{2}^{-1}=1-e^{-\Omega_{2} t}, \\
& z_{1}^{-1}=1-e^{-\Omega_{1} \tau}, \quad z_{2}^{-1}=1-e^{-\Omega_{2} \tau}
\end{aligned}
$$

We substitute these arguments into equation (A4.28) and defining the function

$$
\begin{aligned}
g_{1}\left(\zeta_{1}\right) & =f_{1}\left(t, s_{2}\right) \exp \left\{-\left[\frac{\left(\Phi_{1}-\sigma_{1}^{2}\right)\left(\Theta_{1}-\Omega_{1}\right)}{\sigma_{1}^{2}}+\frac{\left(\Phi_{2}-\sigma_{2}^{2}\right)\left(\Theta_{2}-\Omega_{2}\right)}{\sigma_{2}^{2}}-i \eta(r-q)+\Theta_{1}+\Theta_{2}\right] t\right\} \\
& \times\left(\frac{\zeta_{2}\left[\left(\sigma_{2}^{2} s_{2}-\Theta_{2}+\Omega_{2}\right)+2 \Omega_{2}\left(z_{2}-1\right)\right]}{\left(\sigma_{2}^{2} s_{2}-\Theta_{2}+\Omega_{2}\right)\left(\zeta_{2}-z_{2}\right)+2 \Omega_{2} \zeta_{2}\left(z_{2}-1\right)}\right)^{\frac{2}{\sigma_{2}^{2}}\left(\sigma_{2}^{2}-\Phi_{2}\right)} \frac{\frac{2}{\sigma_{1}^{2}}\left(\sigma_{1}^{2}-\Phi_{1}\right)}{\zeta_{1}\left(\zeta_{1}-1\right)} .
\end{aligned}
$$

which constitutes the terms inside the integrand of equation (A4.28) after factoring the substitutions in equations (A4.29) and (A4.30). Equation (A4.28) becomes after rearranging

$$
\begin{aligned}
\int_{z_{1}}^{\infty} g_{1}\left(\zeta_{1}\right) & \left(\zeta_{1}-z_{1}\right)^{\frac{2}{\sigma_{1}^{2}}\left(\Phi_{1}-\sigma_{1}^{2}\right)} d \zeta_{1} \\
& =-\Omega_{1} \tilde{U}\left(0, \eta, \frac{\Theta_{1}-\Omega_{1}}{\sigma_{1}^{2}}+\frac{2 \Omega_{1} z_{1}}{\sigma_{1}^{2}}, \frac{\Theta_{2}-\Omega_{2}}{\sigma_{2}^{2}}+\frac{\left(\sigma_{2}^{2} s_{2}-\Theta_{2}+\Omega_{2}\right) 2 \Omega_{2} z_{2}}{\left[\sigma_{2}^{2} s_{2}-\Theta_{2}+\Omega_{2}+2 \Omega_{2}\left(z_{2}-1\right)\right] \sigma_{2}^{2}}\right) .
\end{aligned}
$$

We can obtain another expression for

$$
\tilde{U}\left(0, \eta, \frac{\Theta_{1}-\Omega_{1}}{\sigma_{1}^{2}}+\frac{2 \Omega_{1} z_{1}}{\sigma_{1}^{2}}, \frac{\Theta_{2}-\Omega_{2}}{\sigma_{2}^{2}}+\frac{\left(\sigma_{2}^{2} s_{2}-\Theta_{2}+\Omega_{2}\right) 2 \Omega_{2} z_{2}}{\left[\sigma_{2}^{2} s_{2}-\Theta_{2}+\Omega_{2}+2 \Omega_{2}\left(z_{2}-1\right)\right] \sigma_{2}^{2}}\right)
$$

appearing in equation (A4.32) from the definition of the Laplace Transform. From Definition 4.3 we can write the Laplace transform on the RHS of equation (A4.32) as

$$
\begin{aligned}
& \tilde{U}\left(0, \eta, \frac{\Theta_{1}-\Omega_{1}}{\sigma_{1}^{2}}+\frac{2 \Omega_{1} z_{1}}{\sigma_{1}^{2}}, \frac{\Theta_{2}-\Omega_{2}}{\sigma_{2}^{2}}+\frac{\left(\sigma_{2}^{2} s_{2}-\Theta_{2}+\Omega_{2}\right) 2 \Omega_{2} z_{2}}{\left[\sigma_{2}^{2} s_{2}-\Theta_{2}+\Omega_{2}+2 \Omega_{2}\left(z_{2}-1\right)\right] \sigma_{2}^{2}}\right) \\
& =\int_{0}^{\infty} \int_{0}^{\infty} \exp \left\{-\left[\frac{\Theta_{1}-\Omega_{1}}{\sigma_{1}^{2}}+\frac{2 \Omega_{1} z_{1}}{\sigma_{1}^{2}}\right] v_{1}-\left[\frac{\Theta_{2}-\Omega_{2}}{\sigma_{2}^{2}}+\frac{\left(\sigma_{2}^{2} s_{2}-\Theta_{2}+\Omega_{2}\right) 2 \Omega_{2} z_{2}}{\left[\sigma_{2}^{2} s_{2}-\Theta_{2}+\Omega_{2}+2 \Omega_{2}\left(z_{2}-1\right)\right] \sigma_{2}^{2}}\right] v_{2}\right\} \\
& \times \hat{U}\left(0, \eta, v_{1}, v_{2}\right) d v_{1} d v_{2} .
\end{aligned}
$$


For convenience, we introduce a gamma function such that ${ }^{10}$

$$
\begin{gathered}
\tilde{U}\left(0, \eta, \frac{\Theta_{1}-\Omega_{1}}{\sigma_{1}^{2}}+\frac{2 \Omega_{1} z_{1}}{\sigma_{1}^{2}}, \frac{\Theta_{2}-\Omega_{2}}{\sigma_{2}^{2}}+\frac{\left(\sigma_{2}^{2} s_{2}-\Theta_{2}+\Omega_{2}\right) 2 \Omega_{2} z_{2}}{\left[\sigma_{2}^{2} s_{2}-\Theta_{2}+\Omega_{2}+2 \Omega_{2}\left(z_{2}-1\right)\right] \sigma_{2}^{2}}\right) \\
=\frac{\Gamma\left(\frac{2 \Phi_{1}}{\sigma_{1}^{2}}-1\right)}{\Gamma\left(\frac{2 \Phi_{1}}{\sigma_{1}^{2}}-1\right)} \int_{0}^{\infty} \int_{0}^{\infty} \hat{U}\left(0, \eta, v_{1}, v_{2}\right) \exp \left\{-\left[\frac{\Theta_{1}-\Omega_{1}}{\sigma_{1}^{2}}+\frac{2 \Omega_{1} z_{1}}{\sigma_{1}^{2}}\right] v_{1}\right\} \\
\quad \times \exp \left\{-\left[\frac{\Theta_{2}-\Omega_{2}}{\sigma_{2}^{2}}+\frac{\left(\sigma_{2}^{2} s_{2}-\Theta_{2}+\Omega_{2}\right) 2 \Omega_{2} z_{2}}{\left[\sigma_{2}^{2} s_{2}-\Theta_{2}+\Omega_{2}+2 \Omega_{2}\left(z_{2}-1\right)\right] \sigma_{2}^{2}}\right] v_{2}\right\} d v_{1} d v_{2} .
\end{gathered}
$$

Further manipulations yield

$$
\begin{aligned}
& \tilde{U}\left(0, \eta, \frac{\Theta_{1}-\Omega_{1}}{\sigma_{1}^{2}}+\frac{2 \Omega_{1} z_{1}}{\sigma_{1}^{2}}, \frac{\Theta_{2}-\Omega_{2}}{\sigma_{2}^{2}}+\frac{\left(\sigma_{2}^{2} s_{2}-\Theta_{2}+\Omega_{2}\right) 2 \Omega_{2} z_{2}}{\left[\sigma_{2}^{2} s_{2}-\Theta_{2}+\Omega_{2}+2 \Omega_{2}\left(z_{2}-1\right)\right] \sigma_{2}^{2}}\right) \\
& \quad=\frac{1}{\Gamma\left(\frac{2 \Phi_{1}}{\sigma_{1}^{2}}-1\right)} \int_{0}^{\infty} \int_{0}^{\infty} \int_{0}^{\infty} e^{-a_{1}} a_{1}^{\frac{2 \Phi_{1}}{\sigma_{1}^{2}}-2} \hat{U}\left(0, \eta, v_{1}, v_{2}\right) \exp \left\{-\left[\frac{\Theta_{1}-\Omega_{1}}{\sigma_{1}^{2}}+\frac{2 \Omega_{1} z_{1}}{\sigma_{1}^{2}}\right] v_{1}\right\} \\
& \quad \times \exp \left\{-\left[\frac{\Theta_{2}-\Omega_{2}}{\sigma_{2}^{2}}+\frac{\left(\sigma_{2}^{2} s_{2}-\Theta_{2}+\Omega_{2}\right) 2 \Omega_{2} z_{2}}{\left[\sigma_{2}^{2} s_{2}-\Theta_{2}+\Omega_{2}+2 \Omega_{2}\left(z_{2}-1\right)\right] \sigma_{2}^{2}}\right] v_{2}\right\} d a_{1} d v_{1} d v_{2} .
\end{aligned}
$$

Now, we make the substitution $a_{1}=\left(\frac{2 \Omega_{1} v_{1}}{\sigma_{1}^{2}}\right) y_{1}$ in equation (A4.35) and obtain

$$
\begin{aligned}
\tilde{U}(0, \eta, & \left.\frac{\Theta_{1}-\Omega_{1}}{\sigma_{1}^{2}}+\frac{2 \Omega_{1} z_{1}}{\sigma_{1}^{2}}, \frac{\Theta_{2}-\Omega_{2}}{\sigma_{2}^{2}}+\frac{\left(\sigma_{2}^{2} s_{2}-\Theta_{2}+\Omega_{2}\right) 2 \Omega_{2} z_{2}}{\left[\sigma_{2}^{2} s_{2}-\Theta_{2}+\Omega_{2}+2 \Omega_{2}\left(z_{2}-1\right)\right] \sigma_{2}^{2}}\right) \\
& =\frac{1}{\Gamma\left(\frac{2 \Phi_{1}}{\sigma_{1}^{2}}-1\right)} \int_{0}^{\infty} \int_{0}^{\infty} \int_{0}^{\infty} \exp \left\{-\left(\frac{2 \Omega_{1} v_{1}}{\sigma_{1}^{2}}\right) y_{1}\right\}\left(\frac{2 \Omega_{1} v_{1}}{\sigma_{1}^{2}} y_{1}\right)^{\frac{2 \Phi_{1}}{\sigma_{1}^{2}}-2} \hat{U}\left(0, \eta, v_{1}, v_{2}\right) \\
& \times \exp \left\{-\left(\frac{\Theta_{1}-\Omega_{1}}{\sigma_{1}^{2}}+\frac{2 \Omega_{1} z_{1}}{\sigma_{1}^{2}}\right) v_{1}\right\}\left(\frac{2 \Omega_{1} v_{1}}{\sigma_{1}^{2}}\right) \\
& \times \exp \left\{-\left(\frac{\Theta_{2}-\Omega_{2}}{\sigma_{2}^{2}}+\frac{\left(\sigma_{2}^{2} s_{2}-\Theta_{2}+\Omega_{2}\right) 2 \Omega_{2} z_{2}}{\left[\sigma_{2}^{2} s_{2}-\Theta_{2}+\Omega_{2}+2 \Omega_{2}\left(z_{2}-1\right)\right] \sigma_{2}^{2}}\right) v_{2}\right\} d y_{1} d v_{1} d v_{2} .
\end{aligned}
$$

\footnotetext{
${ }^{10}$ The choice of the gamma function $\Gamma\left(\frac{2 \Phi_{1}}{\sigma_{1}^{2}}-1\right)$ may seem arbitrary as it seems we could have chosen $\Gamma\left(\beta_{1}\right)$, for any $\beta_{1}$. However it turns out that to make equation (A4.34) match with (A4.32) we need to take $\beta_{1}=\frac{2 \Phi_{1}}{\sigma_{1}^{2}}-1$.
} 
Rearranging equation (A4.36) yields

$$
\begin{aligned}
\tilde{U}(0, \eta, & \left.\frac{\Theta_{1}-\Omega_{1}}{\sigma_{1}^{2}}+\frac{2 \Omega_{1} z_{1}}{\sigma_{1}^{2}}, \frac{\Theta_{2}-\Omega_{2}}{\sigma_{2}^{2}}+\frac{\left(\sigma_{2}^{2} s_{2}-\Theta_{2}+\Omega_{2}\right) 2 \Omega_{2} z_{2}}{\left[\sigma_{2}^{2} s_{2}-\Theta_{2}+\Omega_{2}+2 \Omega_{2}\left(z_{2}-1\right)\right] \sigma_{2}^{2}}\right) \\
& =\frac{1}{\Gamma\left(\frac{2 \Phi_{1}}{\sigma_{1}^{2}}-1\right)} \int_{0}^{\infty} \int_{0}^{\infty} \hat{U}\left(0, \eta, v_{1}, v_{2}\right) \exp \left\{-\left(\frac{\Theta_{1}-\Omega_{1}}{\sigma_{1}^{2}}\right) v_{1}\right\} \\
& \times \exp \left\{-\left(\frac{\Theta_{2}-\Omega_{2}}{\sigma_{2}^{2}}+\frac{\left(\sigma_{2}^{2} s_{2}-\Theta_{2}+\Omega_{2}\right) 2 \Omega_{2} z_{2}}{\left[\sigma_{2}^{2} s_{2}-\Theta_{2}+\Omega_{2}+2 \Omega_{2}\left(z_{2}-1\right)\right] \sigma_{2}^{2}}\right) v_{2}\right\} \\
& \times\left[\int_{0}^{\infty} \exp \left\{-\left(\frac{2 \Omega_{1} v_{1}}{\sigma_{1}^{2}}\right) y_{1}\right\}\left(\frac{2 \Omega_{1} v_{1}}{\sigma_{1}^{2}} y_{1}\right)^{\frac{2 \Phi_{1}}{\sigma_{1}^{2}}-2} \exp \left\{-\left(\frac{2 \Omega_{1} z_{1}}{\sigma_{1}^{2}}\right) v_{1}\right\} d y_{1}\right] d v_{1} d v_{2} .
\end{aligned}
$$

The terms inside the third integral component of equation (A4.37) can further be rearranged to yield

$$
\begin{aligned}
\tilde{U}(0, \eta, & \left.\frac{\Theta_{1}-\Omega_{1}}{\sigma_{1}^{2}}+\frac{2 \Omega_{1} z_{1}}{\sigma_{1}^{2}}, \frac{\Theta_{2}-\Omega_{2}}{\sigma_{2}^{2}}+\frac{\left(\sigma_{2}^{2} s_{2}-\Theta_{2}+\Omega_{2}\right) 2 \Omega_{2} z_{2}}{\left[\sigma_{2}^{2} s_{2}-\Theta_{2}+\Omega_{2}+2 \Omega_{2}\left(z_{2}-1\right)\right] \sigma_{2}^{2}}\right) \\
& =\frac{1}{\Gamma\left(\frac{2 \Phi_{1}}{\sigma_{1}^{2}}-1\right)} \int_{0}^{\infty} \int_{0}^{\infty} \hat{U}\left(0, \eta, v_{1}, v_{2}\right) \exp \left\{-\left(\frac{\Theta_{1}-\Omega_{1}}{\sigma_{1}^{2}}\right) v_{1}\right\} \\
& \times \exp \left\{-\left(\frac{\Theta_{2}-\Omega_{2}}{\sigma_{2}^{2}}+\frac{\left(\sigma_{2}^{2} s_{2}-\Theta_{2}+\Omega_{2}\right) 2 \Omega_{2} z_{2}}{\left[\sigma_{2}^{2} s_{2}-\Theta_{2}+\Omega_{2}+2 \Omega_{2}\left(z_{2}-1\right)\right] \sigma_{2}^{2}}\right) v_{2}\right\}\left(\frac{2 \Omega_{1} v_{1}}{\sigma_{1}^{2}}\right)^{\frac{2 \Phi_{1}}{\sigma_{1}^{2}}-1} \\
& \times\left[\int_{0}^{\infty} \exp \left\{-\left(\frac{2 \Omega_{1} v_{1}}{\sigma_{1}^{2}}\right)\left(y_{1}+z_{1}\right)\right\} y_{1}^{\frac{2 \Phi_{1}}{\sigma_{1}^{2}}-2} d y_{1}\right] d v_{1} d v_{2} .
\end{aligned}
$$

We make one further transformation by letting $\varrho_{1}=y_{1}+z_{1}$. Incorporating this in equation (A4.38) we obtain

$$
\begin{aligned}
\tilde{U}(0, \eta, & \left.\frac{\Theta_{1}-\Omega_{1}}{\sigma_{1}^{2}}+\frac{2 \Omega_{1} z_{1}}{\sigma_{1}^{2}}, \frac{\Theta_{2}-\Omega_{2}}{\sigma_{2}^{2}}+\frac{\left(\sigma_{2}^{2} s_{2}-\Theta_{2}+\Omega_{2}\right) 2 \Omega_{2} z_{2}}{\left[\sigma_{2}^{2} s_{2}-\Theta_{2}+\Omega_{2}+2 \Omega_{2}\left(z_{2}-1\right)\right] \sigma_{2}^{2}}\right) \\
& =\frac{1}{\Gamma\left(\frac{2 \Phi_{1}}{\sigma_{1}^{2}}-1\right)} \int_{0}^{\infty} \int_{0}^{\infty} \hat{U}\left(0, \eta, v_{1}, v_{2}\right) \exp \left\{-\left(\frac{\Theta_{1}-\Omega_{1}}{\sigma_{1}^{2}}\right) v_{1}\right\}\left(\frac{2 \Omega_{1} v_{1}}{\sigma_{1}^{2}}\right)^{\frac{2 \Phi_{1}}{\sigma_{1}^{2}}-1} \\
& \times \exp \left\{-\left(\frac{\Theta_{2}-\Omega_{2}}{\sigma_{2}^{2}}+\frac{\left(\sigma_{2}^{2} s_{2}-\Theta_{2}+\Omega_{2}\right) 2 \Omega_{2} z_{2}}{\left[\sigma_{2}^{2} s_{2}-\Theta_{2}+\Omega_{2}+2 \Omega_{2}\left(z_{2}-1\right)\right] \sigma_{2}^{2}}\right) v_{2}\right\} \\
& \times\left[\int_{z_{1}}^{\infty}\left(\varrho_{1}-z_{1}\right)^{\frac{2}{\sigma_{1}^{2}}\left(\Phi_{1}-\sigma_{1}^{2}\right)} \exp \left\{-\left(\frac{2 \Omega_{1} v_{1}}{\sigma_{1}^{2}}\right) \varrho_{1}\right\}\right] d \varrho_{1} d v_{1} d v_{2} .
\end{aligned}
$$


This last equation can be represented as

$$
\begin{aligned}
\tilde{U}\left(0, \eta, \frac{\Theta_{1}-\Omega_{1}}{\sigma_{1}^{2}}+\frac{2 \Omega_{1} z_{1}}{\sigma_{1}^{2}}, \frac{\Theta_{2}-\Omega_{2}}{\sigma_{2}^{2}}+\frac{\left(\sigma_{2}^{2} s_{2}-\Theta_{2}+\Omega_{2}\right) 2 \Omega_{2} z_{2}}{\left[\sigma_{2}^{2} s_{2}-\Theta_{2}+\Omega_{2}+2 \Omega_{2}\left(z_{2}-1\right)\right] \sigma_{2}^{2}}\right) \\
=\int_{z_{1}}^{\infty}\left(\varrho_{1}-z_{1}\right)^{\frac{2}{\sigma_{1}^{2}}\left(\Phi_{1}-\sigma_{1}^{2}\right)}\left[\int_{0}^{\infty} \int_{0}^{\infty} \frac{\hat{U}\left(0, \eta, v_{1}, v_{2}\right)}{\Gamma\left(\frac{2 \Phi_{1}}{\sigma_{1}^{2}}-1\right)}\left(\frac{2 \Omega_{1} v_{1}}{\sigma_{1}^{2}}\right)^{\frac{2 \Phi_{1}}{\sigma_{1}^{2}}-1} \exp \left\{-\left(\frac{\Theta_{1}-\Omega_{1}}{\sigma_{1}^{2}}+\frac{2 \Omega_{1} \varrho_{1}}{\sigma_{1}^{2}}\right) v_{1}\right\}\right. \\
\left.\quad \times \exp \left\{-\left(\frac{\Theta_{2}-\Omega_{2}}{\sigma_{2}^{2}}+\frac{\left(\sigma_{2}^{2} s_{2}-\Theta_{2}+\Omega_{2}\right) 2 \Omega_{2} z_{2}}{\left[\sigma_{2}^{2} s_{2}-\Theta_{2}+\Omega_{2}+2 \Omega_{2}\left(z_{2}-1\right)\right] \sigma_{2}^{2}}\right) v_{2}\right\} d v_{1} d v_{2}\right] d \varrho_{1} . \quad(\mathrm{A} 4.40)
\end{aligned}
$$

By comparing equations (A4.32) and (A4.40) we have in fact shown that

$$
\begin{aligned}
g_{1}\left(\zeta_{1}\right) & =-\Omega_{1} \int_{0}^{\infty} \int_{0}^{\infty} \frac{\hat{U}\left(0, \eta, v_{1}, v_{2}\right)}{\Gamma\left(\frac{2 \Phi_{1}}{\sigma_{1}^{2}}-1\right)}\left(\frac{2 \Omega_{1} v_{1}}{\sigma_{1}^{2}}\right)^{\frac{2 \Phi_{1}}{\sigma_{1}^{2}}-1} \exp \left\{-\left(\frac{\Theta_{1}-\Omega_{1}}{\sigma_{1}^{2}}+\frac{2 \Omega_{1} \zeta_{1}}{\sigma_{1}^{2}}\right) v_{1}\right\} \\
& \times \exp \left\{-\left(\frac{\Theta_{2}-\Omega_{2}}{\sigma_{2}^{2}}+\frac{\left(\sigma_{2}^{2} s_{2}-\Theta_{2}+\Omega_{2}\right) 2 \Omega_{2} z_{2}}{\left[\sigma_{2}^{2} s_{2}-\Theta_{2}+\Omega_{2}+2 \Omega_{2}\left(z_{2}-1\right)\right] \sigma_{2}^{2}}\right) v_{2}\right\} d v_{1} d v_{2}
\end{aligned}
$$

We recall from equation (5.3) that the initial condition is expressed as

$$
\hat{U}\left(0, \eta, v_{1}, v_{2}\right)=e^{i \eta x_{0}} \delta\left(v_{1}-v_{1,0}\right) \delta\left(v_{2}-v_{2,0}\right) .
$$

Substituting this into equation (A4.41) we obtain

$$
\begin{aligned}
g_{1}\left(\zeta_{1}\right) & =-\Omega_{1} \int_{0}^{\infty} \int_{0}^{\infty} \frac{\delta\left(v_{1}-v_{1,0}\right) \delta\left(v_{2}-v_{2,0}\right)}{\Gamma\left(\frac{2 \Phi_{1}}{\sigma_{1}^{2}}-1\right)}\left(\frac{2 \Omega_{1} v_{1}}{\sigma_{1}^{2}}\right)^{\frac{2 \Phi_{1}}{\sigma_{1}^{2}}-1} \exp \left\{-\left(\frac{\Theta_{1}-\Omega_{1}}{\sigma_{1}^{2}}+\frac{2 \Omega_{1} \zeta_{1}}{\sigma_{1}^{2}}\right) v_{1}+i \eta x_{0}\right\} \\
& \times \exp \left\{-\left(\frac{\Theta_{2}-\Omega_{2}}{\sigma_{2}^{2}}+\frac{\left(\sigma_{2}^{2} s_{2}-\Theta_{2}+\Omega_{2}\right) 2 \Omega_{2} z_{2}}{\left[\sigma_{2}^{2} s_{2}-\Theta_{2}+\Omega_{2}+2 \Omega_{2}\left(z_{2}-1\right)\right] \sigma_{2}^{2}}\right) v_{2}\right\} d v_{1} d v_{2} .
\end{aligned}
$$

Using properties of the delta functions the above expression simplifies to

$$
\begin{aligned}
g_{1}\left(\zeta_{1}\right) & =\frac{-\Omega_{1}}{\Gamma\left(\frac{2 \Phi_{1}}{\sigma_{1}^{2}}-1\right)}\left(\frac{2 \Omega_{1} v_{1,0}}{\sigma_{1}^{2}}\right)^{\frac{2 \Phi_{1}}{\sigma_{1}^{2}}-1} \exp \left\{-\left(\frac{\Theta_{1}-\Omega_{1}}{\sigma_{1}^{2}}+\frac{2 \Omega_{1} \zeta_{1}}{\sigma_{1}^{2}}\right) v_{1,0}+i \eta x_{0}\right\} \\
& \times \exp \left\{-\left(\frac{\Theta_{2}-\Omega_{2}}{\sigma_{2}^{2}}+\frac{\left(\sigma_{2}^{2} s_{2}-\Theta_{2}+\Omega_{2}\right) 2 \Omega_{2} z_{2}}{\left[\sigma_{2}^{2} s_{2}-\Theta_{2}+\Omega_{2}+2 \Omega_{2}\left(z_{2}-1\right)\right] \sigma_{2}^{2}}\right) v_{2,0}\right\} .
\end{aligned}
$$

Given the explicit representation of $g_{1}\left(\zeta_{1}\right)$ we can now find the explicit form of the 
function $f_{1}\left(t, s_{2}\right)$ by comparing equations (A4.31) and (A4.44) such that ${ }^{11}$

$$
\begin{aligned}
f_{1}\left(t, s_{2}\right) & =\frac{-\Omega_{1}}{\Gamma\left(\frac{2 \Phi_{1}}{\sigma_{1}^{2}}-1\right)}\left(\frac{2 \Omega_{1} v_{1,0}}{\sigma_{1}^{2}}\right)^{\frac{2 \Phi_{1}}{\sigma_{1}^{2}}-1} \exp \left\{-\left(\frac{\Theta_{1}-\Omega_{1}}{\sigma_{1}^{2}}+\frac{2 \Omega_{1} \zeta_{1}}{\sigma_{1}^{2}}\right) v_{1,0}+i \eta x_{0}\right\} \\
& \times \exp \left\{-\left(\frac{\Theta_{2}-\Omega_{2}}{\sigma_{2}^{2}}+\frac{\left(\sigma_{2}^{2} s_{2}-\Theta_{2}+\Omega_{2}\right) 2 \Omega_{2} z_{2}}{\left[\sigma_{2}^{2} s_{2}-\Theta_{2}+\Omega_{2}+2 \Omega_{2}\left(z_{2}-1\right)\right] \sigma_{2}^{2}}\right) v_{2,0}\right\} \\
& \times \exp \left\{\left[\frac{\left(\Phi_{1}-\sigma_{1}^{2}\right)\left(\Theta_{1}-\Omega_{1}\right)}{\sigma_{1}^{2}}+\frac{\left(\Phi_{2}-\sigma_{2}^{2}\right)\left(\Theta_{2}-\Omega_{2}\right)}{\sigma_{2}^{2}}-i \eta(r-q)+\Theta_{1}+\Theta_{2}\right] t\right\} \\
& \times\left(\frac{\left(\sigma_{2}^{2} s_{2}-\Theta_{2}+\Omega_{2}\right)\left(\zeta_{2}-z_{2}\right)+2 \Omega_{2} \zeta_{2}\left(z_{2}-1\right)}{\zeta_{2}\left[\left(\sigma_{2}^{2} s_{2}-\Theta_{2}+\Omega_{2}\right)+2 \Omega_{2}\left(z_{2}-1\right)\right]}\right)^{\frac{2}{\sigma_{2}^{2}}\left(\sigma_{2}^{2}-\Phi_{2}\right)} \frac{\zeta_{1}\left(\zeta_{1}-1\right)}{\zeta_{1}^{\frac{2}{\sigma_{1}^{2}\left(\sigma_{1}^{2}-\Phi_{1}\right)}} .}
\end{aligned}
$$

By performing similar operations it can be shown that

$$
\begin{aligned}
f_{2}\left(t, s_{1}\right) & =\frac{-\Omega_{2}}{\Gamma\left(\frac{2 \Phi_{2}}{\sigma_{2}^{2}}-1\right)}\left(\frac{2 \Omega_{2} v_{2,0}}{\sigma_{2}^{2}}\right)^{\frac{2 \Phi_{2}}{\sigma_{2}^{2}}-1} \exp \left\{-\left(\frac{\Theta_{1}-\Omega_{2}}{\sigma_{2}^{2}}+\frac{2 \Omega_{2} \zeta_{2}}{\sigma_{2}^{2}}\right) v_{2,0}+i \eta x_{0}\right\} \\
& \times \exp \left\{-\left(\frac{\Theta_{1}-\Omega_{1}}{\sigma_{1}^{2}}+\frac{\left(\sigma_{1}^{2} s_{1}-\Theta_{1}+\Omega_{1}\right) 2 \Omega_{1} z_{1}}{\left[\sigma_{1}^{2} s_{1}-\Theta_{1}+\Omega_{1}+2 \Omega_{1}\left(z_{1}-1\right)\right] \sigma_{1}^{2}}\right) v_{1,0}\right\} \\
& \times \exp \left\{\left[\frac{\left(\Phi_{1}-\sigma_{1}^{2}\right)\left(\Theta_{1}-\Omega_{1}\right)}{\sigma_{1}^{2}}+\frac{\left(\Phi_{2}-\sigma_{2}^{2}\right)\left(\Theta_{2}-\Omega_{2}\right)}{\sigma_{2}^{2}}-i \eta(r-q)+\Theta_{1}+\Theta_{2}\right] t\right\} \\
& \times\left(\frac{\left(\sigma_{1}^{2} s_{1}-\Theta_{1}+\Omega_{1}\right)\left(\zeta_{1}-z_{1}\right)+2 \Omega_{1} \zeta_{1}\left(z_{1}-1\right)}{\zeta_{1}\left[\left(\sigma_{1}^{2} s_{1}-\Theta_{1}+\Omega_{1}\right)+2 \Omega_{1}\left(z_{1}-1\right)\right]}\right)^{\frac{2}{\sigma_{1}^{2}}\left(\sigma_{1}^{2}-\Phi_{1}\right)} \frac{\zeta_{2}\left(\zeta_{2}-1\right)}{\zeta_{2}^{\frac{2}{\sigma_{2}^{2}}\left(\sigma_{2}^{2}-\Phi_{2}\right)}} .
\end{aligned}
$$

\section{Deriving the explicit representation of $\tilde{\mathbf{U}}\left(\tau, \eta, \mathbf{s}_{\mathbf{1}}, \mathbf{s}_{\mathbf{2}}\right)$ :}

Now that we have found the two unknown functions namely $f_{1}\left(t, s_{2}\right)$ and $f_{2}\left(t, s_{1}\right)$, the next step is to substitute these two functions into equation (A4.27) in order for us to finally obtain the representation of the transform. We are going to do this in three steps. We break equation (A4.27) into three parts. The first part being the first term on the RHS of (A4.27), the second part is the term involving $f_{1}\left(t, s_{2}\right)$ and the third part is the one involving the $f_{2}\left(t, s_{1}\right)$ term.

\footnotetext{
${ }^{11}$ In actual fact, from equations (A4.7) and (A4.12) $s_{j}=s_{j}(\tau)$ and from equation (A4.29) $\zeta_{j}=\zeta_{j}(t)$ for $j=1,2$. We suppress the dependence on time for convenience.
} 
The first component on the RHS of (A4.27) can be represented as

$$
\begin{aligned}
& J_{1}= \exp \left\{\left[\frac{\left(\Phi_{1}-\sigma_{1}^{2}\right)\left(\Theta_{1}-\Omega_{1}\right)}{\sigma_{1}^{2}}+\frac{\left(\Phi_{2}-\sigma_{2}^{2}\right)\left(\Theta_{2}-\Omega_{2}\right)}{\sigma_{2}^{2}}-i \eta(r-q)+\Theta_{1}+\Theta_{2}\right] \tau\right\} \\
& \times\left.\left(\frac{2 \Omega_{1} e^{-\Omega_{1} \tau}}{\left(\sigma_{1}^{2} s_{1}-\Theta_{1}+\Omega_{1}\right)\left(1-e^{-\Omega_{1} \tau}\right)+2 \Omega_{1} e^{-\Omega_{1} \tau}}\right)^{\frac{2}{\sigma_{1}^{2}\left(\sigma_{1}^{2}-\Phi_{1}\right)}}\right)^{\frac{2}{\sigma_{2}^{2}}\left(\sigma_{2}^{2}-\Phi_{2}\right)} \\
& \times\left(\frac{2 \Omega_{2} e^{-\Omega_{2} \tau}}{\left(\sigma_{2}^{2} s_{2}-\Theta_{2}+\Omega_{2}\right)\left(1-e^{-\Omega_{2} \tau}\right)+2 \Omega_{2} e^{-\Omega_{2} \tau}}\right)^{2 \Omega_{1}\left(\sigma_{1}^{2} s_{1}-\Theta_{1}+\Omega_{1}\right)} \\
& \times \tilde{U}\left(0, \eta, \frac{\Theta_{1}-\Omega_{1}}{\sigma_{1}^{2}}-\frac{2 \Omega_{2}\left(\sigma_{2}^{2} s_{2}-\Theta_{2}+\Omega_{2}\right)}{\sigma_{1}^{2}\left[\left(\sigma_{1}^{2} s_{1}-\Theta_{1}+\Omega_{1}\right)\left(e^{-\Omega_{1} \tau}-1\right)-2 \Omega_{1} e^{-\Omega_{1} \tau}\right]},\right. \\
&\left.\frac{\Theta_{2}-\Omega_{2}}{\sigma_{2}^{2}}-\frac{\Theta_{2}}{\sigma_{2}^{2}\left[\left(\sigma_{2}^{2} s_{2}-\Theta_{2}+\Omega_{2}\right)\left(e^{-\Omega_{2} \tau}-1\right)-2 \Omega_{2} e^{-\Omega_{2} \tau}\right]}\right) .
\end{aligned}
$$

Making use of equation (A4.30) we obtain

$$
\begin{aligned}
& J_{1}=\exp \left\{\left[\frac{\left(\Phi_{1}-\sigma_{1}^{2}\right)\left(\Theta_{1}-\Omega_{1}\right)}{\sigma_{1}^{2}}+\frac{\left(\Phi_{2}-\sigma_{2}^{2}\right)\left(\Theta_{2}-\Omega_{2}\right)}{\sigma_{2}^{2}}-i \eta(r-q)+\Theta_{1}+\Theta_{2}\right] \tau\right\} \\
& \times\left(\frac{2 \Omega_{1}\left(z_{1}-1\right)}{\left(\sigma_{1}^{2} s_{1}-\Theta_{1}+\Omega_{1}\right)+2 \Omega_{1}\left(z_{1}-1\right)}\right)^{2-\frac{2 \Phi_{1}}{\sigma_{1}^{2}}}\left(\frac{2 \Omega_{2}\left(z_{2}-1\right)}{\left(\sigma_{2}^{2} s_{2}-\Theta_{2}+\Omega_{2}\right)+2 \Omega_{2}\left(z_{2}-1\right)}\right)^{2-\frac{2 \Phi_{2}}{\sigma_{2}^{2}}} \\
& \times \tilde{U}\left(0, \eta, \frac{\Theta_{1}-\Omega_{1}}{\sigma_{1}^{2}}+\frac{2 \Omega_{1}\left(\sigma_{1}^{2} s_{1}-\Theta_{1}+\Omega_{1}\right) z_{1}}{\sigma_{1}^{2}\left[\left(\sigma_{1}^{2} s_{1}-\Theta_{1}+\Omega_{1}\right)+2 \Omega_{1}\left(z_{1}-1\right)\right]}, \frac{\Theta_{2}-\Omega_{2}}{\sigma_{2}^{2}}+\frac{2 \Omega_{2}\left(\sigma_{2}^{2} s_{2}-\Theta_{2}+\Omega_{2}\right) z_{2}}{\sigma_{2}^{2}\left[\left(\sigma_{2}^{2} s_{2}-\Theta_{2}+\Omega_{2}\right)+2 \Omega_{2}\left(z_{2}-1\right)\right]}\right) .
\end{aligned}
$$

Applying the initial condition (6.2) to equation (A4.48) yields

$$
\begin{aligned}
J_{1} & =\left(\frac{2 \Omega_{1}\left(z_{1}-1\right)}{\left(\sigma_{1}^{2} s_{1}-\Theta_{1}+\Omega_{1}\right)+2 \Omega_{1}\left(z_{1}-1\right)}\right)^{2-\frac{2 \Phi_{1}}{\sigma_{1}^{2}}}\left(\frac{2 \Omega_{2}\left(z_{2}-1\right)}{\left(\sigma_{2}^{2} s_{2}-\Theta_{2}+\Omega_{2}\right)+2 \Omega_{2}\left(z_{2}-1\right)}\right)^{2-\frac{2 \Phi_{2}}{\sigma_{2}^{2}}} \\
& \times \exp \left\{\left[\frac{\left(\Phi_{1}-\sigma_{1}^{2}\right)\left(\Theta_{1}-\Omega_{1}\right)}{\sigma_{1}^{2}}+\frac{\left(\Phi_{2}-\sigma_{2}^{2}\right)\left(\Theta_{2}-\Omega_{2}\right)}{\sigma_{2}^{2}}-i \eta(r-q)+\Theta_{1}+\Theta_{2}\right] \tau\right\} \\
& \times \exp \left\{-\left(\frac{\Theta_{1}-\Omega_{1}}{\sigma_{1}^{2}}\right) v_{1,0}-\left(\frac{\Theta_{2}-\Omega_{2}}{\sigma_{2}^{2}}\right) v_{2,0}+i \eta x_{0}\right\} \\
& \times \exp \left\{\frac{-2 \Omega_{1} v_{1,0}\left(\sigma_{1}^{2} s_{1}-\Theta_{1}+\Omega_{1}\right) z_{1}}{\sigma_{1}^{2}\left[\left(\sigma_{1}^{2} s_{1}-\Theta_{1}+\Omega_{1}\right)+2 \Omega_{1}\left(z_{1}-1\right)\right]}\right\} \exp \left\{\frac{-2 \Omega_{2} v_{2,0}\left(\sigma_{2}^{2} s_{2}-\Theta_{2}+\Omega_{2}\right) z_{2}}{\sigma_{2}^{2}\left[\left(\sigma_{2}^{2} s_{2}-\Theta_{2}+\Omega_{2}\right)+2 \Omega_{2}\left(z_{2}-1\right)\right]}\right\}
\end{aligned}
$$

The second component is here represented as $^{12}$

\footnotetext{
${ }^{12}$ We recall the link between $\zeta_{1}$ and $t$ from (A4.29) and that between $z_{1}$ and $\tau$ from (A4.30).
} 


$$
\begin{gathered}
J_{2}=\frac{1}{\Omega_{1}} \int_{z_{1}}^{\infty} f_{1}\left(t, s_{2}\right) \exp \left\{\left[\frac{\left(\Phi_{1}-\sigma_{1}^{2}\right)\left(\Theta_{1}-\Omega_{1}\right)}{\sigma_{1}^{2}}+\frac{\left(\Phi_{2}-\sigma_{2}^{2}\right)\left(\Theta_{2}-\Omega_{2}\right)}{\sigma_{2}^{2}}-i \eta(r-q)+\Theta_{1}+\Theta_{2}\right](\tau-t)\right\} \\
\quad \times\left(\frac{2 \Omega_{1} \zeta_{1}\left(z_{1}-1\right)}{\left(\sigma_{1}^{2} s_{1}-\Theta_{1}+\Omega_{1}\right)\left(\zeta_{1}-z_{1}\right)+2 \Omega_{1} \zeta_{1}\left(z_{1}-1\right)}\right)^{\frac{2}{\sigma_{1}^{2}\left(\sigma_{1}^{2}-\Phi_{1}\right)}} \\
\quad \times\left(\frac{2 \Omega_{2} \zeta_{2}\left(z_{2}-1\right)}{\left(\sigma_{2}^{2} s_{2}-\Theta_{2}+\Omega_{2}\right)\left(\zeta_{2}-z_{2}\right)+2 \Omega_{2} \zeta_{2}\left(z_{2}-1\right)}\right)^{\frac{2}{\sigma_{2}^{2}}\left(\sigma_{2}^{2}-\Phi_{2}\right)} \frac{d \zeta_{1}}{\zeta_{1}\left(\zeta_{1}-1\right)} .
\end{gathered}
$$

Now by substituting the value of $f_{1}\left(t, s_{2}\right)$ in equation (A4.45) into equation (A4.50) we obtain

$$
\begin{aligned}
J_{2}= & \frac{-1}{\Gamma\left(\frac{2 \Phi_{1}}{\sigma_{1}^{2}}-1\right)} \int_{z_{1}}^{\infty}\left(\frac{2 \Omega_{1} v_{1,0}}{\sigma_{1}^{2}}\right)^{\frac{2 \Phi_{1}}{\sigma_{1}^{2}}-1} \exp \left\{-\left(\frac{\Theta_{1}-\Omega_{1}}{\sigma_{1}^{2}}+\frac{2 \Omega_{1} \zeta_{1}}{\sigma_{1}^{2}}\right) v_{1,0}+i \eta x_{0}\right\} \\
\times \exp & \left\{-\left(\frac{\Theta_{2}-\Omega_{2}}{\sigma_{2}^{2}}+\frac{\left(\sigma_{2}^{2} s_{2}-\Theta_{2}+\Omega_{2}\right) 2 \Omega_{2} z_{2}}{\left[\sigma_{2}^{2} s_{2}-\Theta_{2}+\Omega_{2}+2 \Omega_{2}\left(z_{2}-1\right)\right] \sigma_{2}^{2}}\right) v_{2,0}\right\} \\
\times \exp \{ & \left.\left\{\frac{\left(\Phi_{1}-\sigma_{1}^{2}\right)\left(\Theta_{1}-\Omega_{1}\right)}{\sigma_{1}^{2}}+\frac{\left(\Phi_{2}-\sigma_{2}^{2}\right)\left(\Theta_{2}-\Omega_{2}\right)}{\sigma_{2}^{2}}-i \eta(r-q)+\Theta_{1}+\Theta_{2}\right] \tau\right\} \\
\times & \left.\left(\frac{2 \Omega_{1}\left(z_{1}-1\right)}{\left(\sigma_{1}^{2} s_{1}-\Theta_{1}+\Omega_{1}\right)\left(\zeta_{1}-z_{1}\right)+2 \Omega_{1} \zeta_{1}\left(z_{1}-1\right)}\right)^{\frac{2}{\sigma_{1}^{2}}\left(\sigma_{1}^{2}-\Phi_{1}\right)}\right)^{\frac{2}{\sigma_{2}^{2}}\left(\sigma_{2}^{2}-\Phi_{2}\right)} d \zeta_{1} . \\
& \times\left(\frac{2 \Omega_{2}\left(z_{2}-1\right)}{\sigma_{2}^{2} s_{2}-\Theta_{2}+\Omega_{2}+2 \Omega_{2}\left(z_{2}-1\right)}\right)^{(\mathrm{A} 4)}
\end{aligned}
$$

By rearranging the respective components of equation (A4.51) we obtain

$$
\begin{aligned}
J_{2}= & \frac{-\left[2 \Omega_{1}\left(z_{1}-1\right)\right]^{2-\frac{2 \Phi_{1}}{\sigma_{1}^{2}}}}{\Gamma\left(\frac{2 \Phi_{1}}{\sigma_{1}^{2}}-1\right)}\left(\frac{2 \Omega_{1} v_{1,0}}{\sigma_{1}^{2}}\right)^{\frac{2 \Phi_{1}}{\sigma_{1}^{2}}-1} \exp \left\{-\left(\frac{\Theta_{1}-\Omega_{1}}{\sigma_{1}^{2}}\right) v_{1,0}+i \eta x_{0}\right\} \\
\times & \exp \left\{-\left(\frac{\Theta_{2}-\Omega_{2}}{\sigma_{2}^{2}}+\frac{\left(\sigma_{2}^{2} s_{2}-\Theta_{2}+\Omega_{2}\right) 2 \Omega_{2} z_{2}}{\left[\sigma_{2}^{2} s_{2}-\Theta_{2}+\Omega_{2}+2 \Omega_{2}\left(z_{2}-1\right)\right] \sigma_{2}^{2}}\right) v_{2,0}\right\} \\
& \times \exp \left\{\left[\frac{\left(\Phi_{1}-\sigma_{1}^{2}\right)\left(\Theta_{1}-\Omega_{1}\right)}{\sigma_{1}^{2}}+\frac{\left(\Phi_{2}-\sigma_{2}^{2}\right)\left(\Theta_{2}-\Omega_{2}\right)}{\sigma_{2}^{2}}-i \eta(r-q)+\Theta_{1}+\Theta_{2}\right] \tau\right\} \\
& \times\left(\frac{2 \Omega_{2}\left(z_{2}-1\right)}{\sigma_{2}^{2} s_{2}-\Theta_{2}+\Omega_{2}+2 \Omega_{2}\left(z_{2}-1\right)}\right)^{\frac{2}{\sigma_{2}^{2}}\left(\sigma_{2}^{2}-\Phi_{2}\right)} G_{1}\left(v_{1,0}\right),
\end{aligned}
$$

where

$$
G_{1}\left(v_{1}\right)=\int_{z_{1}}^{\infty} e^{-\frac{2 \Omega_{1} v_{1}}{\sigma_{1}^{2}} \zeta_{1}}\left[\left(\sigma_{1}^{2} s_{1}-\Theta_{1}+\Omega_{1}\right)\left(\zeta_{1}-z_{1}\right)+2 \Omega_{1} \zeta_{1}\left(z_{1}-1\right)\right]^{\frac{2 \Phi_{1}}{\sigma_{1}^{2}}-2} d \zeta_{1} .
$$

As a way of simplifying equation (A4.53), we let $y_{1}=\left(\sigma_{1}^{2} s_{1}-\Theta_{1}+\Omega_{1}\right)\left(\zeta_{1}-z_{1}\right)+$ 
$2 \Omega_{1} \zeta_{1}\left(z_{1}-1\right)$ so that

$$
d \zeta_{1}=\frac{d y_{1}}{\sigma_{1}^{2} s_{1}-\Theta_{1}+\Omega_{1}+2 \Omega_{1}\left(z_{1}-1\right)}
$$

Substituting this into equation (A4.53) and rearranging terms we obtain

$$
\begin{aligned}
G_{1}\left(v_{1}\right) & =\frac{1}{\sigma_{1}^{2}-\Theta_{1}+\Omega_{1}+2 \Omega_{1}\left(z_{1}-1\right)} \exp \left\{-\frac{2 \Omega_{1} v_{1}}{\sigma_{1}^{2}}\left(\frac{\left(\sigma_{1}^{2} s_{1}-\Theta_{1}+\Omega_{1}\right) z_{1}}{\sigma_{1}^{2} s_{1}-\Theta_{1}+\Omega_{1}+2 \Omega_{1}\left(z_{1}-1\right)}\right)\right\} \\
& \times \int_{2 \Omega_{1} z_{1}\left(z_{1}-1\right)}^{\infty} \exp \left\{\frac{-2 \Omega_{1} v_{1} y_{1}}{\sigma_{1}^{2}\left[\sigma_{1}^{2} s_{1}-\Theta_{1}+\Omega_{1}+2 \Omega_{1}\left(z_{1}-1\right)\right]}\right\} y_{1}^{\frac{2 \Phi_{1}}{\sigma_{1}^{2}}-2} d y_{1} .
\end{aligned}
$$

Now let

$$
\xi_{1}=\frac{2 \Omega_{1} v_{1} y_{1}}{\sigma_{1}^{2}\left[\sigma_{1}^{2} s_{1}-\Theta_{1}+\Omega_{1}+2 \Omega_{1}\left(z_{1}-1\right)\right]}
$$

which implies that

$$
d y_{1}=\frac{\sigma_{1}^{2}\left[\sigma_{1}^{2} s_{1}-\Theta_{1}+\Omega_{1}+2 \Omega_{1}\left(z_{1}-1\right)\right]}{2 \Omega_{1} v_{1}} d \xi_{1} .
$$

Substituting these into equation (A4.54) yields

$$
\begin{aligned}
G_{1}\left(v_{1}\right) & =\frac{1}{\sigma_{1}^{2} s_{1}-\Theta_{1}+\Omega_{1}+2 \Omega_{1}\left(z_{1}-1\right)} \exp \left\{-\frac{2 \Omega_{1} v_{1}}{\sigma_{1}^{2}}\left(\frac{\left(\sigma_{1}^{2} s_{1}-\Theta_{1}+\Omega_{1}\right) z_{1}}{\sigma_{1}^{2} s_{1}-\Theta_{1}+\Omega_{1}+2 \Omega_{1}\left(z_{1}-1\right)}\right)\right\} \\
& \times \int_{\frac{4 \Omega_{1}^{2} v_{1} z_{1}\left(z_{1}-1\right)}{\sigma_{1}^{2}\left[\sigma_{1}^{2} s_{1}-\Theta_{1}+\Omega_{1}+2 \Omega_{1}\left(z_{1}-1\right)\right]}}^{\infty} e^{-\xi_{1}\left(\frac{\sigma_{1}^{2}\left[\sigma_{1}^{2} s_{1}-\Theta_{1}+\Omega_{1}+2 \Omega_{1}\left(z_{1}-1\right)\right] \xi_{1}}{2 \Omega_{1} v_{1}}\right)^{\frac{2 \Phi_{1}}{\sigma_{1}^{2}}-2}} \\
& \times \frac{\sigma_{1}^{2}\left[\sigma_{1}^{2} s_{1}-\Theta_{1}+\Omega_{1}+2 \Omega_{1}\left(z_{1}-1\right)\right]}{2 \Omega_{1} v_{1}} d \xi_{1} \\
& =\frac{\sigma_{1}^{2}}{2 \Omega_{1} v_{1}} \exp \left\{-\frac{2 \Omega_{1} v_{1}}{\sigma_{1}^{2}}\left(\frac{\left(\sigma_{1}^{2} s_{1}-\Theta_{1}+\Omega_{1}\right) z_{1}}{\sigma_{1}^{2} s_{1}-\Theta_{1}+\Omega_{1}+2 \Omega_{1}\left(z_{1}-1\right)}\right)\right\} \\
& \times\left(\frac{\sigma_{1}^{2}\left[\sigma_{1}^{2} s_{1}-\Theta_{1}+\Omega_{1}+2 \Omega_{1}\left(z_{1}-1\right)\right]}{2 \Omega_{1} v_{1}}\right)^{\frac{2 \Phi_{1}}{\sigma_{1}^{2}-2}} \int_{\frac{4 \Omega_{1}^{2} v_{1} z_{1}\left(z_{1}-1\right)}{\sigma_{1}^{2}\left[\sigma_{1}^{2} s_{1}-\Theta_{1}+\Omega_{1}+2 \Omega_{1}\left(z_{1}-1\right)\right]}}^{\infty} e^{-\xi_{1}} \xi_{1}^{\left(\frac{2 \Omega_{1}}{\left.\sigma_{1}^{2}-1\right)-1} d \xi_{1} .\right.}
\end{aligned}
$$

Rearranging and recalling the definition of the gamma function (see equation (7.4)) equation (A4.55) can be expressed as

$$
\begin{aligned}
G_{1}\left(v_{1}\right) & =\left[\sigma_{1}^{2} s_{1}-\Theta_{1}+\Omega_{1}+2 \Omega_{1}\left(z_{1}-1\right)\right]^{\frac{2 \Phi_{1}}{\sigma_{1}^{2}}-2}\left(\frac{\sigma_{1}^{2}}{2 \Omega_{1} v_{1}}\right)^{\frac{2 \Phi_{1}}{\sigma_{1}^{2}}-1} \exp \left\{\frac{-2 \Omega_{1} v_{1}\left(\sigma_{1}^{2} s_{1}-\Theta_{1}+\Omega_{1}\right) z_{1}}{\sigma_{1}^{2}\left[\sigma_{1}^{2} s_{1}-\Theta_{1}+\Omega_{1}+2 \Omega_{1}\left(z_{1}-1\right)\right]}\right\} \\
& \times\left[\Gamma\left(\frac{2 \Phi_{1}}{\sigma_{1}^{2}}-1\right)-\int_{0}^{\frac{4 \Omega_{1}^{2} v_{1} z_{1}\left(z_{1}-1\right)}{\sigma_{1}^{2}\left[\sigma_{1}^{2} s_{1}-\Theta_{1}+\Omega_{1}+2 \Omega_{1}\left(z_{1}-1\right)\right]}} e^{-\xi_{1}} \xi_{1}^{\left(\frac{2 \Omega_{1}}{\sigma_{1}^{2}}-1\right)-1} d \xi_{1}\right] .
\end{aligned}
$$

Substituting equation (A4.56) into (A4.52) and making use of equation (7.3) for the 
incomplete gamma function we obtain

$$
\begin{aligned}
J_{2}= & \frac{-1}{\Gamma\left(\frac{2 \Phi_{1}}{\sigma_{1}^{2}}-1\right)}\left(\frac{2 \Omega_{1}\left(z_{1}-1\right)}{\sigma_{1}^{2} s_{1}-\Theta_{1}+\Omega_{1}+2 \Omega_{1}\left(z_{1}-1\right)}\right)^{2-\frac{2 \Phi_{1}}{\sigma_{1}^{2}}}\left(\frac{2 \Omega_{2}\left(z_{2}-1\right)}{\sigma_{2}^{2} s_{2}-\Theta_{2}+\Omega_{2}+2 \Omega_{2}\left(z_{2}-1\right)}\right)^{2-\frac{2 \Phi_{2}}{\sigma_{2}^{2}}} \\
\times & \exp \left\{-\left(\frac{\Theta_{1}-\Omega_{1}}{\sigma_{1}^{2}}\right) v_{1,0}-\left(\frac{\Theta_{2}-\Omega_{2}}{\sigma_{2}^{2}}\right) v_{2,0}+i \eta x_{0}\right\} \\
& \times \exp \left\{\frac{-2 \Omega_{1} v_{1,0}\left(\sigma_{1}^{2} s_{1}-\Theta_{1}+\Omega_{1}\right) z_{1}}{\sigma_{1}^{2} s_{1}-\Theta_{1}+\Omega_{1}+2 \Omega_{1}\left(z_{1}-1\right)}\right\} \exp \left\{\frac{-2 \Omega_{2} v_{2,0}\left(\sigma_{2}^{2} s_{2}-\Theta_{2}+\Omega_{2}\right) z_{2}}{\left[\sigma_{2}^{2} s_{2}-\Theta_{2}+\Omega_{2}+2 \Omega_{2}\left(z_{2}-1\right)\right] \sigma_{2}^{2}}\right\} \\
& \times \exp \left\{\left[\frac{\left(\Phi_{1}-\sigma_{1}^{2}\right)\left(\Theta_{1}-\Omega_{1}\right)}{\sigma_{1}^{2}}+\frac{\left(\Phi_{2}-\sigma_{2}^{2}\right)\left(\Theta_{2}-\Omega_{2}\right)}{\sigma_{2}^{2}}-i \eta(r-q)+\Theta_{1}+\Theta_{2}\right] \tau\right\} \\
& \quad \times \Gamma\left(\frac{2 \Phi_{1}}{\sigma_{1}^{2}}-1\right)\left[1-\Gamma\left(\frac{2 \Phi_{1}}{\sigma_{1}^{2}}-1 ; \frac{4 \Omega_{1}^{2} v_{1,0} z_{1}\left(z_{1}-1\right)}{\sigma_{1}^{2}\left[\sigma_{1}^{2} s_{1}-\Theta_{1}+\Omega_{1}+2 \Omega_{1}\left(z_{1}-1\right)\right]}\right)\right] .
\end{aligned}
$$

The third component may be represented as

$$
\begin{aligned}
J_{3}=\frac{1}{\Omega_{2}} \int_{z_{2}}^{\infty} f_{2}\left(t, s_{1}\right) \exp \left\{\left[\frac{\left(\Phi_{1}-\sigma_{1}^{2}\right)\left(\Theta_{1}-\Omega_{1}\right)}{\sigma_{1}^{2}}+\frac{\left(\Phi_{2}-\sigma_{2}^{2}\right)\left(\Theta_{2}-\Omega_{2}\right)}{\sigma_{2}^{2}}-i \eta(r-q)+\Theta_{1}+\Theta_{2}\right](\tau-t)\right\} \\
\quad \times\left(\frac{2 \Omega_{1} \zeta_{1}\left(z_{1}-1\right)}{\left(\sigma_{1}^{2} s_{1}-\Theta_{1}+\Omega_{1}\right)\left(\zeta_{1}-z_{1}\right)+2 \Omega_{1} \zeta_{1}\left(z_{1}-1\right)}\right)^{\frac{2}{\sigma_{1}^{2}}\left(\sigma_{1}^{2}-\Phi_{1}\right)} \\
\quad \times\left(\frac{2 \Omega_{2} \zeta_{2}\left(z_{2}-1\right)}{\left(\sigma_{2}^{2} s_{2}-\Theta_{2}+\Omega_{2}\right)\left(\zeta_{2}-z_{2}\right)+2 \Omega_{2} \zeta_{2}\left(z_{2}-1\right)}\right)^{\frac{2}{\sigma_{2}^{2}}\left(\sigma_{2}^{2}-\Phi_{2}\right)} \frac{d \zeta_{2}}{\zeta_{2}\left(\zeta_{2}-1\right)} .
\end{aligned}
$$

Now by substituting the value of $f_{2}\left(t, s_{1}\right)$ in equation (A4.46) into equation (A4.58) we obtain

$$
\begin{aligned}
J_{3}= & \frac{-1}{\Gamma\left(\frac{2 \Phi_{2}}{\sigma_{2}^{2}}-1\right)} \int_{z_{2}}^{\infty}\left(\frac{2 \Omega_{2} v_{2,0}}{\sigma_{2}^{2}}\right)^{\frac{2 \Phi_{2}}{\sigma_{2}^{2}}-1} \exp \left\{-\left(\frac{\Theta_{2}-\Omega_{2}}{\sigma_{2}^{2}}+\frac{2 \Omega_{2} \zeta_{2}}{\sigma_{2}^{2}}\right) v_{2,0}+i \eta x_{0}\right\} \\
& \times \exp \left\{-\left(\frac{\Theta_{1}-\Omega_{1}}{\sigma_{1}^{2}}+\frac{\left(\sigma_{1}^{2} s_{1}-\Theta_{1}+\Omega_{1}\right) 2 \Omega_{1} z_{1}}{\left[\sigma_{1}^{2} s_{1}-\Theta_{1}+\Omega_{1}+2 \Omega_{1}\left(z_{1}-1\right)\right] \sigma_{1}^{2}}\right) v_{1,0}\right\} \\
& \times \exp \left\{\left[\frac{\left(\Phi_{1}-\sigma_{1}^{2}\right)\left(\Theta_{1}-\Omega_{1}\right)}{\sigma_{1}^{2}}+\frac{\left(\Phi_{2}-\sigma_{2}^{2}\right)\left(\Theta_{2}-\Omega_{2}\right)}{\sigma_{2}^{2}}-i \eta(r-q)+\Theta_{1}+\Theta_{2}\right] \tau\right\} \\
& \times\left(\frac{2 \Omega_{1}\left(z_{1}-1\right)}{\sigma_{1}^{2} s_{1}-\Theta_{1}+\Omega_{1}+2 \Omega_{1}\left(z_{1}-1\right)}\right)^{\frac{2}{\sigma_{2}^{2}}\left(\sigma_{1}^{2}-\Phi_{2}\right)} d \zeta_{2} . \\
& \times\left(\frac{2 \Omega_{2}\left(z_{2}-1\right)}{\left(\sigma_{2}^{2} s_{2}-\Theta_{2}+\Omega_{2}\right)\left(\zeta_{2}-z_{2}\right)+2 \Omega_{2} \zeta_{2}\left(z_{2}-1\right)}\right)^{\text {(A4.59) }}
\end{aligned}
$$

By proceeding as we did when handling the $J_{2}$ term in equation (A4.51) it can be shown that 


$$
\begin{aligned}
J_{3}= & \frac{-\left[2 \Omega_{2}\left(z_{2}-1\right)\right]^{2-\frac{2 \Phi_{2}}{\sigma_{2}^{2}}}}{\Gamma\left(\frac{2 \Phi_{2}}{\sigma_{2}^{2}}-1\right)}\left(\frac{2 \Omega_{2} v_{2,0}}{\sigma_{2}^{2}}\right)^{\frac{2 \Phi_{2}}{\sigma_{2}^{2}}-1} \exp \left\{-\left(\frac{\Theta_{2}-\Omega_{2}}{\sigma_{2}^{2}}\right) v_{2,0}+i \eta x_{0}\right\} \\
& \times \exp \left\{-\left(\frac{\Theta_{1}-\Omega_{1}}{\sigma_{1}^{2}}+\frac{\left(\sigma_{1}^{2} s_{1}-\Theta_{1}+\Omega_{1}\right) 2 \Omega_{1} z_{1}}{\left[\sigma_{1}^{2} s_{1}-\Theta_{1}+\Omega_{1}+2 \Omega_{1}\left(z_{1}-1\right)\right] \sigma_{1}^{2}}\right) v_{1,0}\right\} \\
& \times \exp \left\{\left[\frac{\left(\Phi_{1}-\sigma_{1}^{2}\right)\left(\Theta_{1}-\Omega_{1}\right)}{\sigma_{1}^{2}}+\frac{\left(\Phi_{2}-\sigma_{2}^{2}\right)\left(\Theta_{2}-\Omega_{2}\right)}{\sigma_{2}^{2}}-i \eta(r-q)+\Theta_{1}+\Theta_{2}\right] \tau\right\} \\
& \times\left(\frac{2 \Omega_{1}\left(z_{1}-1\right)}{\sigma_{1}^{2} s_{1}-\Theta_{1}+\Omega_{1}+2 \Omega_{1}\left(z_{1}-1\right)}\right)^{\frac{2}{\sigma_{1}^{2}}\left(\sigma_{1}^{2}-\Phi_{1}\right)} G_{2}\left(v_{2,0}\right),
\end{aligned}
$$

where

$$
G_{2}\left(v_{2}\right)=\int_{z_{2}}^{\infty} e^{-\frac{2 \Omega_{2} v_{2}}{\sigma_{2}^{2}} \zeta_{2}}\left[\left(\sigma_{2}^{2} s_{2}-\Theta_{2}+\Omega_{2}\right)\left(\zeta_{2}-z_{2}\right)+2 \Omega_{2} \zeta_{2}\left(z_{2}-1\right)\right]^{\frac{2 \Phi_{2}}{\sigma_{2}^{2}}-2} d \zeta_{2}
$$

Simplifying $G_{2}\left(v_{2}\right)$ in an analogous fashion to the way $G_{1}\left(v_{1}\right)$ was simplified we obtain

$$
\begin{aligned}
G_{2}\left(v_{2}\right) & =\left[\sigma_{2}^{2} s_{2}-\Theta_{2}+\Omega_{2}+2 \Omega_{2}\left(z_{2}-1\right)\right]^{\frac{2 \Phi_{2}}{\sigma_{2}^{2}}-2}\left(\frac{\sigma_{2}^{2}}{2 \Omega_{2} v_{2}}\right)^{\frac{2 \Phi_{2}}{\sigma_{2}^{2}}-1} \exp \left\{\frac{-2 \Omega_{2} v_{2}\left(\sigma_{2}^{2} s_{2}-\Theta_{2}+\Omega_{2}\right) z_{2}}{\sigma_{2}^{2}\left[\sigma_{2}^{2} s_{2}-\Theta_{2}+\Omega_{2}+2 \Omega_{2}\left(z_{2}-1\right)\right]}\right\} \\
& \times\left[\Gamma\left(\frac{2 \Phi_{2}}{\sigma_{2}^{2}}-1\right)-\int_{0}^{\frac{4 \Omega_{2}^{2} v_{2} z_{2}\left(z_{2}-1\right)}{\sigma_{2}^{2}\left[\sigma_{2}^{2} s_{2}-\Theta_{2}+\Omega_{2}+2 \Omega_{2}\left(z_{2}-1\right)\right]}} e^{-\xi_{2}} \xi_{2}^{\left(\frac{2 \Omega_{2}}{\sigma_{2}^{2}}-1\right)-1} d \xi_{2}\right] .
\end{aligned}
$$

Substituting equation (A4.62) into equation (A4.60) we obtain

$$
\begin{aligned}
J_{3} & =\frac{-1}{\Gamma\left(\frac{2 \Phi_{2}}{\sigma_{2}^{2}}-1\right)}\left(\frac{2 \Omega_{1}\left(z_{1}-1\right)}{\sigma_{1}^{2} s_{1}-\Theta_{1}+\Omega_{1}+2 \Omega_{1}\left(z_{1}-1\right)}\right)^{2-\frac{2 \Phi_{1}}{\sigma_{1}^{2}}}\left(\frac{2 \Omega_{2}\left(z_{2}-1\right)}{\sigma_{2}^{2} s_{2}-\Theta_{2}+\Omega_{2}+2 \Omega_{2}\left(z_{2}-1\right)}\right)^{2-\frac{2 \Phi_{2}}{\sigma_{2}^{2}}} \\
& \times \exp \left\{-\left(\frac{\Theta_{1}-\Omega_{1}}{\sigma_{1}^{2}}\right) v_{1,0}-\left(\frac{\Theta_{2}-\Omega_{2}}{\sigma_{2}^{2}}\right) v_{2,0}+i \eta x_{0}\right\} \\
& \times \exp \left\{\frac{-2 \Omega_{1} v_{1,0}\left(\sigma_{1}^{2} s_{1}-\Theta_{1}+\Omega_{1}\right) z_{1}}{\sigma_{1}^{2} s_{1}-\Theta_{1}+\Omega_{1}+2 \Omega_{1}\left(z_{1}-1\right)}\right\} \exp \left\{\frac{-2 \Omega_{2} v_{2,0}\left(\sigma_{2}^{2} s_{2}-\Theta_{2}+\Omega_{2}\right) z_{2}}{\left[\sigma_{2}^{2} s_{2}-\Theta_{2}+\Omega_{2}+2 \Omega_{2}\left(z_{2}-1\right)\right] \sigma_{2}^{2}}\right\} \\
& \times \exp \left\{\left[\frac{\left(\Phi_{1}-\sigma_{1}^{2}\right)\left(\Theta_{1}-\Omega_{1}\right)}{\sigma_{1}^{2}}+\frac{\left(\Phi_{2}-\sigma_{2}^{2}\right)\left(\Theta_{2}-\Omega_{2}\right)}{\sigma_{2}^{2}}-i \eta(r-q)+\Theta_{1}+\Theta_{2}\right] \tau\right\} \\
& \times \Gamma\left(\frac{2 \Phi_{2}}{\sigma_{2}^{2}}-1\right)\left[1-\Gamma\left(\frac{2 \Phi_{2}}{\sigma_{2}^{2}}-1 ; \frac{4 \Omega_{2}^{2} v_{2,0} z_{2}\left(z_{2}-1\right)}{\sigma_{2}^{2}\left[\sigma_{2}^{2} s_{2}-\Theta_{2}+\Omega_{2}+2 \Omega_{2}\left(z_{2}-1\right)\right]}\right] .\right.
\end{aligned}
$$


By combining $J_{1}, J_{2}$ and $J_{3}$ equation (A4.27) becomes

$$
\begin{aligned}
& \tilde{U}\left(\tau, \eta, s_{1}, s_{2}\right)=\left(\frac{2 \Omega_{1}\left(z_{1}-1\right)}{\sigma_{1}^{2} s_{1}-\Theta_{1}+\Omega_{1}+2 \Omega_{1}\left(z_{1}-1\right)}\right)^{2-\frac{2 \Phi_{1}}{\sigma_{1}^{2}}}\left(\frac{2 \Omega_{2}\left(z_{2}-1\right)}{\sigma_{2}^{2} s_{2}-\Theta_{2}+\Omega_{2}+2 \Omega_{2}\left(z_{2}-1\right)}\right)^{2-\frac{2 \Phi_{2}}{\sigma_{2}^{2}}} \\
& \times \exp \left\{-\left(\frac{\Theta_{1}-\Omega_{1}}{\sigma_{1}^{2}}\right) v_{1,0}-\left(\frac{\Theta_{2}-\Omega_{2}}{\sigma_{2}^{2}}\right) v_{2,0}+i \eta x_{0}\right\} \\
& \times \exp \left\{\left[\frac{\left(\Phi_{1}-\sigma_{1}^{2}\right)\left(\Theta_{1}-\Omega_{1}\right)}{\sigma_{1}^{2}}+\frac{\left(\Phi_{2}-\sigma_{2}^{2}\right)\left(\Theta_{2}-\Omega_{2}\right)}{\sigma_{2}^{2}}-i \eta(r-q)+\Theta_{1}+\Theta_{2}\right] \tau\right\} \\
& \times \exp \left\{\frac{-2 \Omega_{1} v_{1,0}\left(\sigma_{1}^{2} s_{1}-\Theta_{1}+\Omega_{1}\right) z_{1}}{\sigma_{1}^{2}\left[\sigma_{1}^{2} s_{1}-\Theta_{1}+\Omega_{1}+2 \Omega_{1}\left(z_{1}-1\right)\right]}\right\} \exp \left\{\frac{-2 \Omega_{2} v_{2,0}\left(\sigma_{2}^{2} s_{2}-\Theta_{2}+\Omega_{2}\right) z_{2}}{\left[\sigma_{2}^{2} s_{2}-\Theta_{2}+\Omega_{2}+2 \Omega_{2}\left(z_{2}-1\right)\right] \sigma_{2}^{2}}\right\} \\
& \times {\left[\Gamma\left(\frac{2 \Phi_{1}}{\sigma_{1}^{2}}-1 ; \frac{4 \Omega_{1}^{2} v_{1,0} z_{1}\left(z_{1}-1\right)}{\sigma_{1}^{2}\left[\sigma_{1}^{2} s_{1}-\Theta_{1}+\Omega_{1}+2 \Omega_{1}\left(z_{1}-1\right)\right]}\right)\right.} \\
&\left.+\Gamma\left(\frac{2 \Phi_{2}}{\sigma_{2}^{2}}-1 ; \frac{4 \Omega_{2}^{2} v_{2,0} z_{2}\left(z_{2}-1\right)}{\sigma_{2}^{2}\left[\sigma_{2}^{2} s_{2}-\Theta_{2}+\Omega_{2}+2 \Omega_{2}\left(z_{2}-1\right)\right]}\right)-1\right] .
\end{aligned}
$$

We recall from equation (A4.30) that $z_{1}^{-1}=1-e^{-\Omega_{1} \tau}$ and $z_{2}^{-1}=1-e^{-\Omega_{2} \tau}$ where $\Omega_{1}$ and $\Omega_{2}$ have been defined in equations (A4.3) and (A4.8) respectively. Substituting these expressions into the above equation we finally obtain

$$
\begin{aligned}
& \tilde{U}\left(\tau, \eta, s_{1}, s_{2}\right)=\left(\frac{2 \Omega_{1}}{\left(\sigma_{1}^{2} s_{1}-\Theta_{1}+\Omega_{1}\right)\left(e^{\Omega_{1} \tau}-1\right)+2 \Omega_{1}}\right)^{2-\frac{2 \Phi_{1}}{\sigma_{1}^{2}}}\left(\frac{2 \Omega_{2}}{\left(\sigma_{2}^{2} s_{2}-\Theta_{2}+\Omega_{2}\right)\left(e^{\Omega_{2} \tau}-1\right)+2 \Omega_{2}}\right)^{2-\frac{2 \Phi_{2}}{\sigma_{2}^{2}}} \\
& \times \exp \left\{-\left(\frac{\Theta_{1}-\Omega_{1}}{\sigma_{1}^{2}}\right) v_{1,0}-\left(\frac{\Theta_{2}-\Omega_{2}}{\sigma_{2}^{2}}\right) v_{2,0}+i \eta x_{0}\right\} \\
& \times \exp \left\{\left[\frac{\left(\Phi_{1}-\sigma_{1}^{2}\right)\left(\Theta_{1}-\Omega_{1}\right)}{\sigma_{1}^{2}}+\frac{\left(\Phi_{2}-\sigma_{2}^{2}\right)\left(\Theta_{2}-\Omega_{2}\right)}{\sigma_{2}^{2}}-i \eta(r-q)+\Theta_{1}+\Theta_{2}\right] \tau\right\} \\
& \times \exp \left\{\frac{-2 \Omega_{1} v_{1,0}\left(\sigma_{1}^{2} s_{1}-\Theta_{1}+\Omega_{1}\right) e^{\Omega_{1} \tau}}{\sigma_{1}^{2}\left[\left(\sigma_{1}^{2} s_{1}-\Theta_{1}+\Omega_{1}\right)\left(e^{\Omega_{1} \tau}-1\right)+2 \Omega_{1}\right]}\right\} \exp \left\{\frac{-2 \Omega_{2} v_{2,0}\left(\sigma_{2}^{2} s_{2}-\Theta_{2}+\Omega_{2}\right) e^{\Omega_{2} \tau}}{\sigma_{2}^{2}\left[\left(\sigma_{2}^{2} s_{2}-\Theta_{2}+\Omega_{2}\right)\left(e^{\Omega_{2} \tau}-1\right)+2 \Omega_{2}\right]}\right\} \\
& \times {\left[\Gamma\left(\frac{2 \Phi_{1}}{\sigma_{1}^{2}}-1 ; \frac{2 \Omega_{1} v_{1,0} e^{\Omega_{1} \tau}}{\sigma_{1}^{2}\left(e^{\Omega_{1} \tau}-1\right)} \times \frac{2 \Omega_{1}}{\left(\sigma_{1}^{2} s_{1}-\Theta_{1}+\Omega_{1}\right)\left(e^{\Omega_{1} \tau}-1\right)+2 \Omega_{1}}\right)\right.} \\
&\left.+\Gamma\left(\frac{2 \Phi_{2}}{\sigma_{2}^{2}}-1 ; \frac{2 \Omega_{2} v_{2,0} e^{\Omega_{2} \tau}}{\sigma_{2}^{2}\left(e^{\Omega_{2} \tau}-1\right)} \times \frac{2 \Omega_{2}}{\left(\sigma_{2}^{2} s_{2}-\Theta_{2}+\Omega_{2}\right)\left(e^{\Omega_{2} \tau}-1\right)+2 \Omega_{2}}\right)-1\right],
\end{aligned}
$$

which is the result presented in Proposition 7.1.

\section{Appendix 5. Proof of Proposition 8.1}

Our calculations are facilitated by carrying out the transformations

$$
\left\{\begin{array}{l}
A_{1}=\frac{2 \Omega_{1} v_{1,0}}{\sigma_{1}^{2}\left(1-e^{-\Omega_{1} \tau}\right)}, \\
A_{2}=\frac{2 \Omega_{2} v_{2,0}}{\sigma_{2}^{2}\left(1-e^{-\Omega_{2} \tau}\right)},
\end{array}\right.
$$




$$
\left\{\begin{array}{l}
z_{1}=\frac{\left(\sigma_{1}^{2} s_{1}-\Theta_{1}+\Omega_{1}\right)\left(e^{\Omega_{1} \tau}-1\right)+2 \Omega_{1}}{2 \Omega_{1}}, \\
z_{2}=\frac{\left(\sigma_{2}^{2} s_{2}-\Theta_{2}+\Omega_{2}\right)\left(e_{2} \tau-1\right)+2 \Omega_{2}}{2 \Omega_{2}},
\end{array}\right.
$$

and

$$
\begin{aligned}
h\left(\tau, \eta, v_{1,0}, v_{2,0}\right)= & \exp \left\{\left[\frac{\left(\Phi_{1}-\sigma_{1}^{2}\right)\left(\Theta_{1}-\Omega_{1}\right)}{\sigma_{1}^{2}}+\frac{\left(\Phi_{2}-\sigma_{2}^{2}\right)\left(\Theta_{2}-\Omega_{2}\right)}{\sigma_{2}^{2}}-i \eta(r-q)+\Theta_{1}+\Theta_{2}\right] \tau\right\} \\
& \times \exp \left\{-\left(\frac{\Theta_{1}-\Omega_{1}}{\sigma_{1}^{2}}\right) v_{1,0}-\left(\frac{\Theta_{2}-\Omega_{2}}{\sigma_{2}^{2}}\right) v_{2,0}+i \eta x_{0}\right\} .
\end{aligned}
$$

Substituting these into equation (7.1) we obtain ${ }^{13}$

$$
\begin{aligned}
\tilde{U}\left(\tau, \eta, s_{1}\left(z_{1}\right),\right. & \left.s_{2}\left(z_{2}\right)\right)=h\left(\tau, \eta, v_{1,0}, v_{2,0}\right) z_{1}^{\frac{2 \Phi_{1}}{\sigma_{1}^{2}}-2} z_{2}^{\frac{2 \Phi_{2}}{\sigma_{2}^{2}}-2} \exp \left\{-\frac{A_{1}}{z_{1}}\left(z_{1}-1\right)\right\} \exp \left\{-\frac{A_{2}}{z_{2}}\left(z_{2}-1\right)\right\} \\
& \times\left[\Gamma\left(\frac{2 \Phi_{1}}{\sigma_{1}^{2}}-1 ; \frac{A_{1}}{z_{1}}\right)+\Gamma\left(\frac{2 \Phi_{2}}{\sigma_{2}^{2}}-1 ; \frac{A_{2}}{z_{2}}\right)-1\right] \\
& =h\left(\tau, \eta, v_{1,0}, v_{2,0}\right) z_{1}^{\frac{2 \Phi_{1}}{\sigma_{1}^{2}}-2} z_{2}^{\frac{2 \Phi_{2}}{\sigma_{2}^{2}}-2} \exp \left\{-\frac{A_{1}}{z_{1}}\left(z_{1}-1\right)\right\} \exp \left\{-\frac{A_{2}}{z_{2}}\left(z_{2}-1\right)\right\} \\
& \times\left[\frac{1}{\Gamma\left(\frac{2 \Phi_{1}}{\sigma_{1}^{2}}-1\right)} \int_{0}^{\frac{A_{1}}{z_{1}}} e^{-\beta_{1}} \beta_{1}^{\frac{2 \Phi_{1}}{\sigma_{1}^{2}}-2} d \beta_{1}+\frac{1}{\Gamma\left(\frac{2 \Phi_{2}}{\sigma_{2}^{2}}-1\right)} \int_{0}^{\frac{A_{2}}{z_{2}}} e^{-\beta_{2}} \beta_{2}^{\frac{2 \Phi_{2}}{\sigma_{2}^{2}}-2} d \beta_{2}-1\right] .
\end{aligned}
$$

In order to evaluate equation (A5.4), we break it into three parts such that

$$
\tilde{U}\left(\tau, \eta, s_{1}\left(z_{1}\right), s_{2}\left(z_{2}\right)\right)=\tilde{F}_{1}\left(\tau, \eta, s_{1}\left(z_{1}\right), s_{2}\left(z_{2}\right)\right)+\tilde{F}_{2}\left(\tau, \eta, s_{1}\left(z_{1}\right), s_{2}\left(z_{2}\right)\right)+\tilde{F}_{3}\left(\tau, \eta, s_{1}\left(z_{1}\right), s_{2}\left(z_{2}\right)\right),
$$

where

$$
\begin{aligned}
\tilde{F}_{1}\left(\tau, \eta, s_{1}\left(z_{1}\right), s_{2}\left(z_{2}\right)\right) & =h\left(\tau, \eta, v_{1,0}, v_{2,0}\right) z_{1}^{\frac{2 \Phi_{1}}{\sigma_{1}^{1}}-2} z_{2}^{\frac{2 \Phi_{2}}{\sigma_{2}^{2}}-2} \exp \left\{-\frac{A_{1}}{z_{1}}\left(z_{1}-1\right)\right\} \exp \left\{-\frac{A_{2}}{z_{2}}\left(z_{2}-1\right)\right\} \\
& \times \frac{1}{\Gamma\left(\frac{2 \Phi_{1}}{\sigma_{1}^{2}}-1\right)} \int_{0}^{\frac{A_{1}}{z_{1}}} e^{-\beta_{1}} \beta_{1}^{\frac{2 \Phi_{1}}{\sigma_{1}^{2}}-2} d \beta_{1}, \\
\tilde{F}_{2}\left(\tau, \eta, s_{1}\left(z_{1}\right), s_{2}\left(z_{2}\right)\right) & =h\left(\tau, \eta, v_{1,0}, v_{2,0}\right) z_{1}^{\frac{2 \Phi_{1}}{\sigma_{1}^{2}}-2} z_{2}^{\frac{2 \Phi_{2}}{\sigma_{2}^{2}}-2} \exp \left\{-\frac{A_{1}}{z_{1}}\left(z_{1}-1\right)\right\} \exp \left\{-\frac{A_{2}}{z_{2}}\left(z_{2}-1\right)\right\} \\
& \times \frac{1}{\Gamma\left(\frac{2 \Phi_{2}}{\sigma_{2}^{2}}-1\right)} \int_{0}^{\frac{A_{2}}{z_{2}}} e^{-\beta_{2}} \beta_{2}^{\frac{2 \Phi_{2}}{\sigma_{2}^{2}}-2} d \beta_{2},
\end{aligned}
$$

\footnotetext{
${ }^{13}$ Note that the system (A5.2) defines $s_{1}$ as a function of $z_{1}$ and $s_{2}$ as a function of $z_{2}$.
} 
and

$\tilde{F}_{3}\left(\tau, \eta, s_{1}\left(z_{1}\right), s_{2}\left(z_{2}\right)\right)=-h\left(\tau, \eta, v_{1,0}, v_{2,0}\right) z_{1}^{\frac{2 \Phi_{1}}{\sigma_{1}^{2}}-2} z_{2}^{\frac{2 \Phi_{2}}{\sigma_{2}^{2}}-2} \exp \left\{-\frac{A_{1}}{z_{1}}\left(z_{1}-1\right)\right\} \exp \left\{-\frac{A_{2}}{z_{2}}\left(z_{2}-1\right)\right\}$.

Simplifying the $\tilde{\mathbf{F}}_{\mathbf{1}}\left(\tau, \eta, \mathbf{s}_{\mathbf{1}}\left(\mathbf{z}_{1}\right), \mathbf{s}_{\mathbf{2}}\left(\mathbf{z}_{2}\right)\right)$ term:

The first term is simplified by first setting $\xi_{1}=1-\frac{z_{1}}{A_{1}} \beta_{1}$ in equation (A5.6) to obtain

$$
\begin{aligned}
\tilde{F}_{1}\left(\tau, \eta, s_{1}\left(z_{1}\right), s_{2}\left(z_{2}\right)\right) & =h\left(\tau, \eta, v_{1,0}, v_{2,0}\right) e^{-\left(A_{1}+A_{2}\right)} \frac{A_{1}^{\frac{2 \Phi_{1}}{\sigma_{1}^{2}}-1}}{\Gamma\left(\frac{2 \Phi_{1}}{\sigma_{1}^{2}}-1\right)} e^{\frac{A_{2}}{z_{2}}} z_{2}^{\frac{2 \Phi_{2}}{\sigma_{2}^{2}}-2} \\
& \times \int_{0}^{1}\left(1-\xi_{1}\right)^{\frac{2 \Phi_{1}}{\sigma_{1}^{2}}-2} z_{1}^{-1} e^{\frac{A_{1}}{z_{1}} \xi_{1}} d \xi_{1}
\end{aligned}
$$

From the transformation (A5.2) we can express the Laplace transform variables as

$$
s_{1}=\frac{2 \Omega_{1}\left(z_{1}-1\right)}{\sigma_{1}^{2}\left(e^{\Omega_{1} \tau}-1\right)}+\frac{\Theta_{1}-\Omega_{1}}{\sigma_{1}^{2}}, \quad s_{2}=\frac{2 \Omega_{2}\left(z_{2}-1\right)}{\sigma_{2}^{2}\left(e^{\Omega_{2} \tau}-1\right)}+\frac{\Theta_{2}-\Omega_{2}}{\sigma_{2}^{2}},
$$

Using the definition of Laplace transforms provided in equation (4.3), we can represent the above transform as

$$
\begin{aligned}
\mathcal{L}\left\{\hat{F}_{1}\left(\tau, \eta, v_{1}, v_{2}\right)\right\} & =\int_{0}^{\infty} \int_{0}^{\infty} \exp \left\{-\left[\frac{2 \Omega_{1}\left(z_{1}-1\right)}{\sigma_{1}^{2}\left(e^{\Omega_{1} \tau}-1\right)}+\frac{\Theta_{1}-\Omega_{1}}{\sigma_{1}^{2}}\right] v_{1}-\left[\frac{2 \Omega_{2}\left(z_{2}-1\right)}{\sigma_{2}^{2}\left(e^{\Omega_{2} \tau}-1\right)}+\frac{\Theta_{2}-\Omega_{2}}{\sigma_{2}^{2}}\right] v_{2}\right\} \\
& \times \hat{F}_{1}\left(\tau, \eta, v_{1}, v_{2}\right) d v_{1} d v_{2} .
\end{aligned}
$$

Making the change of variables

$$
y_{1}=\frac{2 \Omega_{1} v_{1}}{\sigma_{1}^{2}\left(e^{\Omega_{1} \tau}-1\right)} \quad \text { and } \quad y_{2}=\frac{2 \Omega_{2} v_{2}}{\sigma_{2}^{2}\left(e^{\Omega_{2} \tau}-1\right)}
$$

in equation (A5.10) and rearranging we obtain

$$
\begin{gathered}
\mathcal{L}\left\{\hat{F}_{1}\left(\tau, \eta, v_{1}\left(y_{1}\right), v_{2}\left(y_{2}\right)\right)\right\}=\frac{\sigma_{1}^{2}\left(e^{\Omega_{1} \tau}-1\right)}{2 \Omega_{1}} \frac{\sigma_{2}^{2}\left(e^{\Omega_{2} \tau}-1\right)}{2 \Omega_{2}} \int_{0}^{\infty} \int_{0}^{\infty} e^{-z_{1} y_{1}-z_{2} y_{2}} \\
\times \exp \left(-\left[\frac{\left(\Theta_{1}-\Omega_{1}\right)\left(e^{\Omega_{1} \tau}-1\right)}{2 \Omega_{1}}-1\right] y_{1}\right) \exp \left(-\left[\frac{\left(\Theta_{2}-\Omega_{2}\right)\left(e^{\Omega_{2} \tau}-1\right)}{2 \Omega_{2}}-1\right] y_{2}\right) \\
\times \hat{F}_{1}\left(\tau, \eta, v_{1}\left(y_{1}\right), v_{2}\left(y_{2}\right)\right) d y_{1} d y_{2} .
\end{gathered}
$$

The Laplace transform of the RHS of equation (A5.12) can also be represented as

$$
\begin{aligned}
& \mathcal{L}\left\{\hat{F}_{1}\left(\tau, \eta, v_{1}\left(y_{1}\right), v_{2}\left(y_{2}\right)\right)\right\}=\frac{\sigma_{1}^{2}\left(e^{\Omega_{1} \tau}-1\right)}{2 \Omega_{1}} \frac{\sigma_{2}^{2}\left(e^{\Omega_{2} \tau}-1\right)}{2 \Omega_{2}} \mathcal{L}\left\{\exp \left(-\left[\frac{\left(\Theta_{1}-\Omega_{1}\right)\left(e^{\Omega_{1} \tau}-1\right)}{2 \Omega_{1}}-1\right] y_{1}\right)\right. \\
& \left.\quad \times \exp \left(-\left[\frac{\left(\Theta_{2}-\Omega_{2}\right)\left(e^{\Omega_{2} \tau}-1\right)}{2 \Omega_{2}}-1\right] y_{2}\right) \hat{F}_{1}\left(\tau, \eta, v_{1}\left(y_{1}\right), v_{2}\left(y_{2}\right)\right)\right\} .
\end{aligned}
$$


Recalling that

$$
\mathcal{L}\left\{\hat{F}_{1}\left(\tau, \eta, y_{1}, y_{2}\right)\right\}=\int_{0}^{\infty} \int_{0}^{\infty} e^{-z_{1} y_{1}-z_{2} y_{2}} \hat{F}_{1}\left(\tau, \eta, y_{1}, y_{2}\right) d y_{1} d y_{2}=\tilde{F}\left(\tau, \eta, z_{1}, z_{2}\right),
$$

equation (A5.13) can be written in terms of the inverse Laplace transform as

$$
\begin{gathered}
\mathcal{L}^{-1}\left\{\tilde{F}_{1}\left(\tau, \eta, s_{1}\left(z_{1}\right), s_{2}\left(z_{2}\right)\right)\right\}=\frac{2 \Omega_{1}}{\sigma_{1}^{2}\left(e^{\Omega_{1} \tau}-1\right)} \frac{2 \Omega_{2}}{\sigma_{2}^{2}\left(e^{\Omega_{2} \tau}-1\right)} \exp \left(\left[\frac{\left(\Theta_{1}-\Omega_{1}\right)\left(e^{\Omega_{1} \tau}-1\right)}{2 \Omega_{1}}-1\right] y_{1}\right) \\
\times \exp \left(\left[\frac{\left(\Theta_{2}-\Omega_{2}\right)\left(e^{\Omega_{2} \tau}-1\right)}{2 \Omega_{2}}-1\right] y_{2}\right) \mathcal{L}^{-1}\left\{\tilde{F}_{1}\left(\tau, \eta, s_{1}\left(z_{1}\right), s_{2}\left(z_{2}\right)\right)\right\}
\end{gathered}
$$

Applying the inverse transform (A5.15) to equation (A5.9) we obtain

$$
\begin{gathered}
\hat{F}_{1}\left(\tau, \eta, v_{1}\left(y_{1}\right), v_{2}\left(y_{2}\right)\right)=h\left(\tau, \eta, v_{1,0}, v_{2,0}\right) e^{-\left(A_{1}+A_{2}\right)} \frac{A_{1}^{\frac{2 \Phi_{1}}{\sigma_{1}^{2}}-1}}{\Gamma\left(\frac{2 \Phi_{1}}{\sigma_{1}^{2}}-1\right)} \frac{2 \Omega_{1}}{\sigma_{1}^{2}\left(e^{\Omega_{1} \tau}-1\right)} \frac{2 \Omega_{2}}{\sigma_{2}^{2}\left(e^{\Omega_{2} \tau}-1\right)} \\
\times \exp \left\{\left[\frac{\left(\Theta_{1}-\Omega_{1}\right)\left(e^{\Omega_{1} \tau}-1\right)}{2 \Omega_{1}}-1\right] y_{1}\right\} \exp \left\{\left[\frac{\left(\Theta_{2}-\Omega_{2}\right)\left(e^{\Omega_{2} \tau}-1\right)}{2 \Omega_{2}}-1\right] y_{2}\right\} \\
\times \int_{0}^{1}\left(1-\xi_{1}\right)^{\frac{2 \Phi_{1}}{\sigma_{1}^{2}}-2} \mathcal{L}^{-1}\left\{z_{2}^{\frac{2 \Phi_{2}}{\sigma_{2}^{2}}-2} e^{\left.\frac{A_{2}}{z_{2}} z_{1}^{-1} e^{\frac{A_{1}}{z_{1}} \xi_{1}}\right\} d \xi_{1} .}\right.
\end{gathered}
$$

From Abramowitz and Stegun (1964) $)^{14}$ we find that

$$
\mathcal{L}\left\{\left(\frac{y_{1}}{A_{1}}\right)^{\frac{u_{1}-1}{2}} I_{u_{1}-1}\left(2 \sqrt{A_{1} y_{1}}\right)\left(\frac{y_{2}}{A_{2}}\right)^{\frac{u_{2}-1}{2}} I_{u_{2}-1}\left(2 \sqrt{A_{2} y_{2}}\right)\right\}=\frac{1}{z_{1}^{u_{1}}} e^{\frac{A_{1}}{z_{1}}} \frac{1}{z_{2}^{u_{2}}} e^{\frac{A_{2}}{z_{2}}}
$$

where, $I_{k}(z)$ is the modified Bessel function of the first kind defined as

$$
I_{k}(z)=\sum_{n=0}^{\infty} \frac{\left(\frac{z}{2}\right)^{2 n+k}}{\Gamma(n+k+1) n !}
$$

Application of the result in equation (A5.17) to equation (A5.16) yields

$$
\begin{aligned}
\hat{F}_{1}(\tau, \eta, & \left.v_{1}\left(y_{1}\right), v_{2}\left(y_{2}\right)\right)=h\left(\tau, \eta, v_{1,0}, v_{2,0}\right) e^{-\left(A_{1}+A_{2}\right)} \frac{2 \Omega_{1}}{\sigma_{1}^{2}\left(e^{\Omega_{1} \tau}-1\right)} \frac{2 \Omega_{2}}{\sigma_{2}^{2}\left(e^{\Omega_{2} \tau}-1\right)} \\
& \times \exp \left\{\left[\frac{\left(\Theta_{1}-\Omega_{1}\right)\left(e^{\Omega_{1} \tau}-1\right)}{2 \Omega_{1}}-1\right] y_{1}\right\} \exp \left\{\left[\frac{\left(\Theta_{2}-\Omega_{2}\right)\left(e^{\Omega_{2} \tau}-1\right)}{2 \Omega_{2}}-1\right] y_{2}\right\} \\
& \times\left(\frac{y_{2}}{A_{2}}\right)^{\frac{2 \Phi_{1}}{\sigma^{2}-1}-\frac{\Phi_{2}}{\sigma_{2}^{2}}} I_{1-\frac{2 \Phi_{2}}{\sigma_{2}^{2}}}\left(2 \sqrt{A_{2} y_{2}}\right) \frac{A_{1}^{2}}{\Gamma\left(\frac{2 \Phi_{1}}{\sigma_{1}^{2}}-1\right)} \int_{0}^{1}\left(1-\xi_{1}\right)^{\frac{2 \Phi_{1}}{\sigma_{1}^{2}}-2} I_{0}\left(2 \sqrt{A_{1} y_{1} \xi_{1}}\right) d \xi_{1} .
\end{aligned}
$$

By expanding both terms inside the integral in power series followed by integration we find

\footnotetext{
${ }^{14}$ This result is tabulated on page 1026 of the referenced book.
} 
that

$$
\int_{0}^{1}\left(1-\xi_{1}\right)^{\frac{2 \Phi_{1}}{\sigma_{1}^{2}}-2} I_{0}\left(2 \sqrt{A_{1} y_{1} \xi_{1}}\right) d \xi_{1}=\Gamma\left(\frac{2 \Phi_{1}}{\sigma_{1}^{2}}-1\right)\left(A_{1} y_{1}\right)^{\frac{1}{2}-\frac{\Phi_{1}}{\sigma_{1}^{2}}} I_{\frac{2 \Phi_{1}}{\sigma_{1}^{2}}-1}\left(2 \sqrt{A_{1} y_{1}}\right) .
$$

Substituting this into equation (A5.19) we obtain the inverse Laplace transform of the first component of (A5.4) as ${ }^{15}$

$$
\begin{aligned}
\hat{F}_{1}\left(\tau, \eta, v_{1}\left(y_{1}\right), v_{2}\left(y_{2}\right)\right)=h\left(\tau, \eta, v_{1,0}, v_{2,0}\right) e^{-\left(A_{1}+A_{2}\right)} \frac{2 \Omega_{1}}{\sigma_{1}^{2}\left(e^{\Omega_{1} \tau}-1\right)} \frac{2 \Omega_{2}}{\sigma_{2}^{2}\left(e^{\Omega_{2} \tau}-1\right)} \\
\times \exp \left\{\left[\frac{\left(\Theta_{1}-\Omega_{1}\right)\left(e^{\Omega_{1} \tau}-1\right)}{2 \Omega_{1}}-1\right] y_{1}\right\} \exp \left\{\left[\frac{\left(\Theta_{2}-\Omega_{2}\right)\left(e^{\Omega_{2} \tau}-1\right)}{2 \Omega_{2}}-1\right] y_{2}\right\} \\
\times\left(\frac{A_{1}}{y_{1}}\right)^{\frac{\Phi_{1}}{\sigma_{1}^{2}}-\frac{1}{2}}\left(\frac{A_{2}}{y_{2}}\right)^{\frac{\Phi_{2}}{\sigma_{2}^{2}}-\frac{1}{2}} I_{\frac{2 \Phi_{1}}{\sigma_{1}^{2}}-1}\left(2 \sqrt{A_{1} y_{1}}\right) I_{\frac{2 \Phi_{2}}{\sigma_{2}^{2}}-1}\left(2 \sqrt{A_{2} y_{2}}\right)
\end{aligned}
$$

Simplifying the $\tilde{\mathbf{F}}_{\mathbf{2}}\left(\tau, \eta, \mathbf{s}_{\mathbf{1}}\left(\mathbf{z}_{\mathbf{1}}\right), \mathbf{s}_{\mathbf{2}}\left(\mathbf{z}_{\mathbf{2}}\right)\right)$ term:

By performing similar calculations to those outlined from (A5.9) - (A5.19) we find that

$$
\begin{gathered}
\hat{F}_{2}\left(\tau, \eta, v_{1}\left(y_{1}\right), v_{2}\left(y_{2}\right)\right)=h\left(\tau, \eta, v_{1,0}, v_{2,0}\right) e^{-\left(A_{1}+A_{2}\right)} \frac{2 \Omega_{1}}{\sigma_{1}^{2}\left(e^{\Omega_{1} \tau}-1\right)} \frac{2 \Omega_{2}}{\sigma_{2}^{2}\left(e^{\Omega_{2} \tau}-1\right)} \\
\times \exp \left\{\left[\frac{\left(\Theta_{1}-\Omega_{1}\right)\left(e^{\Omega_{1} \tau}-1\right)}{2 \Omega_{1}}-1\right] y_{1}\right\} \exp \left\{\left[\frac{\left(\Theta_{2}-\Omega_{2}\right)\left(e^{\Omega_{2} \tau}-1\right)}{2 \Omega_{2}}-1\right] y_{2}\right\} \\
\times\left(\frac{A_{1}}{y_{1}}\right)^{\frac{\Phi_{1}}{\sigma_{1}^{2}}-\frac{1}{2}}\left(\frac{A_{2}}{y_{2}}\right)^{\frac{\Phi_{2}}{\sigma_{2}^{2}}-\frac{1}{2}} I_{\frac{2 \Phi_{1}}{\sigma_{1}^{2}}-1}\left(2 \sqrt{A_{1} y_{1}}\right) I_{\frac{2 \Phi_{2}}{\sigma_{2}^{2}}-1}\left(2 \sqrt{A_{2} y_{2}}\right) .
\end{gathered}
$$

Simplifying the $\tilde{\mathbf{F}}_{\mathbf{3}}\left(\tau, \eta, \mathbf{s}_{\mathbf{1}}\left(\mathbf{z}_{1}\right), \mathbf{s}_{\mathbf{2}}\left(\mathbf{z}_{2}\right)\right)$ term:

By using similar steps to those presented from (A5.9) - (A5.17) we obtain

$$
\begin{gathered}
\hat{F}_{3}\left(\tau, \eta, v_{1}\left(y_{1}\right), v_{2}\left(y_{2}\right)\right)=-h\left(\tau, \eta, v_{1,0}, v_{2,0}\right) e^{-\left(A_{1}+A_{2}\right)} \frac{2 \Omega_{1}}{\sigma_{1}^{2}\left(e^{\Omega_{1} \tau}-1\right)} \frac{2 \Omega_{2}}{\sigma_{2}^{2}\left(e^{\Omega_{2} \tau}-1\right)} \\
\times \exp \left\{\left[\frac{\left(\Theta_{1}-\Omega_{1}\right)\left(e^{\Omega_{1} \tau}-1\right)}{2 \Omega_{1}}-1\right] y_{1}\right\} \exp \left\{\left[\frac{\left(\Theta_{2}-\Omega_{2}\right)\left(e^{\Omega_{2} \tau}-1\right)}{2 \Omega_{2}}-1\right] y_{2}\right\} \\
\times\left(\frac{A_{1}}{y_{1}}\right)^{\frac{\Phi_{1}}{\sigma_{1}^{2}}-\frac{1}{2}}\left(\frac{A_{2}}{y_{2}}\right)^{\frac{\Phi_{2}}{\sigma_{2}^{2}}-\frac{1}{2}} I_{1-\frac{2 \Phi_{1}}{\sigma_{1}^{2}}}\left(2 \sqrt{A_{1} y_{1}}\right) I_{1-\frac{2 \Phi_{2}}{\sigma_{2}^{2}}}\left(2 \sqrt{A_{2} y_{2}}\right) .
\end{gathered}
$$

\section{Explicit form of the inverse Laplace transform:}

Combining (A5.21), (A5.22) and (A5.23) we conclude that

$$
\hat{U}\left(\tau, \eta, v_{1}\left(y_{1}\right), v_{2}\left(y_{2}\right)\right)=\hat{F}_{1}\left(\tau, \eta, v_{1}\left(y_{1}\right), v_{2}\left(y_{2}\right)\right)+\hat{F}_{2}\left(\tau, \eta, v_{1}\left(y_{1}\right), v_{2}\left(y_{2}\right)\right)+\hat{F}_{3}\left(\tau, \eta, v_{1}\left(y_{1}\right), v_{2}\left(y_{2}\right)\right),
$$

\footnotetext{
${ }^{15}$ We make use of the symmetry relation

$$
I_{1-a}(x)=I_{a-1}(x) .
$$
}


which implies that

$$
\begin{gathered}
\hat{U}\left(\tau, \eta, v_{1}\left(y_{1}\right), v_{2}\left(y_{2}\right)\right)=h\left(\tau, \eta, v_{1,0}, v_{2,0}\right) e^{-\left(A_{1}+y_{1}\right)-\left(A_{2}+y_{2}\right)} \frac{2 \Omega_{1}}{\sigma_{1}^{2}\left(e^{\Omega_{1} \tau}-1\right)} \frac{2 \Omega_{2}}{\sigma_{2}^{2}\left(e^{\Omega_{2} \tau}-1\right)} \\
\times \exp \left\{\left[\frac{\left(\Theta_{1}-\Omega_{1}\right)\left(e^{\Omega_{1} \tau}-1\right)}{2 \Omega_{1}}\right] y_{1}\right\} \exp \left\{\left[\frac{\left(\Theta_{2}-\Omega_{2}\right)\left(e^{\Omega_{2} \tau}-1\right)}{2 \Omega_{2}}\right] y_{2}\right\} \\
\times\left[\left(\frac{A_{1}}{y_{1}}\right)^{\frac{\Phi_{1}}{\sigma_{1}^{2}}-\frac{1}{2}}\left(\frac{A_{2}}{y_{2}}\right)^{\frac{\Phi_{2}}{\sigma_{2}^{2}}-\frac{1}{2}} I_{\frac{2 \Phi_{1}}{\sigma_{1}^{2}}-1}\left(2 \sqrt{A_{1} y_{1}}\right) I_{1-\frac{2 \Phi_{2}}{\sigma_{2}^{2}}}\left(2 \sqrt{A_{2} y_{2}}\right)\right. \\
+\left(\frac{A_{1}}{y_{1}}\right)^{\frac{\Phi_{1}}{\sigma_{1}^{2}}-\frac{1}{2}}\left(\frac{A_{2}}{y_{2}}\right)^{\frac{\Phi_{2}}{\sigma_{2}^{2}}-\frac{1}{2}} I_{1-\frac{2 \Phi_{1}}{\sigma_{1}^{2}}}\left(2 \sqrt{A_{1} y_{1}}\right) I_{\frac{2 \Phi_{2}}{\sigma_{2}^{2}}-1}\left(2 \sqrt{A_{2} y_{2}}\right) \\
\left.-\left(\frac{A_{1}}{y_{1}}\right)^{\frac{\Phi_{1}}{\sigma_{1}^{2}}-\frac{1}{2}}\left(\frac{A_{2}}{y_{2}}\right)^{\frac{\Phi_{2}}{\sigma_{2}^{2}}-\frac{1}{2}} I_{1-\frac{2 \Phi_{1}}{\sigma_{1}^{2}}}\left(2 \sqrt{A_{1} y_{1}}\right) I_{1-\frac{2 \Phi_{2}}{\sigma_{2}^{2}}}\left(2 \sqrt{A_{2} y_{2}}\right)\right] .
\end{gathered}
$$

Now, substituting for $A_{1}, A_{2}, h\left(\tau, \eta, v_{1,0}, v_{2,0}\right), y_{1}$ and $y_{2}$ from equations (A5.1), (A5.3) and (A5.11) we obtain

$$
\begin{aligned}
& \hat{U}\left(\tau, \eta, v_{1}, v_{2}\right)= \exp \left\{\left[\frac{\left(\Phi_{1}-\sigma_{1}^{2}\right)\left(\Theta_{1}-\Omega_{1}\right)}{\sigma_{1}^{2}}+\frac{\left(\Phi_{2}-\sigma_{2}^{2}\right)\left(\Theta_{2}-\Omega_{2}\right)}{\sigma_{2}^{2}}-i \eta(r-q)+\Theta_{1}+\Theta_{2}\right] \tau\right\} \\
& \times \exp \{-\left.\left(\frac{\Theta_{1}-\Omega_{1}}{\sigma_{1}^{2}}\right) v_{1,0}-\left(\frac{\Theta_{2}-\Omega_{2}}{\sigma_{2}^{2}}\right) v_{2,0}+i \eta x_{0}\right\} \frac{2 \Omega_{1}}{\sigma_{1}^{2}\left(e^{\Omega_{1} \tau}-1\right)} \frac{2 \Omega_{2}}{\sigma_{2}^{2}\left(e^{\Omega_{2} \tau}-1\right)} \\
& \times \exp \left\{-\left(\frac{2 \Omega_{1}}{\sigma_{1}^{2}\left(e^{\Omega_{1} \tau}-1\right)}\right)\left(v_{1,0} e^{\Omega_{1} \tau}+v_{1}\right)-\left(\frac{2 \Omega_{2}}{\sigma_{2}^{2}\left(e^{\Omega_{2} \tau}-1\right)}\right)\left(v_{2,0} e^{\Omega_{2} \tau}+v_{2}\right)\right\} \\
& \times \exp \left\{\left(\frac{\Theta_{1}-\Omega_{1}}{\sigma_{1}^{2}}\right) v_{1}\right\} \exp \left\{\left(\frac{\Theta_{2}-\Omega_{2}}{\sigma_{2}^{2}}\right) v_{2}\right\} \\
& \times {\left[\frac{v_{1,0} e^{\Omega_{1} \tau}}{v_{1}}\right)^{\frac{\Phi_{1}}{\sigma_{1}^{2}}-\frac{1}{2}}\left(\frac{v_{2,0} e^{\Omega_{2} \tau}}{v_{2}}\right)^{\frac{\Phi_{2}}{\sigma_{2}^{2}}-\frac{1}{2}} I_{\frac{2 \Phi_{1}}{\sigma_{1}^{2}}-1}\left(\frac{4 \Omega_{1}}{\sigma_{1}^{2}\left(e^{\Omega_{1} \tau}-1\right)}\left(v_{1} v_{1,0} e^{\Omega_{1} \tau}\right)^{\frac{1}{2}}\right) } \\
& \times I_{1-\frac{2 \Phi_{2}}{\sigma_{2}^{2}}}\left(\frac{4 \Omega_{2}}{\sigma_{2}^{2}\left(e^{\Omega_{2} \tau}-1\right)}\left(v_{2} v_{2,0} e^{\Omega_{2} \tau}\right)^{\frac{1}{2}}\right) \\
&+\left(\frac{v_{1,0} e^{\Omega_{1} \tau}}{v_{1}}\right)^{\frac{\Phi_{1}}{\sigma_{1}^{2}}-\frac{1}{2}}\left(\frac{v_{2,0} e^{\Omega_{2} \tau}}{v_{2}}\right)^{\frac{\Phi_{2}}{\sigma_{2}^{2}}-\frac{1}{2}} I_{1-\frac{2 \Phi_{1}}{\sigma_{1}^{2}}}\left(\frac{4 \Omega_{1}}{\sigma_{1}^{2}\left(e^{\Omega_{1} \tau}-1\right)}\left(v_{1} v_{1,0} e^{\Omega_{1} \tau}\right)^{\frac{1}{2}}\right) \\
& \times I_{\frac{2 \Phi_{2}}{\sigma_{2}^{2}}-1}\left(\frac{4 \Omega_{2}}{\sigma_{2}^{2}\left(e^{\Omega_{2} \tau}-1\right)}\left(v_{2} v_{2,0} e^{\Omega_{2} \tau}\right)^{\frac{1}{2}}\right) \\
&-\left(\frac{v_{1,0} e^{\Omega_{1} \tau}}{v_{1}}\right)^{\frac{\Phi_{1}}{\sigma_{1}^{2}}-\frac{1}{2}}\left(\frac{v_{2,0} e^{\Omega_{2} \tau}}{v_{2}}\right)^{\frac{\Phi_{2}}{\sigma_{2}^{2}-\frac{1}{2}}} I_{1-\frac{2 \Phi_{1}}{\sigma_{1}^{2}}}\left(\frac{4 \Omega_{1}}{\sigma_{1}^{2}\left(e^{\Omega_{1} \tau}-1\right)}\left(v_{1} v_{1,0} e^{\Omega_{1} \tau}\right)^{\frac{1}{2}}\right) \\
& \times I_{1-\frac{2 \Phi_{2}}{\sigma_{2}^{2}}}\left(\frac{4 \Omega_{2}}{\sigma_{2}^{2}\left(e^{\Omega_{2} \tau}-1\right)}\left(v_{2} v_{2,0} e^{\Omega_{2} \tau}\right)^{\frac{1}{2}}\right) . \\
&(\mathrm{A} 5.25)
\end{aligned}
$$

After making further simplifications to the above equation and noting again that $I_{\phi-1}(x)=$ 
$I_{1-\phi}(x)$ we obtain

$$
\begin{aligned}
& \hat{U}\left(\tau, \eta, v_{1}, v_{2}\right)=\exp \left\{\left(\frac{\Theta_{1}-\Omega_{1}}{\sigma_{1}^{2}}\right)\left(v_{1}-v_{1,0}+\Phi_{1} \tau\right)+\left(\frac{\Theta_{2}-\Omega_{2}}{\sigma_{2}^{2}}\right)\left(v_{2}-v_{2,0}+\Phi_{2} \tau\right)\right\} \\
& \times \exp \left\{-\left(\frac{2 \Omega_{1}}{\sigma_{1}^{2}\left(e^{\Omega_{1} \tau}-1\right)}\right)\left(v_{1,0} e^{\Omega_{1} \tau}+v_{1}\right)-\left(\frac{2 \Omega_{2}}{\sigma_{2}^{2}\left(e^{\Omega_{2} \tau}-1\right)}\right)\left(v_{2,0} e^{\Omega_{2} \tau}+v_{2}\right)\right\} \\
& \times e^{i \eta x_{0}-i \eta(r-q) \tau} \frac{2 \Omega_{1} e^{\Omega_{1} \tau}}{\sigma_{1}^{2}\left(e^{\Omega_{1} \tau}-1\right)} \frac{2 \Omega_{2} e^{\Omega_{2} \tau}}{\sigma_{2}^{2}\left(e^{\Omega_{2} \tau}-1\right)}\left(\frac{v_{1,0} e^{\Omega_{1} \tau}}{v_{1}}\right)^{\frac{\Phi_{1}}{\sigma_{1}^{2}}-\frac{1}{2}}\left(\frac{v_{2,0} e^{\Omega_{2} \tau}}{v_{2}}\right)^{\frac{\Phi_{2}}{\sigma_{2}^{2}}-\frac{1}{2}} \\
& \times I_{\frac{2 \Phi_{1}}{\sigma_{1}^{2}}-1}\left(\frac{4 \Omega_{1}}{\sigma_{1}^{2}\left(e^{\Omega_{1} \tau}-1\right)}\left(v_{1} v_{1,0} e^{\Omega_{1} \tau}\right)^{\frac{1}{2}}\right) I_{\frac{2 \Phi_{2}}{\sigma_{2}^{2}}-1}\left(\frac{4 \Omega_{2}}{\sigma_{2}^{2}\left(e^{\Omega_{2} \tau}-1\right)}\left(v_{2} v_{2,0} e^{\Omega_{2} \tau}\right)^{\frac{1}{2}}\right),
\end{aligned}
$$

which is equation (8.1). This concludes the proof.

\section{Appendix 6. Proof of Proposition 9.1}

By applying equation (4.2) to equation (8.1), the inverse Fourier Transform of the density function can be represented as

$$
\mathcal{F}^{-1}\left\{\hat{U}\left(\tau, \eta, v_{1}, v_{2}\right)\right\}=\frac{1}{2 \pi} \int_{-\infty}^{\infty} e^{-i \eta x} \hat{U}\left(\tau, \eta, v_{1}, v_{2}\right) d \eta=U\left(\tau, x, v_{1}, v_{2}\right) .
$$

Substituting for $\hat{U}\left(\tau, \eta, v_{1}, v_{2}\right)$ from equation (8.1) we obtain

$$
U\left(\tau, x, v_{1}, v_{2} ; x_{0}, v_{1,0}, v_{2,0}\right)=\frac{1}{2 \pi} \int_{-\infty}^{\infty} e^{i \eta x_{0}} H\left(\tau, x, v_{1}, v_{2} ;-\eta, v_{1,0}, v_{2,0}\right) d \eta
$$

where

$$
\begin{aligned}
& H\left(\tau, x, v_{1}, v_{2} ; \eta, v_{1,0}, v_{2,0}\right)=\exp \left\{\left(\frac{\Theta_{1}-\Omega_{1}}{\sigma_{1}^{2}}\right)\left(v_{1}-v_{1,0}+\Phi_{1} \tau\right)+\left(\frac{\Theta_{2}-\Omega_{2}}{\sigma_{2}^{2}}\right)\left(v_{2}-v_{2,0}+\Phi_{2} \tau\right)\right\} \\
& \times \exp \left\{-\left(\frac{2 \Omega_{1}}{\sigma_{1}^{2}\left(e^{\Omega_{1} \tau}-1\right)}\right)\left(v_{1,0} e^{\Omega_{1} \tau}+v_{1}\right)-\left(\frac{2 \Omega_{2}}{\sigma_{2}^{2}\left(e^{\Omega_{2} \tau}-1\right)}\right)\left(v_{2,0} e^{\Omega_{2} \tau}+v_{2}\right)\right\} \\
& \quad \times e^{i \eta x+i \eta(r-q) \tau} \frac{2 \Omega_{1} e^{\Omega_{1} \tau}}{\sigma_{1}^{2}\left(e^{\Omega_{1} \tau}-1\right)} \frac{2 \Omega_{2} e^{\Omega_{2} \tau}}{\sigma_{2}^{2}\left(e^{\Omega_{2} \tau}-1\right)}\left(\frac{v_{1,0} e^{\Omega_{1} \tau}}{v_{1}}\right)^{\frac{\Phi_{1}}{\sigma_{1}^{2}-\frac{1}{2}}}\left(\frac{v_{2,0} e^{\Omega_{2} \tau}}{v_{2}}\right)^{\frac{\Phi_{2}}{\sigma_{2}^{2}-\frac{1}{2}}} \\
& \quad \times I_{\frac{2 \Phi_{1}}{\sigma_{1}^{2}}-1}\left(\frac{4 \Omega_{1}}{\sigma_{1}^{2}\left(e^{\Omega_{1} \tau}-1\right)}\left(v_{1} v_{1,0} e^{\Omega_{1} \tau}\right)^{\frac{1}{2}}\right) I_{\frac{2 \Phi_{2}}{\sigma_{2}^{2}}-1}\left(\frac{4 \Omega_{2}}{\sigma_{2}^{2}\left(e^{\Omega_{2} \tau}-1\right)}\left(v_{2} v_{2,0} e^{\Omega_{2} \tau}\right)^{\frac{1}{2}}\right) .
\end{aligned}
$$

which is the result given in Proposition 9.1. 


\section{Appendix 7. Some Useful Complex Integrals}

In this appendix we reproduce the integral representation of complex functions given in Adolfsson et al. (2009) and Shephard (1991) as they are required for the calculations in Appendices 8 and 9 . We seek complex integral representations of expressions involving the function $g$ which satisfies the following two conditions:

- $h(\phi) \equiv g(\phi-i)$

- $h(-\phi)=\overline{h(\phi)}$.

We need to consider integrals of the form

$$
Q_{1}=\frac{1}{2 \pi} \int_{-\infty}^{\infty} g(-\phi) \int_{a}^{\infty} e^{y} e^{i \phi y} d y d \phi
$$

and

$$
Q_{2}=\frac{1}{2 \pi} \int_{-\infty}^{\infty} g(-\phi) \int_{a}^{\infty} e^{i \phi y} d y d \phi
$$

Equation (A7.1) is simplified by letting $\xi=\phi-i$. Substituting this into (A7.1) gives

$$
Q_{1}=\frac{1}{2 \pi} \int_{-\infty}^{\infty} g(-\xi-i) \int_{a}^{\infty} e^{i \xi y} d y d \xi
$$

Making a further change of variable $\eta=-\xi$ yields

$$
\begin{aligned}
Q_{1} & =\frac{1}{2 \pi} \int_{-\infty}^{\infty} g(\eta-i) \int_{a}^{\infty} e^{-i \eta y} d y d \eta \\
& =\frac{1}{2 \pi} \int_{-\infty}^{\infty} g(\eta-i)\left[\lim _{b \rightarrow \infty} \frac{e^{-i \eta a}-e^{-i \eta b}}{i \eta}\right] d \eta
\end{aligned}
$$

Equation (A7.4) can be expressed as

$$
\begin{aligned}
Q_{1} & =\frac{1}{2 \pi} \lim _{b \rightarrow \infty}\left[\int_{0}^{\infty} g(\eta-i)\left(\frac{e^{-i \eta a}-e^{-i \eta b}}{i \eta}\right) d \eta+\int_{0}^{\infty} g(-\eta-i)\left(\frac{e^{i \eta a}-e^{i \eta b}}{-i \eta}\right) d \eta\right] \\
& =\frac{1}{2 \pi} \int_{0}^{\infty} \frac{g(\eta-i) e^{-i \eta a}-g(-\eta-i) e^{i \eta a}}{i \eta} d \eta \\
& -\frac{1}{2 \pi} \lim _{b \rightarrow \infty}\left[\int_{0}^{\infty} \frac{g(\eta-i) e^{-i \eta b}-g(-\eta-i) e^{i \eta b}}{i \eta}\right] d \eta .
\end{aligned}
$$

Now using the result in Shephard (1991) that ${ }^{16}$

$$
F(x)=\frac{1}{2}-\frac{1}{2 \pi} \int_{0}^{\infty} \frac{g(\eta-i) e^{-i \eta x}-g(-\eta-i) e^{i \eta x}}{i \eta} d \eta,
$$

\footnotetext{
${ }^{16}$ The function $F$ is defined by $F(x)=\int_{-\infty}^{x} g(\eta) d \eta$.
} 
where $F(x)$ is a cumulative density function we can show that

$$
\begin{aligned}
\lim _{b \rightarrow \infty} \frac{1}{2 \pi} \int_{0}^{\infty} & \frac{g(\eta-i) e^{-i \eta b}-g(-\eta-i) e^{i \eta b}}{i \eta} d \eta \\
& =\lim _{b \rightarrow \infty} \frac{1}{2 \pi} \int_{0}^{\infty} \frac{h(\eta) e^{-i \eta b}-h(-\eta) e^{i \eta b}}{i \eta} d \eta \\
& =\lim _{b \rightarrow \infty}\left[\frac{1}{2}-F(b)\right]=-\frac{1}{2} .
\end{aligned}
$$

Using this result, equation (A7.5) can be represented as

$$
Q_{1}=\frac{1}{2}+\frac{1}{2 \pi} \int_{0}^{\infty} \frac{h(\eta) e^{-i \eta a}-h(-\eta) e^{i \eta a}}{i \eta} d \eta
$$

Also, if $h(\eta)$ and $h(-\eta)$ are complex conjugates ${ }^{17}$ then

$$
\frac{h(-i \eta) e^{i \eta a}}{-i \eta}=\overline{\left(\frac{h(i \eta) e^{-i \eta a}}{i \eta}\right)} .
$$

Using this result (A7.8) simplifies to

$$
Q_{1}=\frac{1}{2}+\frac{1}{\pi} \int_{0}^{\infty} \operatorname{Re}\left(\frac{h(\eta) e^{-i \eta a}}{i \eta}\right) d \eta
$$

Performing similar operations on equation (A7.2) we obtain

$$
Q_{2}=\frac{1}{2}+\frac{1}{\pi} \int_{0}^{\infty} \operatorname{Re}\left(\frac{g(\eta) e^{-i \eta a}}{i \eta}\right) d \eta
$$

\section{Appendix 8. Proof of Proposition 10.1}

By first letting $x=\log (S)$ and making use of the relation $C_{E}\left(\tau, \log (S), v_{1}, v_{2}\right)=V_{E}\left(\tau, S, v_{1}, v_{2}\right)$ which we introduced in equation (3.2), followed by substituting the explicit density function presented in Proposition 9.1 into the European option component in equation (3.15) we obtain after rearranging

$$
\begin{aligned}
V_{E}\left(\tau, S, v_{1}, v_{2}\right)= & e^{-r \tau} \int_{0}^{\infty} \int_{0}^{\infty} \int_{\ln K}^{\infty}\left(e^{x}-K\right)\left[\frac{1}{2 \pi} \int_{-\infty}^{\infty} e^{i \eta x}\right. \\
& \left.\times u_{2}\left(\tau, S, v_{1}, v_{2} ;-\eta, w_{1}, w_{2}\right) d \eta\right] d x d w_{1} d w_{2}
\end{aligned}
$$

\footnotetext{
${ }^{17}$ Note that $h(-\eta)=\overline{h(\eta)}$ represents the complex conjugate.
} 
where

$$
\begin{gathered}
u_{2}\left(\tau, S, v_{1}, v_{2} ; \eta, w_{1}, w_{2}\right)=\exp \left\{\left(\frac{\Theta_{1,2}-\Omega_{1,2}}{\sigma_{1}^{2}}\right)\left(v_{1}-w_{1}+\Phi_{1} \tau\right)+\left(\frac{\Theta_{2,2}-\Omega_{2,2}}{\sigma_{2}^{2}}\right)\left(v_{2}-w_{2}+\Phi_{2} \tau\right)\right\} \\
\times \exp \left\{-\left(\frac{2 \Omega_{1,2}}{\sigma_{1}^{2}\left(e^{\Omega_{1,2} \tau}-1\right)}\right)\left(w_{1} e^{\Omega_{1,2} \tau}+v_{1}\right)-\left(\frac{2 \Omega_{2,2}}{\sigma_{2}^{2}\left(e^{\Omega_{2,2} \tau}-1\right)}\right)\left(w_{2} e^{\Omega_{2,2} \tau}+v_{2}\right)\right\} \\
\times e^{i \eta \ln S+i \eta(r-q) \tau} \frac{2 \Omega_{1,2} e^{\Omega_{1,2} \tau}}{\sigma_{1}^{2}\left(e^{\Omega_{1} \tau}-1\right)} \frac{2 \Omega_{2,2} e^{\Omega_{2,2} \tau}}{\sigma_{2}^{2}\left(e^{\Omega_{2,2} \tau}-1\right)}\left(\frac{w_{1} e^{\Omega_{1,2} \tau}}{v_{1}}\right)^{\frac{\Phi_{1}}{\sigma_{1}^{2}}-\frac{1}{2}}\left(\frac{w_{2} e^{\Omega_{2,2} \tau}}{v_{2}}\right)^{\frac{\Phi_{2}}{\sigma_{2}^{2}}-\frac{1}{2}} \\
\times I_{\frac{2 \Phi_{1}}{\sigma_{1}^{2}}-1}\left(\frac{4 \Omega_{1,2}}{\sigma_{1}^{2}\left(e^{\Omega_{1,2} \tau}-1\right)}\left(v_{1} w_{1} e^{\Omega_{1,2} \tau}\right)^{\frac{1}{2}}\right) I_{\frac{2 \Phi_{2}}{\sigma_{2}^{2}}-1}\left(\frac{4 \Omega_{2,2}}{\sigma_{2}^{2}\left(e^{\Omega_{2,2} \tau}-1\right)}\left(v_{2} w_{2} e^{\Omega_{2,2} \tau}\right)^{\frac{1}{2}}\right),
\end{gathered}
$$

with $\Theta_{1,1}=\Theta_{1}(i-\eta), \Theta_{1,2}=\Theta_{1}(-\eta), \Theta_{2,1}=\Theta_{2}(i-\eta), \Theta_{2,2}=\Theta_{2}(-\eta), \Omega_{1,1}=\Omega_{1}(i-\eta)$, $\Omega_{1,2}=\Omega_{1}(-\eta), \Omega_{2,1}=\Omega_{2}(i-\eta)$ and $\Omega_{2,2}=\Omega_{2}(-\eta)$.

From the above equation, we note that the payoff of the European call option is independent of the running variance variables namely $w_{1}$ and $w_{2}$. This gives us the flexibility to calculate the integrals with respect to $w_{1}$ and $w_{2}$ first thus equation (A8.1) can be written as

$$
\begin{aligned}
& V_{E}\left(\tau, S, v_{1}, v_{2}\right)=\frac{e^{-r \tau}}{2 \pi} \int_{-\infty}^{\infty} \int_{\ln K}^{\infty} e^{i \eta x}\left(e^{x}-K\right) \\
& \times\left[\int_{0}^{\infty} \int_{0}^{\infty} u_{2}\left(\tau, S, v_{1}, v_{2} ;-\eta, w_{1}, w_{2}\right) d w_{1} d w_{2}\right] d \eta d x
\end{aligned}
$$

In evaluating the double integral with respect to the running variance variables, we first let ${ }^{18}$

$$
\left\{\begin{array}{l}
A_{1}=\frac{2 \Omega_{1,2} w_{1}}{\sigma_{1}^{2}\left(1-e^{-\Omega_{1,2} \tau}\right)}, \\
A_{2}=\frac{2 \Omega_{2,2} w_{2}}{\sigma_{2}^{2}\left(1-e^{-\Omega_{2,2} \tau}\right)},
\end{array}\right.
$$

and

$$
\left\{\begin{array}{l}
y_{1}=\frac{2 \Omega_{1,2} v_{1}}{\sigma_{1}^{2}\left(e^{\Omega_{1,2} \tau}-1\right)}, \\
y_{2}=\frac{2 \Omega_{2,2} v_{2}}{\sigma_{2}^{2}\left(e^{\Omega_{2,2} \tau}-1\right)} .
\end{array}\right.
$$

Using $g_{2}\left(\tau, S, v_{1}, v_{2}\right)$ to denote the inner double integral in equation (A8.3) we find that it

\footnotetext{
${ }^{18}$ Note that we have introduced these functions before in the systems (A5.1) and (A5.11).
} 
can be written as

$$
\begin{aligned}
g_{2}\left(\tau, S, v_{1}, v_{2}\right)= & \exp \left\{\left(\frac{\Theta_{1,2}-\Omega_{1,2}}{\sigma_{1}^{2}}\right)\left(v_{1}+\Phi_{1} \tau\right)+\left(\frac{\Theta_{2,2}-\Omega_{2,2}}{\sigma_{2}^{2}}\right)\left(v_{2}+\Phi_{2} \tau\right)\right\} e^{i \eta \ln S+i \eta(r-q) \tau} \\
\times & \exp \left\{-\left(\frac{2 \Omega_{1,2}}{\sigma_{1}^{2}\left(e^{\Omega_{1,2} \tau}-1\right)}\right) v_{1}-\left(\frac{2 \Omega_{2,2}}{\sigma_{2}^{2}\left(e^{\Omega_{2,2} \tau}-1\right)}\right) v_{2}\right\} \\
\times & \int_{0}^{\infty} \int_{0}^{\infty} \exp \left\{-\left[\frac{\left(\Theta_{1,2}-\Omega_{1,2}\right)\left(1-e^{-\Omega_{1,2} \tau}\right)}{2 \Omega_{1,2}}+1\right] A_{1}\right\}\left(\frac{A_{1}}{y_{1}}\right)^{\frac{\Phi_{1}}{\sigma_{1}^{2}}-\frac{1}{2}} \\
& \times \exp \left\{-\left[\frac{\left(\Theta_{2,2}-\Omega_{2,2}\right)\left(1-e^{-\Omega_{2,2} \tau}\right)}{2 \Omega_{2,2}}+1\right] A_{2}\right\}\left(\frac{A_{2}}{y_{2}}\right)^{\frac{\Phi_{2}}{\sigma_{2}^{2}}-\frac{1}{2}} \\
& \times I_{\frac{2 \Phi_{1}}{\sigma_{1}^{2}}-1}\left(2 \sqrt{A_{1} y_{1}}\right) I_{\frac{2 \Phi_{2}}{\sigma_{2}^{2}}-1}\left(2 \sqrt{A_{2} y_{2}}\right) d A_{1} d A_{2},
\end{aligned}
$$

Now using the definition of the modified Bessel function, we can further simplify the above equation to

$$
\begin{aligned}
g_{2}\left(\tau, S, v_{1}, v_{2}\right)= & \exp \left\{\left(\frac{\Theta_{1,2}-\Omega_{1,2}}{\sigma_{1}^{2}}\right)\left(v_{1}+\Phi_{1} \tau\right)+\left(\frac{\Theta_{2,2}-\Omega_{2,2}}{\sigma_{2}^{2}}\right)\left(v_{2}+\Phi_{2} \tau\right)\right\} e^{i \eta \ln S+i \eta(r-q) \tau} \\
\times & \exp \left\{-\left(\frac{2 \Omega_{1,2}}{\sigma_{1}^{2}\left(e^{\Omega_{1,2} \tau}-1\right)}\right) v_{1}-\left(\frac{2 \Omega_{2,2}}{\sigma_{2}^{2}\left(e^{\Omega_{2,2} \tau}-1\right)}\right) v_{2}\right\} \\
\times & \sum_{n_{1}=0}^{\infty} \sum_{n_{2}=0}^{\infty} \frac{y_{1}^{n_{1}}}{n_{1} !} \frac{y_{2}^{n_{2}}}{n_{2} !} \int_{0}^{\infty} \int_{0}^{\infty} \exp \left\{-\left[\frac{\left(\Theta_{1,2}-\Omega_{1,2}\right)\left(1-e^{-\Omega_{1,2} \tau}\right)+2 \Omega_{1,2}}{2 \Omega_{1,2}}\right] A_{1}\right\} \\
\times & \exp \left\{-\left[\frac{\left(\Theta_{2,2}-\Omega_{2,2}\right)\left(1-e^{-\Omega_{2,2} \tau}\right)+2 \Omega_{2,2}}{2 \Omega_{2,2}}\right] A_{2}\right\} \\
& \times \frac{A_{1}^{n_{1}+\frac{2 \Phi_{1}}{\sigma_{1}^{2}}-1}}{\Gamma\left(n_{1}+\frac{2 \Phi_{1}}{\sigma_{1}^{2}}\right)} \frac{A_{2} n_{2}+\frac{2 \Phi_{2}}{\sigma_{2}^{2}}-1}{\Gamma\left(n_{2}+\frac{2 \Phi_{2}}{\sigma_{2}^{2}}\right)} d A_{1} d A_{2} .
\end{aligned}
$$

Let

$$
\xi_{1}=\left[\frac{\left(\Theta_{1,2}-\Omega_{1,2}\right)\left(1-e^{-\Omega_{1,2} \tau}\right)+2 \Omega_{1,2}}{2 \Omega_{1,2}}\right] A_{1}, \quad \xi_{2}=\left[\frac{\left(\Theta_{2,2}-\Omega_{2,2}\right)\left(1-e^{-\Omega_{2,2} \tau}\right)+2 \Omega_{2,2}}{2 \Omega_{2,2}}\right] A_{2} .
$$

Substituting these into (A8.7) we obtain

$$
\begin{aligned}
& g_{2}\left(\tau, S, v_{1}, v_{2}\right)=\exp \left\{\left(\frac{\Theta_{1,2}-\Omega_{1,2}}{\sigma_{1}^{2}}\right)\left(v_{1}+\Phi_{1} \tau\right)+\left(\frac{\Theta_{2,2}-\Omega_{2,2}}{\sigma_{2}^{2}}\right)\left(v_{2}+\Phi_{2} \tau\right)\right\} e^{i \eta \ln S+i \eta(r-q) \tau} \\
& \quad \times \exp \left\{-\left(\frac{2 \Omega_{1,2}}{\sigma_{1}^{2}\left(e^{\Omega_{1,2} \tau}-1\right)}\right) v_{1}-\left(\frac{2 \Omega_{2,2}}{\sigma_{2}^{2}\left(e^{\Omega_{2,2} \tau}-1\right)}\right) v_{2}\right\} \\
& \quad \times \sum_{n_{1}=0}^{\infty} \sum_{n_{2}=0}^{\infty} \frac{y_{1}^{n_{1}}}{n_{1} ! \Gamma\left(n_{1}+\frac{2 \Phi_{1}}{\sigma_{1}^{2}}\right)} \frac{y_{2}^{n_{2}}}{n_{2} ! \Gamma\left(n_{2}+\frac{2 \Phi_{2}}{\sigma_{2}^{2}}\right)}\left(\frac{2 \Omega_{1,2}}{\left(\Theta_{1,2}-\Omega_{1,2}\right)\left(1-e^{-\Omega_{1,2} \tau}\right)+2 \Omega_{1,2}}\right)^{n_{1}+\frac{2 \Phi_{1}}{\sigma_{1}^{2}}} \\
& \quad \times\left(\frac{2 \Omega_{2,2}}{\left(\Theta_{2,2}-\Omega_{2,2}\right)\left(1-e^{-\Omega_{2,2} \tau}\right)+2 \Omega_{2,2}}\right)^{n_{2}+\frac{2 \Phi_{2}}{\sigma_{2}^{2}}} \int_{0}^{\infty} \int_{0}^{\infty} e^{-\xi_{1}-\xi_{2}} \xi_{1}^{n_{1}+\frac{2 \Phi_{1}}{\sigma_{1}^{2}}-1} \xi_{2}^{n_{2}+\frac{2 \Phi_{2}}{\sigma_{2}^{2}}-1} d \xi_{1} d \xi_{2} .
\end{aligned}
$$

By noting that

$$
\int_{0}^{\infty} \int_{0}^{\infty} e^{-\xi_{1}-\xi_{2}} \xi_{1}^{n_{1}+\frac{2 \Phi_{1}}{\sigma_{1}^{2}}-1} \xi_{2}^{n_{2}+\frac{2 \Phi_{2}}{\sigma_{2}^{2}}-1} d \xi_{1} d \xi_{2}=\Gamma\left(n_{1}+\frac{2 \Phi_{1}}{\sigma_{1}^{2}}\right) \Gamma\left(n_{2}+\frac{2 \Phi_{2}}{\sigma_{2}^{2}}\right),
$$


we obtain

$$
\begin{aligned}
& g_{2}\left(\tau, S, v_{1}, v_{2}\right)=\exp \left\{\left(\frac{\Theta_{1,2}-\Omega_{1,2}}{\sigma_{1}^{2}}\right)\left(v_{1}+\Phi_{1} \tau\right)+\left(\frac{\Theta_{2,2}-\Omega_{2,2}}{\sigma_{2}^{2}}\right)\left(v_{2}+\Phi_{2} \tau\right)\right\} e^{i \eta \ln S+i \eta(r-q) \tau} \\
& \quad \times \exp \left\{-\left(\frac{2 \Omega_{1,2}}{\sigma_{1}^{2}\left(e^{\Omega_{1,2} \tau}-1\right)}\right) v_{1}-\left(\frac{2 \Omega_{2,2}}{\sigma_{2}^{2}\left(e^{\Omega_{2,2} \tau}-1\right)}\right) v_{2}\right\} \\
& \quad \times \sum_{n_{1}=0}^{\infty} \sum_{n_{2}=0}^{\infty} \frac{y_{1}^{n_{1}}}{n_{1} !} \frac{y_{2}^{n_{2}}}{n_{2} !}\left(\frac{2 \Omega_{1,2}}{\left(\Theta_{1,2}-\Omega_{1,2}\right)\left(1-e^{-\Omega_{1,2} \tau}\right)+2 \Omega_{1,2}}\right)^{n_{1}+\frac{2 \Phi_{1}}{\sigma_{1}^{2}}} \\
& \quad \times\left(\frac{2 \Omega_{2,2}}{\left(\Theta_{2,2}-\Omega_{2,2}\right)\left(1-e^{-\Omega_{2,2} \tau}\right)+2 \Omega_{2,2}}\right)^{n_{2}+\frac{2 \Phi_{2}}{\sigma_{2}^{2}}} \frac{\Gamma\left(n_{1}+\frac{2 \Phi_{1}}{\sigma_{1}^{2}}\right)}{\Gamma\left(n_{1}+\frac{2 \Phi_{1}}{\sigma_{1}^{2}}\right)} \frac{\Gamma\left(n_{2}+\frac{2 \Phi_{2}}{\sigma_{2}^{2}}\right)}{\Gamma\left(n_{2}+\frac{2 \Phi_{2}}{\sigma_{2}^{2}}\right)} .
\end{aligned}
$$

The above equation simplifies to

$$
\begin{aligned}
& g_{2}\left(\tau, S, v_{1}, v_{2}\right)= \exp \left\{\left(\frac{\Theta_{1,2}-\Omega_{1,2}}{\sigma_{1}^{2}}\right)\left(v_{1}+\Phi_{1} \tau\right)+\left(\frac{\Theta_{2,2}-\Omega_{2,2}}{\sigma_{2}^{2}}\right)\left(v_{2}+\Phi_{2} \tau\right)\right\} e^{i \eta \ln S+i \eta(r-q) \tau} \\
& \times \exp \left\{-\left(\frac{2 \Omega_{1,2}}{\sigma_{1}^{2}\left(e^{\Omega_{1,2} \tau}-1\right)}\right) v_{1}-\left(\frac{2 \Omega_{2,2}}{\sigma_{2}^{2}\left(e^{\Omega_{2,2} \tau}-1\right)}\right) v_{2}\right\} \\
& \times\left(\frac{2 \Omega_{1,2}}{\left(\Theta_{1,2}-\Omega_{1,2}\right)\left(1-e^{-\Omega_{1,2} \tau}\right)+2 \Omega_{1,2}}\right)^{\frac{2 \Phi_{1}}{\sigma_{1}^{2}}}\left(\frac{2 \Omega_{2,2}}{\left(\Theta_{2,2}-\Omega_{2,2}\right)\left(1-e^{-\Omega_{2,2} \tau}\right)+2 \Omega_{2,2}}\right)^{\frac{2 \Phi_{2}}{\sigma_{2}^{2}}} \\
& \quad \times \sum_{n_{1}=0}^{\infty} \sum_{n_{2}=0}^{\infty} \frac{y_{1}^{n_{1}}}{n_{1} !} \frac{y_{2}^{n_{2}}}{n_{2} !}\left(\frac{2 \Omega_{1,2}}{\left(\Theta_{1,2}-\Omega_{1,2}\right)\left(1-e^{-\Omega_{1,2} \tau}\right)+2 \Omega_{1,2}}\right)^{n_{1}} \\
& \quad \times\left(\frac{2 \Omega_{2,2}}{\left(\Theta_{2,2}-\Omega_{2,2}\right)\left(1-e^{-\Omega_{2,2} \tau}\right)+2 \Omega_{2,2}}\right)^{n_{2}} .
\end{aligned}
$$

Now applying Taylor series expansion of the exponential function to the double summation we obtain

$$
\begin{aligned}
& g_{2}\left(\tau, S, v_{1}, v_{2}\right)=\exp \left\{\left(\frac{\Theta_{1,2}-\Omega_{1,2}}{\sigma_{1}^{2}}\right)\left(v_{1}+\Phi_{1} \tau\right)+\left(\frac{\Theta_{2,2}-\Omega_{2,2}}{\sigma_{2}^{2}}\right)\left(v_{2}+\Phi_{2} \tau\right)\right\} e^{i \eta \ln S+i \eta(r-q) \tau} \\
& \quad \times \exp \left\{-\left(\frac{2 \Omega_{1,2}}{\sigma_{1}^{2}\left(e^{\Omega_{1,2} \tau}-1\right)}\right) v_{1}-\left(\frac{2 \Omega_{2,2}}{\sigma_{2}^{2}\left(e^{\Omega_{2,2} \tau}-1\right)}\right) v_{2}\right\} \\
& \quad \times\left(\frac{2 \Omega_{1,2}}{\left(\Theta_{1,2}-\Omega_{1,2}\right)\left(1-e^{-\Omega_{1,2} \tau}\right)+2 \Omega_{1,2}}\right)^{\frac{2 \Phi_{1}}{\sigma_{1}^{2}}}\left(\frac{2 \Omega_{2,2}}{\left(\Theta_{2,2}-\Omega_{2,2}\right)\left(1-e^{-\Omega_{2,2} \tau}\right)+2 \Omega_{2,2}}\right)^{\frac{2 \Phi_{2}}{\sigma_{2}^{2}}} \\
& \quad \times \exp \left\{\frac{2 \Omega_{1,2} y_{1}}{\left(\Theta_{1,2}-\Omega_{1,2}\right)\left(1-e^{-\Omega_{1,2} \tau}\right)+2 \Omega_{1,2}}+\frac{2 \Omega_{2}}{\left(\Theta_{2,2}-\Omega_{2,2}\right)\left(1-e^{-\Omega_{2,2} \tau}\right)+2 \Omega_{2,2}}\right\} .
\end{aligned}
$$

Reverting to the $v_{1}$ and $v_{2}$ variables from the system (A8.5) we obtain

$$
\begin{aligned}
g_{2}(\tau, & \left.S, v_{1}, v_{2}\right)=\exp \left\{\left(\frac{\Theta_{1,2}-\Omega_{1,2}}{\sigma_{1}^{2}}\right)\left(v_{1}+\Phi_{1} \tau\right)+\left(\frac{\Theta_{2,2}-\Omega_{2,2}}{\sigma_{2}^{2}}\right)\left(v_{2}+\Phi_{2} \tau\right)\right\} e^{i \eta \ln S+i \eta(r-q) \tau} \\
& \times \exp \left\{-\left(\frac{2 \Omega_{1,2}}{\sigma_{1}^{2}\left(e^{\Omega_{1,2} \tau}-1\right)}\right) v_{1}-\left(\frac{2 \Omega_{2,2}}{\sigma_{2}^{2}\left(e^{\Omega_{2,2} \tau}-1\right)}\right) v_{2}\right\} \\
& \left.\times\left(\frac{2 \Omega_{1,2}}{\left(\Theta_{1,2}-\Omega_{1,2}\right)\left(1-e^{-\Omega_{1,2} \tau}\right)+2 \Omega_{1,2}}\right)^{\frac{2 \Phi_{1}}{\sigma_{1}^{2}}}\left(\frac{2 \Omega_{2,2}}{\left(\Theta_{2,2}-\Omega_{2,2}\right)\left(1-e^{-\Omega_{2,2} \tau}\right)+2 \Omega_{2,2}}\right)^{\frac{2 \Phi_{2}}{\sigma_{2}^{2}}} \times \frac{2 \Omega_{1,2} v_{1}}{\sigma_{1}^{2}\left(e^{\Omega_{1,2} \tau}-1\right)}\right\} \\
& \times \exp \left\{\frac{2 \Omega_{1,2}}{\left(\Theta_{1,2}-\Omega_{1,2}\right)\left(1-e^{-\Omega_{1,2} \tau}\right)+2 \Omega_{1,2}} \times \frac{2 \Omega_{2,2} v_{2}}{\sigma_{2}^{2}\left(e^{\Omega_{2,2} \tau}-1\right)}\right\} . \\
& \times \exp \left\{\frac{2 \Omega_{2,2}}{\left(\Theta_{2,2}-\Omega_{2,2}\right)\left(1-e^{-\Omega_{2,2} \tau}\right)+2 \Omega_{2,2}}\right.
\end{aligned}
$$


For convenience, we now attempt to represent this density in the form presented in Heston (1993). This is accomplished by adopting the representation

$$
g_{2}\left(\tau, S, v_{1}, v_{2} ;-\eta\right)=\exp \left(-i \eta \ln S+B_{2}(\tau,-\eta)+D_{1,2}(\tau,-\eta) v_{1}+D_{2,2}(\tau,-\eta) v_{2}\right),
$$

where

$$
\begin{aligned}
B_{2}(\tau, \eta)= & i \eta(r-q) \tau+\frac{\Phi_{1}}{\sigma_{1}^{2}}\left\{\left(\Theta_{1,2}-\Omega_{1,2}\right) \tau-2 \ln \left(\frac{\left(\Theta_{1,2}-\Omega_{1,2}\right)\left(1-e^{\Omega_{1,2} \tau}\right)+2 \Omega_{1,2}}{2 \Omega_{1,2}}\right)\right\} \\
& +\frac{\Phi_{2}}{\sigma_{2}^{2}}\left\{\left(\Theta_{2,2}-\Omega_{2,2}\right) \tau-2 \ln \left(\frac{\left(\Theta_{2,2}-\Omega_{2,2}\right)\left(1-e^{\Omega_{2,2} \tau}\right)+2 \Omega_{2,2}}{2 \Omega_{2,2}}\right)\right\}, \\
D_{1,2}(\tau, \eta)= & \frac{\left(\Theta_{1,2}-\Omega_{1,2}\right)}{\sigma_{1}^{2}}-\frac{2 \Omega_{1,2}}{\sigma_{1}^{2}\left(e^{\Omega_{1,2} \tau}-1\right)}+\frac{2 \Omega_{1,2}}{\left(\Theta_{1,2}-\Omega_{1,2}\right)\left(1-e^{-\Omega_{1,2} \tau}\right)+2 \Omega_{1,2}} \times \frac{2 \Omega_{1,2} v_{1}}{\sigma_{1}^{2}\left(e^{\Omega_{1,2} \tau}-1\right)}, \\
D_{2,2}(\tau, \eta)= & \frac{\left(\Theta_{2,2}-\Omega_{2,2}\right)}{\sigma_{2}^{2}}-\frac{2 \Omega_{2,2}}{\sigma_{2}^{2}\left(e^{\Omega_{2,2} \tau}-1\right)}+\frac{2 \Omega_{2,2} v_{2}}{\left(\Theta_{2,2}-\Omega_{2,2}\right)\left(1-e^{-\Omega_{2,2} \tau}\right)+2 \Omega_{2,2}} \times \frac{2 e_{2}^{2}\left(e^{\Omega_{2,2} \tau}-1\right)}{\sigma_{2}}
\end{aligned}
$$

By letting

$$
Q_{1,2}=\frac{\Theta_{1,2}+\Omega_{1,2}}{\Theta_{1,2}-\Omega_{1,2}} \quad \text { and } \quad Q_{2,2}=\frac{\Theta_{2,2}+\Omega_{2,2}}{\Theta_{2,2}-\Omega_{2,2}}
$$

the above three functions reduce to

$$
\begin{aligned}
B_{2}(\tau, \eta)= & i \eta(r-q) \tau+\frac{\Phi_{1}}{\sigma_{1}^{2}}\left\{\left(\Theta_{1,2}+\Omega_{1,2}\right) \tau-2 \ln \left(\frac{1-Q_{1,2} e^{\Omega_{1,2} \tau}}{1-Q_{1,2}}\right)\right\} \\
& +\frac{\Phi_{2}}{\sigma_{2}^{2}}\left\{\left(\Theta_{2,2}+\Omega_{2,2}\right) \tau-2 \ln \left(\frac{1-Q_{2,2} e^{\Omega_{2,2} \tau}}{1-Q_{2,2}}\right)\right\} \\
D_{1,2}(\tau, \eta)= & \frac{\left(\Theta_{1,2}+\Omega_{1,2}\right)}{\sigma_{1}^{2}}\left[\frac{1-e^{\Omega_{1,2} \tau}}{1-Q_{1,2} e^{\Omega_{1,2} \tau}}\right] \\
D_{2,2}(\tau, \eta)= & \frac{\left(\Theta_{2,2}+\Omega_{2,2}\right)}{\sigma_{2}^{2}}\left[\frac{1-e^{\Omega_{2,2} \tau}}{1-Q_{2,2} e^{\Omega_{2,2} \tau}}\right] .
\end{aligned}
$$

Substituting equation (A8.14) into equation (A8.3) we obtain

$$
\begin{array}{r}
V_{E}\left(\tau, S, v_{1}, v_{2}\right)=\frac{e^{-r \tau}}{2 \pi} \int_{-\infty}^{\infty} g_{2}\left(\tau, S, v_{1}, v_{2} ;-\eta\right) \int_{\ln K}^{\infty} e^{x} e^{i \eta x} d x d \eta \\
-K \int_{-\infty}^{\infty} g_{2}\left(\tau, S, v_{1}, v_{2} ;-\eta\right) \int_{\ln K}^{\infty} e^{i \eta x} d x d \eta
\end{array}
$$

The two components on the RHS of the above equation have similar properties to equations (A7.1) and (A7.2) respectively described in Appendix 7. We can evaluate the integrals in equation (A8.18) using equations (A7.10) and (A7.11) provided that $g_{2}\left(\tau, S, v_{1}, v_{2} ; \eta-i\right)$ satisfies appropriate assumptions. The first assumption we we must verify is that $g_{2}\left(\tau, S, v_{1}, v_{2} ; \eta-\right.$ i) can be expressed as a function of $\eta$. This assumption is satisfied since

$$
g_{2}\left(\tau, S, v_{1}, v_{2} ; \eta-i\right)=S e^{(r-q) \tau} g_{1}\left(\tau, S, v_{1}, v_{2} ; \eta\right)
$$


where

$$
g_{1}\left(\tau, S, v_{1}, v_{2} ; \eta\right)=\exp \left(i \eta \ln S+B_{2}(\tau, \eta)+D_{1,1}(\tau, \eta) v_{1}+D_{2,1}(\tau,-\eta) v_{2}\right)
$$

with

$$
\begin{aligned}
B_{1}(\tau, \eta)= & i \eta(r-q) \tau+\frac{\Phi_{1}}{\sigma_{1}^{2}}\left\{\left(\Theta_{1,1}+\Omega_{1,1}\right) \tau-2 \ln \left(\frac{1-Q_{1,2} e^{\Omega_{1,1} \tau}}{1-Q_{1,1}}\right)\right\} \\
& +\frac{\Phi_{2}}{\sigma_{2}^{2}}\left\{\left(\Theta_{2,1}+\Omega_{2,1}\right) \tau-2 \ln \left(\frac{1-Q_{2,1} e^{\Omega_{2,1} \tau}}{1-Q_{2,1}}\right)\right\}, \\
D_{1,1}(\tau, \eta)= & \frac{\left(\Theta_{1,1}+\Omega_{1,1}\right)}{\sigma_{1}^{2}}\left[\frac{1-e^{\Omega_{1,1} \tau}}{1-Q_{1,1} e^{\Omega_{1,1} \tau}}\right], \\
D_{2,1}(\tau, \eta)= & \frac{\left(\Theta_{2,1}+\Omega_{2,1}\right)}{\sigma_{2}^{2}}\left[\frac{1-e^{\Omega_{2,1} \tau}}{1-Q_{2,1} e^{\Omega_{2,1} \tau}}\right] .
\end{aligned}
$$

Furthermore, by using the same reasoning as in equation (A7.9) it can also be shown that

$$
g_{j}\left(\tau, S, v_{1}, v_{2} ;-\eta\right)=\overline{g_{j}\left(\tau, S, v_{1}, v_{2} ; \eta,\right)}, \quad \text { for } \quad j=1,2
$$

and hence all the assumptions required to carry out the calculations yielding (A7.10) and (A7.11) are satisfied. Thus equation (A8.18) becomes

$$
V_{E}\left(\tau, S, v_{1}, v_{2}\right)=e^{-q \tau} S P_{1}\left(\tau, S, v_{1}, v_{2} ; K\right)-e^{-r \tau} K P_{2}\left(\tau, S, v_{1}, v_{2} ; K\right)
$$

where

$$
P_{j}\left(\tau, S, v_{1}, v_{2} ; K\right)=\frac{1}{2}+\frac{1}{\pi} \int_{0}^{\infty} \operatorname{Re}\left(\frac{g_{j}\left(\tau, S, v_{1}, v_{2} ; \eta\right) e^{-i \eta \ln K}}{i \eta}\right) d \eta
$$

for $j=1,2$ which is the result in Proposition 10.1 .

\section{Appendix 9. Proof of Proposition 10.2}

We proceed as we did in Appendix 8 by first letting $x=\log (S)$ and making use of the relation

$C_{P}\left(\tau, \log (S), v_{1}, v_{2}\right)=V_{P}\left(\tau, S, v_{1}, v_{2}\right)$ introduced in equation (3.2). Substituting the density function presented in Proposition 9.1 to the early exercise premium component in equation (3.16) we obtain after rearranging

$$
\begin{aligned}
V_{P}\left(\tau, S, v_{1}, v_{2}\right) & =\int_{0}^{\tau} e^{-r(\tau-\xi)} \int_{0}^{\infty} \int_{0}^{\infty} \int_{\ln b\left(\xi, w_{1}, w_{2}\right)}^{\infty}\left[q e^{y}-r K\right] \\
& \times\left[\frac{1}{2 \pi} \int_{-\infty}^{\infty} e^{i \eta y} g_{2}^{A}\left(\tau-\xi, S, v_{1}, v_{2} ;-\eta, w_{1}, w_{2}\right) d \eta\right] d y d w_{1} d w_{2} d \xi
\end{aligned}
$$


where

$$
\begin{aligned}
& g_{2}^{A}\left(\tau-\xi, S, v_{1}, v_{2} ; \eta, w_{1}, w_{2}\right)=\exp \left\{\left(\frac{\Theta_{1,2}-\Omega_{1,2}}{\sigma_{1}^{2}}\right)\left(v_{1}-w_{1}+\Phi_{1}(\tau-\xi)\right)\right. \\
& \left.\quad+\left(\frac{\Theta_{2,2}-\Omega_{2,2}}{\sigma_{2}^{2}}\right)\left(v_{2}-w_{2}+\Phi_{2}(\tau-\xi)\right)\right\} \\
& \times \exp \left\{-\left(\frac{2 \Omega_{1,2}}{\sigma_{1}^{2}\left(e^{\Omega_{1,2}(\tau-\xi)}-1\right)}\right)\left(w_{1} e^{\Omega_{1,2}(\tau-\xi)}+v_{1}\right)-\left(\frac{2 \Omega_{2,2}}{\sigma_{2}^{2}\left(e^{\Omega_{2,2}(\tau-\xi)}-1\right)}\right)\left(w_{2} e^{\Omega_{2,2}(\tau-\xi)}+v_{2}\right)\right\} \\
& \quad \times e^{i \eta \ln S+i \eta(r-q)(\tau-\xi)} \frac{2 \Omega_{1,2} e^{\Omega_{1,2}(\tau-\xi)}}{\sigma_{1}^{2}\left(e^{\Omega_{1}(\tau-\xi)}-1\right)} \frac{2 \Omega_{2,2} e^{\Omega_{2,2}(\tau-\xi)}}{\sigma_{2}^{2}\left(e^{\Omega_{2,2}(\tau-\xi)}-1\right)}\left(\frac{w_{1} e^{\Omega_{1,2}(\tau-\xi)}}{v_{1}}\right)^{\frac{\Phi_{1}}{\sigma_{1}^{2}}-\frac{1}{2}}\left(\frac{w_{2} e^{\Omega_{2,2}(\tau-\xi)}}{v_{2}}\right)^{\frac{\Phi_{2}}{\sigma_{2}^{2}}-\frac{1}{2}} \\
& \quad \times I_{\frac{2 \Phi_{1}}{\sigma_{1}^{2}}-1}\left(\frac{4 \Omega_{1,2}}{\sigma_{1}^{2}\left(e^{\Omega_{1,2}(\tau-\xi)}-1\right)}\left(v_{1} w_{1} e^{\Omega_{1,2}(\tau-\xi)}\right)^{\frac{1}{2}}\right) I_{\frac{2 \Phi_{2}}{\sigma_{2}^{2}}-1}\left(\frac{4 \Omega_{2,2}}{\sigma_{2}^{2}\left(e^{\Omega_{2,2}(\tau-\xi)}-1\right)}\left(v_{2} w_{2} e^{\Omega_{2,2}(\tau-\xi)}\right)^{\frac{1}{2}}\right) .
\end{aligned}
$$

Equation (A9.1) is equivalent to

$$
\begin{gathered}
V_{P}\left(\tau, S, v_{1}, v_{2}\right)=\int_{0}^{\tau} e^{-r(\tau-\xi)} \int_{0}^{\infty} \int_{0}^{\infty} \frac{1}{2 \pi}\left[q \int_{-\infty}^{\infty} g_{2}^{A}\left(\tau-\xi, S, v_{1}, v_{2} ;-\eta, w_{1}, w_{2}\right) \int_{\ln b\left(\xi, w_{1}, w_{2}\right)}^{\infty} e^{y} e^{i \eta y} d y d \eta\right. \\
\left.-r K \int_{-\infty}^{\infty} g_{2}^{A}\left(\tau-\xi, S, v_{1}, v_{2} ;-\eta, w_{1}, w_{2}\right) \int_{\ln b\left(\xi, w_{1}, w_{2}\right)}^{\infty} e^{i \eta y} d y d \eta\right] d w_{1} d w_{2} d \xi .
\end{gathered}
$$

By proceeding in the same way that we handled equation (A8.18) when simplifying the complex integrals we can write

$$
g_{2}^{A}\left(\tau-\xi, S, v_{1}, v_{2} ; \eta-i, w_{1}, w_{2}\right)=e^{(r-q)(\tau-\xi)} S g_{1}^{A}\left(\tau-\xi, S, v_{1}, v_{2} ; \eta, w_{1}, w_{2}\right)
$$

so that equation (A9.3) reduces to

$$
\begin{gathered}
V_{P}\left(\tau, S, v_{1}, v_{2}\right)=\int_{0}^{\tau} \int_{0}^{\infty} \int_{0}^{\infty}\left[q e^{-q(\tau-\xi)} S P_{1}^{A}\left[\tau-\xi, S, v_{1}, v_{2} ; w_{1}, w_{2}, b\left(\xi, w_{1}, w_{2}\right)\right]\right. \\
\left.-r e^{-r(\tau-\xi)} K P_{2}^{A}\left[\tau-\xi, S, v_{1}, v_{2} ; w_{1}, w_{2}, b\left(\xi, w_{1}, w_{2}\right)\right]\right] d w_{1} d w_{2} d \xi
\end{gathered}
$$

where

$P_{j}^{A}\left(\tau-\xi, S, v_{1}, v_{2} ; w_{1}, w_{2}, b\left(\xi, w_{1}, w_{2}\right)\right)=\frac{1}{2}+\frac{1}{\pi} \int_{0}^{\infty} \operatorname{Re}\left(\frac{g_{j}^{A}\left(\tau-\xi, S, v_{1}, v_{2} ; \eta, w_{1}, w_{2}\right) e^{-i \eta \ln b\left(\xi, w_{1}, w_{2}\right)}}{i \eta}\right) d \eta$

for $j=1,2$ which is the result given in Proposition 10.2 .

\section{Appendix 10. Proof of Proposition 11.1}

Going back to equation (A9.1), we have expressed the early exercise premium value as

$$
\begin{gathered}
V_{P}^{A}\left(\tau, S, v_{1}, v_{2}\right)=\int_{0}^{\tau} e^{-r(\tau-\xi)} \int_{0}^{\infty} \int_{0}^{\infty} \frac{1}{2 \pi}\left[q \int_{-\infty}^{\infty} g_{2}^{A}\left(\tau-\xi, S, v_{1}, v_{2} ;-\eta, w_{1}, w_{2}\right)\right. \\
\times \int_{\ln b\left(\xi, w_{1}, w_{2}\right)}^{\infty} e^{y} e^{i \eta y} d y d \eta \\
\left.-r K \int_{-\infty}^{\infty} g_{2}^{A}\left(\tau-\xi, S, v_{1}, v_{2} ;-\eta, w_{1}, w_{2}\right) \int_{\ln b\left(\xi, w_{1}, w_{2}\right)}^{\infty} e^{i \eta y} d y d \eta\right] d w_{1} d w_{2} d \xi
\end{gathered}
$$


With the approximation, $\ln b\left(\tau, v_{1}, v_{2}\right)=b_{0}(\tau)+b_{1}(\tau) v_{1}+b_{2}(\tau) v_{2}$, the above equation is transformed to

$$
\begin{aligned}
& V_{P}^{A}\left(\tau, S, v_{1}, v_{2}\right)=\int_{0}^{\tau} e^{-r(\tau-\xi)} \int_{0}^{\infty} \int_{0}^{\infty} \frac{1}{2 \pi}\left[q \int_{-\infty}^{\infty} g_{2}^{A}\left(\tau-\xi, S, v_{1}, v_{2} ;-\eta, w_{1}, w_{2}\right)\right. \\
& \quad \times \int_{b_{0}(\xi)+b_{1}(\xi) w_{1}+b_{2}(\xi) w_{2}}^{\infty} e^{y} e^{i \eta y} d y d \eta \\
& \left.\quad-r K \int_{-\infty}^{\infty} g_{2}^{A}\left(\tau-\xi, S, v_{1}, v_{2} ;-\eta, w_{1}, w_{2}\right) \int_{b_{0}(\xi)+b_{1}(\xi) w_{1}+b_{2}(\xi) w_{2}}^{\infty} e^{i \eta y} d y d \eta\right] d w_{1} d w_{2} d \xi
\end{aligned}
$$

By letting $z=y-b_{1}(\xi) w_{1}-b_{2}(\xi) w_{2}$ and substituting this into equation (A10.1) we obtain

$$
\begin{aligned}
& V_{P}^{A}\left(\tau, S, v_{1}, v_{2}\right)=\int_{0}^{\tau} e^{-r(\tau-\xi)} \int_{0}^{\infty} \int_{0}^{\infty} \frac{1}{2 \pi}\left[q \int_{-\infty}^{\infty} g_{2}^{A}\left(\tau-\xi, S, v_{1}, v_{2} ;-\eta, w_{1}, w_{2}\right)\right. \\
& \times \int_{b_{0}(\xi)}^{\infty} e^{(1+i \eta)\left(z+b_{1}(\xi) w_{1}+b_{2}(\xi) w_{2}\right)} d z d \eta \\
& \left.-r K \int_{-\infty}^{\infty} g_{2}^{A}\left(\tau-\xi, S, v_{1}, v_{2} ;-\eta, w_{1}, w_{2}\right) \int_{b_{0}(\xi)}^{\infty} e^{i \eta\left(z+b_{1}(\xi) w_{1}+b_{2}(\xi) w_{2}\right)} d z d \eta\right] d w_{1} d w_{2} d \xi
\end{aligned}
$$

This can be further simplified to

$$
V_{P}^{A}\left(\tau, S, v_{1}, v_{2}\right)=\int_{0}^{\tau} e^{-r(\tau-\xi)} \int_{b_{0}(\xi)}^{\infty}\left[q e^{z} J_{1}(\xi, z)-r K J_{2}(\xi, z)\right] d z d \xi,
$$

where

$$
\begin{array}{r}
J_{1}(\xi, z)=\int_{0}^{\infty} \int_{0}^{\infty} e^{b_{1}(\xi) w_{1}+b_{2}(\xi) w_{2}+i \eta\left(z+b_{1}(\xi) w_{1}+b_{2}(\xi) w_{2}\right)} \\
\times \frac{1}{2 \pi} \int_{-\infty}^{\infty} g_{2}^{A}\left(\tau-\xi, S, v_{1}, v_{2} ;-\eta, w_{1}, w_{2}\right) d \eta d w_{1} d w_{2}
\end{array}
$$

and

$$
J_{2}(\xi, z)=\int_{0}^{\infty} \int_{0}^{\infty} e^{i \eta\left[b_{1}(\xi) w_{1}+b_{2}(\xi) w_{2}\right]} \frac{1}{2 \pi} \int_{-\infty}^{\infty} e^{i \eta z} g_{2}^{A}\left(\tau-\xi, S, v_{1}, v_{2} ;-\eta, w_{1}, w_{2}\right) d \eta d w_{1} d w_{2} .
$$

The two expressions for $J_{1}(\xi, z)$ and $J_{2}(\xi, z)$ are now in a form that can allow us to simplify the integrals with respect to $w_{1}$ and $w_{2}$. By making use of equations (A8.4) and (A8.5) followed by repeating similar steps to those from equations (A8.6)-(A8.13) in Appendix 8 we 
note that $J_{2}(\xi, z)$ becomes

$$
\begin{aligned}
J_{2}(\xi, z) & =\frac{1}{2 \pi} \int_{-\infty}^{\infty}\left[\exp \left\{\left(\frac{\Theta_{1,2}-\Omega_{1,2}}{\sigma_{1}^{2}}\right)\left(v_{1}+\Phi_{1}(\tau-\xi)\right)+\left(\frac{\Theta_{2,2}-\Omega_{2,2}}{\sigma_{2}^{2}}\right)\left(v_{2}+\Phi_{2}(\tau-\xi)\right)\right\}\right. \\
\times & \exp \left\{-\left(\frac{2 \Omega_{1,2}}{\sigma_{1}^{2}\left(e^{\Omega_{1,2}(\tau-\xi)}-1\right)}\right) v_{1}-\left(\frac{2 \Omega_{2,2}}{\sigma_{2}^{2}\left(e^{\Omega_{2,2}(\tau-\xi)}-1\right)}\right) v_{2}\right\} e^{i \eta(z-\ln S)-i \eta(r-q)(\tau-\xi)} \\
\times & \left(\frac{2 \Omega_{1,2}}{\left(\Theta_{1,2}-i \eta \sigma_{1}^{2} b_{1}(\xi)-\Omega_{1,2}\right)\left(1-e^{-\Omega_{1,2}(\tau-\xi)}\right)+2 \Omega_{1,2}}\right)^{\frac{2 \Phi_{1}}{\sigma_{1}^{2}}} \\
\times & \left.\left(\frac{2 \Omega_{2,2}}{\left(\Theta_{2,2}-i \eta \sigma_{2}^{2} b_{2}(\xi)-\Omega_{2,2}\right)\left(1-e^{-\Omega_{2,2}(\tau-\xi)}\right)+2 \Omega_{2,2}}\right)^{\frac{2 \Phi_{2}^{2}}{\sigma_{2}^{2}}} \times \frac{2 \Omega_{1,2}}{\sigma_{1}^{2}\left(e^{\Omega_{1,2}(\tau-\xi)}-1\right)}\right\} \\
& \left.\times \exp \left\{\frac{2 \Omega_{1,2} v_{1}}{\left(\Theta_{1,2}-i \eta \sigma_{1}^{2} b_{1}(\xi)-\Omega_{1,2}\right)\left(1-e^{-\Omega_{1,2}(\tau-\xi)}\right)+2 \Omega_{1,2}} \times \frac{2 \Omega_{2,2} v_{2}}{\sigma_{2}^{2}\left(e^{\Omega_{2,2}(\tau-\xi)}-1\right)}\right\}\right] d \eta .
\end{aligned}
$$

Proceeding the way we handled equation (A8.14) the above equation simplifies to

$$
J_{2}(\xi, z)=\frac{1}{2 \pi} \int_{-\infty}^{\infty} e^{i \eta z} \hat{g}_{2}^{A}\left(\tau-\xi, S, v_{1}, v_{2} ;-\eta, b_{1}, b_{2}\right) d \eta
$$

where

$$
\begin{aligned}
& \hat{g}_{2}^{A}\left(\tau, S, v_{1}, v_{2} ; \eta, b_{1}, b_{2}\right)=\exp \left\{i \eta \ln S+B_{2}^{A}\left(\tau, \eta, b_{1}, b_{2}\right)+D_{1,2}^{A}\left(\tau, \eta, b_{1}\right) v_{1}+D_{2,2}^{A}\left(\tau, \eta, b_{2}\right) v_{2}\right\}, \\
& \begin{array}{r}
B_{2}^{A}(\tau, \eta)=i \eta(r-q) \tau+\frac{\Phi_{1}}{\sigma_{1}^{2}}\left\{\left(\Theta_{1,2}-\Omega_{1,2}\right) \tau-2 \ln \left(\frac{\left(\Theta_{1,2}+i \eta \sigma_{1}^{2} b_{1}-\Omega_{12}\right)\left(1-e^{-\Omega_{1,2} \tau}\right)+2 \Omega_{1,2}}{2 \Omega_{1,2}}\right)\right\} \\
\quad+\frac{\Phi_{2}}{\sigma_{2}^{2}}\left\{\left(\Theta_{2,2}-\Omega_{2,2}\right) \tau-2 \ln \left(\frac{\left(\Theta_{2,2}+i \eta \sigma_{2}^{2} b_{2}-\Omega_{2,2}\right)\left(1-e^{-\Omega_{2,2} \tau}\right)+2 \Omega_{2,2}}{2 \Omega_{2,2}}\right)\right\}, \\
D_{1,2}^{A}(\tau, \eta)=\frac{\Theta_{1,2}-\Omega_{1,2}}{\sigma_{1}^{2}}-\frac{2 \Omega_{1,2}}{\sigma_{1}^{2}\left(e^{\Omega_{1,2} \tau}-1\right)}+\frac{2 \Omega_{1,2}}{\left(\Theta_{1,2}+i \eta \sigma_{1}^{2} b_{1}-\Omega_{1,2}\right)\left(1-e^{-\Omega_{1,2} \tau}\right)+2 \Omega_{1,2}} \times \frac{2 \Omega_{1,2}}{\sigma_{1}^{2}\left(e^{\Omega_{1,2} \tau}-1\right)} \\
D_{2,2}^{A}(\tau, \eta)=\frac{\Theta_{2,2}-\Omega_{2,2}}{\sigma_{2}^{2}}-\frac{2 \Omega_{2,2}}{\sigma_{2}^{2}\left(e^{\Omega_{2,2} \tau}-1\right)}+\frac{2 \Omega_{2,2}}{\left(\Theta_{2,2}+i \eta \sigma_{2}^{2} b_{2}-\Omega_{2,2}\right)\left(1-e^{-\Omega_{2,2} \tau}\right)+2 \Omega_{2,2}} \times \frac{\sigma_{2}^{2}\left(e^{\Omega_{2,2} \tau}-1\right)}{(A 10,8)} .
\end{array}
\end{aligned}
$$

The functions $B_{2}^{A}(\tau, \eta), D_{1,2}^{A}(\tau, \eta)$ and $D_{2,2}^{A}(\tau, \eta)$ can be simplified further by letting

$$
Q_{1,2}^{A}=\frac{\Theta_{1,2}+i \eta \sigma_{1}^{2} b_{1}+\Omega_{1,2}}{\Theta_{1,2}+i \eta \sigma_{1}^{2} b_{1}-\Omega_{1,2}}, \quad \text { and } \quad Q_{2,2}^{A}=\frac{\Theta_{2,2}+i \eta \sigma_{2}^{2} b_{2}+\Omega_{2,2}}{\Theta_{2,2}+i \eta \sigma_{2}^{2} b_{2}-\Omega_{2,2}}
$$

such that 


$$
\begin{gathered}
B_{2}^{A}\left(\tau, \eta, b_{1}, b_{2}\right)=i \eta(r-q) \tau+\frac{\Phi_{1}}{\sigma_{1}^{2}}\left\{\left(\Theta_{1,2}+\Omega_{1,2}\right) \tau-2 \ln \left(\frac{1-Q_{1,2}^{A} e^{\Omega_{1,2} \tau}}{1-Q_{1,2}^{A}}\right)\right\} \\
+\frac{\Phi_{2}}{\sigma_{2}^{2}}\left\{\left(\Theta_{2,2}+\Omega_{2,2}\right) \tau-2 \ln \left(\frac{1-Q_{2,2}^{A} e^{\Omega_{2,2} \tau}}{1-Q_{2,2}^{A}}\right)\right\}, \\
D_{1,2}^{A}\left(\tau, \eta, b_{1}\right)=-i \eta b_{1}+\frac{\left(\Theta_{1,2}+\Omega_{1,2}\right)}{\sigma_{1}^{2}}\left[\frac{1-e^{\Omega_{1,2} \tau}}{1-Q_{1,2}^{A} e^{\Omega_{1,2} \tau}}\right], \\
D_{2,2}^{A}\left(\tau, \eta, b_{2}\right)=-i \eta b_{2}+\frac{\left(\Theta_{2,2}+\Omega_{2,2}\right)}{\sigma_{2}^{2}}\left[\frac{1-e^{\Omega_{2,2} \tau}}{1-Q_{2,2}^{A} e^{\Omega_{2,2} \tau}}\right] .
\end{gathered}
$$

By using a similar transformation to that between $Q_{1}$ and $Q_{2}$ in Appendix 7 we can write

$$
\hat{g}_{2}^{A}\left(\tau-\xi, S, v_{1}, v_{2} ; \eta-i, b_{1}, b_{2}\right)=e^{(r-q)(\tau-\xi)} S \hat{g}_{1}^{A}\left(\tau-\xi, S, v_{1}, v_{2} ; \eta, b_{1}, b_{2}\right),
$$

where

$$
\begin{aligned}
& \hat{g}_{1}^{A}\left(\tau, S, v_{1}, v_{2} ; \eta, b_{1}, b_{2}\right)=\exp \left\{i \eta \ln S+B_{1}^{A}\left(\tau, \eta, b_{1}, b_{2}\right)+D_{1,1}^{A}\left(\tau, \eta, b_{1}\right) v_{1}+D_{2,1}^{A}\left(\tau, \eta, b_{2}\right) v_{2}\right\} \\
& \begin{aligned}
B_{1}^{A}\left(\tau, \eta, b_{1}, b_{2}\right)=i \eta(r-q) \tau+\frac{\Phi_{1}}{\sigma_{1}^{2}}\left\{\left(\Theta_{1,1}+\Omega_{1,1}\right) \tau-2 \ln \left(\frac{1-Q_{1,1}^{A} e^{\Omega_{1,2} \tau}}{1-Q_{1,1}^{A}}\right)\right\} \\
+\frac{\Phi_{2}}{\sigma_{2}^{2}}\left\{\left(\Theta_{2,1}+\Omega_{2,1}\right) \tau-2 \ln \left(\frac{1-Q_{2,1}^{A} e^{\Omega_{2,1} \tau}}{1-Q_{2,1}^{A}}\right)\right\} \\
D_{1,1}^{A}\left(\tau, \eta, b_{1}\right)=-i \eta b_{1}+\frac{\left(\Theta_{1,1}+\Omega_{1,1}\right)}{\sigma_{1}^{2}}\left[\frac{1-e^{\Omega_{1,1} \tau}}{1-Q_{1,1}^{A} e^{\Omega_{1,1} \tau}}\right] \\
D_{2,1}^{A}\left(\tau, \eta, b_{2}\right)=-i \eta b_{2}+\frac{\left(\Theta_{2,1}+\Omega_{2,1}\right)}{\sigma_{2}^{2}}\left[\frac{1-e^{\Omega_{2,1} \tau}}{1-Q_{2,1}^{A} e^{\Omega_{2,1} \tau}}\right] .
\end{aligned}
\end{aligned}
$$

Incorporating these into equation (A10.3) we obtain

$$
\begin{gathered}
V_{P}^{A}\left(\tau, S, v_{1}, v_{2}\right)=\int_{0}^{\tau} e^{-r(\tau-\xi)} \int_{b_{0}(\xi)}^{\infty} q e^{z} \frac{1}{2 \pi} \int_{-\infty}^{\infty} e^{i \eta z} e^{(r-q)(\tau-\xi)} S \hat{g}_{1}^{A}\left(\tau-\xi, S, v_{1}, v_{2} ;-\eta, b_{1}(\xi), b_{2}(\xi)\right) d \eta d z d \xi \\
-\int_{0}^{\tau} e^{-r(\tau-\xi)} \int_{b_{0}(\xi)}^{\infty} r K \frac{1}{2 \pi} \int_{-\infty}^{\infty} e^{i \eta z} \hat{g}_{2}^{A}\left(\tau-\xi, S, v_{1}, v_{2} ;-\eta, b_{1}(\xi), b_{2}(\xi)\right) d \eta d z d \xi
\end{gathered}
$$

which implies that

$$
\begin{gathered}
V_{P}^{A}\left(\tau, S, v_{1}, v_{2}\right)=\int_{0}^{\tau} e^{-r(\tau-\xi)} q S e^{(r-q)(\tau-\xi)} \frac{1}{2 \pi} \int_{-\infty}^{\infty} \hat{g}_{1}^{A}\left(\tau-\xi, S, v_{1}, v_{2} ;-\eta, b_{1}(\xi), b_{2}(\xi)\right) \int_{b_{0}(\xi)}^{\infty} e^{z} e^{i \eta z} d z \eta d \xi \\
-\int_{0}^{\tau} e^{-r(\tau-\xi)} r K \frac{1}{2 \pi} \int_{-\infty}^{\infty} \hat{g}_{2}^{A}\left(\tau-\xi, S, v_{1}, v_{2} ;-\eta, b_{1}(\xi), b_{2}(\xi)\right) \int_{b_{0}(\xi)}^{\infty} e^{i \eta z} d z d \eta d \xi
\end{gathered}
$$


Proceeding as we did in equation (A8.18) we obtain the result that

$$
\begin{aligned}
V_{P}\left(\tau, S, v_{1}, v_{2}\right)= & \int_{0}^{\tau}\left[q e^{-q(\tau-\xi)} S \hat{P}_{1}^{A}\left(\tau-\xi, S, v_{1}, v_{2} ; b_{0}(\xi), b_{1}(\xi), b_{2}(\xi)\right)\right. \\
& \left.-r e^{-r(\tau-\xi)} K \hat{P}_{2}^{A}\left(\tau-\xi, S, v_{1}, v_{2} ; b_{0}(\xi), b_{1}(\xi), b_{2}(\xi)\right)\right] d \xi
\end{aligned}
$$

where

$\hat{P}_{j}^{A}\left(\tau-\xi, S, v_{1}, v_{2} ; b_{0}(\xi), b_{1}(\xi), b_{2}(\xi)\right)=\frac{1}{2}+\frac{1}{\pi} \int_{0}^{\infty} \operatorname{Re}\left(\frac{\hat{g}_{j}^{A}\left(\tau-\xi, S, v_{1}, v_{2} ; \eta, b_{1}(\xi), b_{2}(\xi)\right) e^{-i \eta b_{0}(\xi)}}{i \eta}\right) d \eta$

for $j=1,2$, which is the result presented in Proposition 11.1.

\section{References}

Abramowitz, M. and Stegun, I. A. (1964), 'Handbook of Mathematical Functions', Applied Mathematics Series, Dover, New York 55.

Adolfsson, T., Chiarella, C., Ziogas, A. and Ziveyi, J. (2009), 'Pricing American Options under Stochastic Volatility', Working Paper, School of Finance and Economics, University of Technology, Sydney .

Ait-Sahlia, F. and Lai, T. L. (2001), 'Exercise Boundaries and Efficient Approximations to American Option Prices and Hedge Parameters', Journal of Computational Finance 4(4), 85-96.

Albrecher, H., Mayer, P., Schoutens, W. and Tistaert, J. (2007), ' The Little Heston Trap', Wilmott Magazine January, 83-92.

Black, F. and Scholes, M. (1973), 'The Pricing of Corporate Liabilities', Journal of Political Economy 81, 637-659.

Blattberg, R. C. and Gonedes, N. J. (1974), ' A comparison of the Stable and Student Distributions as Statistical Models for Stock Prices', Journal of Business 47(2), 244-280.

Broadie, M., Detemple, J., Ghysels, E. and Torres, O. (2000), 'American Options with Stochastic Dividends and Volatility: A Nonparametric Investigation', Journal of Econometrics 94, 53-92.

Cheang, G., Chiarella, C. and Ziogas, A. (2009), ' The Representation of American Option Prices Under Stochastic Volatility and Jump Diffusion Dynamics', Quantitative Finance Research Centre, University of Technology Sydney Research Paper No: 256.

Chiarella, C. (2010), 'An Introduction to Derivative Security Pricing: Techniques, Methods and Applications', Springer, Forthcoming .

Chiarella, C., El-Hassan, N. and Kucera, A. (1999), ' Evaluation of American option prices in a path integral framework using Fourier-Hermite series expansion', Journal of Economic Dynamics and Control 23, 1387-1424.

Chiarella, C., Kang, B., Meyer, G. H. and Ziogas, A. (2009), ' The Evaluation of American Option prices Under Stochastic Volatility and Jump-Diffusion Dynamics Using the Method of Lines', International Journal of Theoretical and Applied Finance 12(3), 393-425.

Clark, P. K. (1973), 'A Subordinated Stochastic Process Model with Finite Variance for Speculative Prices', Econometrica 41(1), 135-155.

Clarke, N. and Parrott, K. (1999), 'Multigrid for American Option Pricing with Stochastic Volatility', Applied Mathematical Finance 6(3), 177-195.

da Fonseca, J., Grasselli, M. and Tebaldi, C. (2005), 'Wishart Multi-Dimensional Stochastic Volatility', Working paper ESILV, RR-31. 
da Fonseca, J., Grasselli, M. and Tebaldi, C. (2008), ' A multifactor Volatility Heston Model', Quantitative Finance 8(6), 591-604.

Detemple, J. and Tian, W. (2002), 'The Valuation of American Options for a Class of Diffusion Processes', Management Science 48(7), 917-937.

Feller, W. (1951), 'Two Singular Diffusion Problems', The Annals of Mathematics 54(1), 173-182.

Harrison, J. M. (1990), ' Brownian Motion and Stochastic Flow Systems ', Krieger Publishing Company, Malabar, Florida.

Heston, S. L. (1993), 'A Closed-Form Solution for Options with Stochastic Volatility with Applications to Bonds and Currency Options', The Review of Financial Studies 6(2), 327-343.

Huang, J., Subrahmanyam, M. G. and Yu, G. G. (1996), 'Pricing and Hedging American Options: A Recursive Integration Method', Review of Financial Studies 9, 277-300.

Hull, J. and White, A. (1987), ' The Pricing of Options on Assets with Stochastic Volatility', Journal of Finance XLII(2), 281-300.

Ikonen, S. and Toivanen, J. (2004), 'Operator Splitting Methods for American Options with Stochastic Volatility', Applied Mathematics Letters 17, 809-814.

Jamshidian, F. (1992), 'An Analysis of American Options', Review of Futures Markets 11, 72-80.

Ju, N. (1998), 'Pricing an American Option by Approximating its Early Exercise Boundary as a Multipiece Exponential Function', Review of Financial Markets 11, 627-646.

Kahl, C. and Jäckel, P. (2005), 'Not-So-Complex Logarithms in the Heston Model', Wilmott Magazine September.

Kallast, S. and Kivinukk, A. (2003), 'Pricing and Hedging American Options using Approximations by Kim Integral Equations', European Finance Review 7, 361-383.

Karatzas, I. (1988), 'On the Pricing of American Option', Applied Mathematics and Optimization 17, 37-60.

Kim, I. J. (1990), 'The Analytical Valuation of American Options', Review of Financial Studies 3, $547-572$.

Latané, H. A. and Rendleman, R. J. (1976), 'Standard Deviations of Stock Price Ratios Implied in Option Prices', The Journal of Finance XXXI(2), 369-381.

Logan, J. D. (2004), 'Applied Partial Differential Equations', Springer .

Mallier, R. (2002), 'Evaluating Approximations to the Optimal Exercise Boundary for American Options', Journal of Applied Mathematics 2, 71-92.

Mandelbrot, B. (1963), 'The Variation of Certain Speculative Prices', The Journal of Business 36, 394419.

Officer, R. R. (1972), 'The Distribution of Stock Returns', Journal of the American Statistical Association 67(340), 807-812.

Platen, E. and Rendek, R. (2008), ' Empirical Evidence on Student- $t$ Log-Returns of Diversified World Stock Indicies', Journal of Statistical Theory and Practice 2(2), 233-251.

Rosenberg, B. (1972), 'The Behavior of Random Variables with Nonstationary Variance and the Distribution of Security Prices', Stochastic Volatility: Selected Readings by Neil Shephard, Oxford University Press 2005 Edition pp. 83-103.

Scott, L. O. (1987), 'Option Pricing when the Variance Changes Randomly: Theory, Estimation and Application', Journal of Financial and Quantitative Analysis 22(4), 419-438.

Shephard, N. G. (1991), 'From Characteristic Function to Distribution Function: A Simple Framework for the Theory', Econometric Theory 7, 519-529.

Stein, E. M. and Stein, J. C. (1991), 'Stock Price Distributions with Stochastic Volatility: An Analytical Approach', The Review of Financial Studies 4(4), 727-752.

Touzi, N. (1999), 'American Options Exercise Boundary when the Volatility Changes Randomly', Applied Mathematics and Optimization 39, 411-422. 
Tzavalis, E. and Wang, S. (2003), 'Pricing American Options under Stochastic Volatility: A New Method using Chebyshev Polynomials to Approximate the Early Exercise Boundary', Queen Mary, University of London, School of Economics and Finance Working Paper No: 488.

Wiggins, J. B. (1987), 'Option Values under Stochastic Volatility: Theory and Empirical Estimates', Journal of Financial Economics 19, 351-372. 